\title{
Isoperimetric profiles and random walks on some permutation wreath products
}

\author{
Laurent Saloff-Coste* \\ Department of Mathematics \\ Cornell University
}

\author{
Tianyi Zheng \\ Department of Mathematics \\ Stanford University
}

\begin{abstract}
We study the isoperimetric profiles of certain families of finitely generated groups defined via marked Schreier graphs and permutation wreath products. The groups we study are among the "simplest" examples within a much larger class of groups, all defined via marked Schreier graphs and/or action on rooted trees, which includes such examples as the long range group, Grigorchuck group and the basillica group. The highly nonlinear structure of these groups make them both interesting and difficult to study. Because of the relative simplicity of the Schreier graphs that define the groups we study here (the key fact is that they contained very large regions that are "one dimensional"), we are able to obtain sharp explicit bounds on the $L^{1}$ and $L^{2}$ isoperimetric profiles of these groups. As usual, these sharp isoperimetric profile estimates provide sharp bounds on the probability of return of simple random walk. Nevertheless, within each of the families of groups we study there are also many cases for which the existing techniques appear inadequate and this leads to a variety of open problems.
\end{abstract}

\section{Introduction}

In the study of random walks on groups, some of the most basic and compelling questions are to understand what structural properties of a group impact the behavior of random walk and how this impact can be captured precisely. Naturally, this also leads to the question of describing what random walk behaviors can possibly occur.

To any finitely generated group, one can associate the monotone non-increasing functions

$$
\Lambda_{1, G}, \Lambda_{2, G} \text { and } \Phi_{G}
$$

which, respectively, describe the $L^{1}$ - and $L^{2}$-isoperimetric profiles and the return probability (or heat kernel decay) associated with the group $G$ (precise

\footnotetext{
*Partially supported by NSF grant DMS 1004771 and DMS 1404435
} 
definitions are recalled below in Section 2.4). From a coarse point of view explained in Section 2.4. these are group invariants in the sense that they do not depend on the particular choice of symmetric finite generating set that is used to define them. A celebrated results of Følner and Kesten asserts that the dichotomy between amenable and non-amenable groups can be captured precisely using any one of these three invariants: A group is amenable if and only if $\Lambda_{1, G}$ (equivalently, $\Lambda_{2, G}$ ) is bounded below away from 0 , and this is also equivalent to having $\Phi_{G}$ decay exponentially fast.

This paper focuses on these invariants and how they depend on the structure of the underlying group in the context of several interesting family of amenable groups. Let us stress that there are other related random walk characteristics such as entropy and speed that are of great interest but are not discussed here.

To put this work in perspective, recall that among polycyclic groups or (almost equivalently) finitely generated discrete amenable subgroups of linear groups, the behaviors of $\Lambda_{1, G}, \Lambda_{2, G}$ and $\Phi_{G}$ are well understood and fall in exactly 2 possible categories:

- The group $G$ has exponential volume growth and

$$
\Lambda_{1, G}(v)^{2} \simeq \Lambda_{2, G} \simeq \frac{1}{[\log (1+v)]^{2}} \text { and } \Phi_{G}(n) \simeq \exp \left(-n^{1 / 3}\right) .
$$

- The volume growth $V_{G}$ satisfies $V_{G}(r) \simeq(1+r)^{d}$ for some integer $d$ and

$$
\Lambda_{1, G}(v)^{2} \simeq \Lambda_{2, G}(v) \simeq(1+v)^{-2 / d} \text { and } \Phi_{G}(n) \simeq(1+n)^{-d / 2} .
$$

These can be considered as the "classical" behaviors. See 31 for the description of a larger class of groups for which only these behaviors can occur.

By now it is well-known that, for more general groups, other behaviors can occur. See [11, 24, 26]. For instance, the authors show in 28, that the free solvable group $\mathbf{S}_{d, r}$ of derived length $d>2$ on $r$ generators satisfies

$$
\Lambda_{1, \mathbf{S}_{d, r}}(v)^{2} \simeq \Lambda_{2, \mathbf{S}_{d, r}}(v) \simeq\left(\frac{\log _{d}(v)}{\log _{d-1}(v)}\right)^{2 / r}
$$

and

$$
\Phi_{\mathbf{S}_{d, r}}(n) \simeq \exp \left(-n\left(\frac{\log _{d-1}(n)}{\log _{d-2}(n)}\right)^{2 / r}\right) .
$$

Here, $\log _{d}$ denotes the iterated logarithm, $d$-times. See 28] for the statement when $d=2$ in which case the formula for $\Phi_{\mathbf{S}_{d, r}}$ must be modified (the estimates for $\Lambda_{1, \mathbf{S}_{d, r}}$ and $\Lambda_{2, \mathbf{S}_{d, r}}$ remain valid as stated above).

Following the groundbreaking work of R. Grigorchuck regarding groups of intermediate volume growth and the many works that followed, it has become apparent that it is important to consider the case of subgroups of the automorphism group of a rooted tree as well as groups defined via explicit marked 
Schreier graphs (the word "explicit" is important here as any finitely generated group can be "defined" by its action on one of its marked Cayley graph).

In this paper we study a collections of examples of such groups and their associated permutation wreath products. Most of the paper is devoted to two families. The first family has been considered in 21] where they are called "bubble groups" (see Section [5). We call the other family "cyclic NeumannSegal groups" (see Section 6). It is part of a larger family considered by a number of different authors after some interesting properties were pointed out in [23. We obtain estimates on the $L^{1}$ - and $L^{2}$-isoperimetric profiles of groups in these families. As is well-known, this yields assorted results for the probability of return of associated random walks. The examples we consider are, in a sense, among the simplest in the very large family alluded to above. The key feature that distinguishes these examples is that the Schreier graphs that are used to defined them have large one-dimensional pieces at all scales. This allow us to capture the isoperimetric profiles of the wreath extensions of some of these groups in a rather precise way. The resulting observed behaviors are diverse and quite interesting.

For example, we study a family of groups depending on a continuous parameter $\gamma>1$ and for which we show that

$$
\Phi_{G}(n) \simeq \exp \left(-n 2^{-2\left((1+\gamma) \log _{2}(n)\right)^{\gamma /(1+\gamma)}}\right) .
$$

See Remark 6.17 (the coarse equivalence $\simeq$ is defined in 2.4). We also obtain examples with

$$
\Phi_{G}(n) \simeq \exp \left(-n^{\gamma}(\log n)^{1-\gamma}\right)
$$

for each $\gamma \in[1 / 3,1)$ (see Example 5.11 with $\gamma=\frac{\beta+1}{3 \beta+1}, \beta \in(0, \infty)$ ) and

$$
\Phi_{G}(n) \simeq \exp \left(-n^{1 / 3}(\log n)^{2(1+\kappa) / 3}\right)
$$

for each $\kappa>0$ (see Example 5.12). We discuss a family of groups such that, for any $\gamma \in(1 / 3,1)$, there is a group $G$ in the family for which

$$
\exp \left(-n^{\frac{\gamma+1}{3-\gamma}}(\log n)^{\frac{2(1-\gamma)}{3-\gamma}}\right) \lesssim \Phi_{G}(n) \lesssim \exp \left(-n^{\gamma}(\log n)^{1-\gamma}\right)
$$

with both extremes attained at certain times and a detailed coarse description of $\Phi_{G}$ available at all times. See 6.18 with $\gamma=\varkappa /(3 \varkappa-2), \varkappa \in(1, \infty)$. These examples demonstrate the existence of a continuum of distinct (and explicit) behaviors and, for each, we obtain corresponding estimates for the functions $\Lambda_{1, G}$ and $\Lambda_{2, G}$.

The groups we study provided a host of additional behaviors. In particular, they provides examples of pairs of groups $G_{1}, G_{2}$ for which the behaviors of the functions $\Lambda_{1, G_{i}}, \Lambda_{2, G_{i}}$ and $\Phi_{G_{i}}$ can be described explicitly and such that the functions $\Lambda_{1, G_{1}}$ and $\Lambda_{1, G_{2}}$ are not comparable in the sense that

$$
\liminf _{n \rightarrow \infty} \frac{\Lambda_{1, G_{1}}}{\Lambda_{1, G_{2}}}=0 \text { and } \limsup _{n \rightarrow \infty} \frac{\Lambda_{1, G_{1}}}{\Lambda_{1, G_{2}}}=\infty
$$


with similar statement holding as well for $\Lambda_{2, G_{i}}$ and $\Phi_{G_{i}}, i=1,2$. Explicitly, fix $\gamma \in(1 / 3,1)$ and pick $G_{1}$ so that 1.1 holds. Pick $\gamma^{\prime} \in(1 / 3, \gamma)$ so that

$$
\gamma^{\prime}<\gamma<\frac{\gamma^{\prime}+1}{3-\gamma^{\prime}}=\gamma^{\prime}+\frac{\left(\gamma^{\prime}-1\right)^{2}}{3-\gamma^{\prime}}
$$

(for instance $\gamma^{\prime}=2 / 5<\gamma=1 / 2<7 / 13$ ) and let $G_{2}$ be such that (1.2) holds with parameter $\gamma^{\prime}$. The various family of groups we study offer many further opportunities, which we do not pursue explicitly, to construct such examples.

The families we consider are rich enough that many intriguing questions remain open. For one thing, most of our sharp results concern wreath extensions $\mathbb{Z} \imath_{\mathcal{S}} \Gamma$ where $\Gamma$ is defined by its action on a mark Schreier graph $\mathcal{S}$ and, for the most part, understanding the isoperimetric profile of $\Gamma$ itself is an open question. Also, the groups in the two main families we consider depend on the choice of one (or two) infinite sequence(s) of integers and our analysis provides sharp results only in a certain parameter range. This leaves much space for further studies.

This article is motivated by the works of a number of authors to whom we have borrowed results, problems and ideas. We make fundamental use of the work of A. Erschler on wreath products and adapt some of the ideas developed by K. Juschenko and her co-authors [18, 19, 20. The examples we consider (or some related cousins) have been studied before (with somewhat different viewpoints and focuses) in works including [3, 6, 13, 15, 16, 23, 30.

This article is organized as follows. Section 2 introduces definition and notation regarding group actions, Schreier graphs, groups of automorphisms of rooted trees and random walks. It contains the definitions of the $L^{1}$ - and $L^{2}$ isoperimetric profiles and that of the random walk invariant $\Phi_{G}$. Section 3 provide techniques that produce lower bounds on $\Lambda_{1, G}$ and $\Lambda_{2, G}$. We make significant use of Erschler's wreath product isoperimetric inequality and of related ideas developed in [27. By known techniques (e.g., 8] and [27, Section 2.1]), these translate into upper bounds on $\Phi_{G}$.

Section 4 develops abstract considerations with the goal of providing upper bounds on the $L^{2}$-isoperimetric profiles and assorted lower bound for $\Phi_{G}$. Regarding the $L^{1}$ - and $L^{2}$-isoperimetric profiles, in all the cases where we obtain sharp bounds, it turns out that $\Lambda_{1, G}^{2} \simeq \Lambda_{2, G}$ (whether or not this is true in general is a well-known and important open question). Since we always have $\Lambda_{1, G}^{2} \lesssim \Lambda_{2, G}$, proving that $\Lambda \lesssim \Lambda_{1, G}$ and $\Lambda_{2, G} \lesssim \Lambda^{2}$ for some function $\Lambda$ is sufficient to prove that $\Lambda_{1, G}^{2} \simeq \Lambda_{2, G} \simeq \Lambda^{2}$.

Section 5 introduces the "bubble group" family. This is an uncountable family of groups parametrized by two integers sequences $\left(\mathbf{a}=\left(a_{1}, a_{2}, \ldots\right)\right.$ and $\mathbf{b}=\left(b_{1}, b_{2}, \ldots\right)$. We show that most of these groups have exponential volume growth (Lemma 5.1) and that they are amenable as long as $a_{n}$ tends to infinity (Proposition 5.13). The result of Section 3 apply readily but works is required to show how the abstract results of Section 4 applies to these examples. When $\mathbf{b}$ is constant $\left(b_{i}=b>2\right)$, and $a_{n}$ is increasing fast enough, we obtain matching two-sided bounds on $\Lambda_{1, G}, \Lambda_{2, G}$ and $\Phi_{G}$ where $G=\mathbb{Z} \imath_{\mathcal{S}} \Gamma_{\mathbf{a}, \mathbf{b}}$. Here $\Gamma_{\mathbf{a}, \mathbf{b}}$ is 
the bubble group associated with $\mathbf{a}, \mathbf{b}$ and $\mathcal{S}$ is its defining Schreier graph. See Theorem 5.8.

Section 6 is devoted to a sub-family (cyclic Neumann-Segal groups) of the family of Neumann-Segal groups. The cyclic Neumann-Segal groups are parametrized by a sequence of even integers. To study the isoperimetric profile of these groups and their wreath extensions, we apply the results of Section 3 (again, this is straightforward), and apply the result and ideas of Section 4. In one particular case of interest, we are able to obtain sharp results not only not only for the wreath extension $\mathbb{Z} \imath_{\mathcal{S}} \Gamma$ but also for the cyclic Neumann-Segal group $\Gamma$ itself. In fact, in this particular case, the groups $\Gamma$ and its wreath extension $\mathbb{Z} \imath_{\mathcal{S}} \Gamma$ have essentially the same behavior. See Theorem 6.14 and Remark 6.17

\section{Preliminaries}

\subsection{Group actions}

A left action of a group $\Gamma$ on a space $X$ is a map $\varphi_{L}: \Gamma \times X \rightarrow X$ such that

$$
\varphi_{L}\left(e_{\Gamma}, x\right)=x \text { and } \varphi_{L}\left(g_{1} g_{2}, x\right)=\varphi_{L}\left(g_{1}, \varphi_{L}\left(g_{2}, x\right)\right) .
$$

Similarly a right action of $\Gamma$ on $X$ is a map $\varphi_{R}: X \times \Gamma \rightarrow X$ such that

$$
\varphi_{R}\left(x, e_{\Gamma}\right)=x \text { and } \varphi_{R}\left(x, g_{1} g_{2}\right)=\varphi_{R}\left(\varphi_{R}\left(x, g_{1}\right), g_{2}\right) \text {. }
$$

In this article, it is natural to consider examples of both left and right actions, depending of the context. Given an action $\varphi_{*}$ of $\Gamma$ on $X$ where $*$ is either $L$ or $R$, we set

$$
g \cdot x= \begin{cases}\varphi_{L}(g, x) & \text { if } *=L \\ \varphi_{R}\left(x, g^{-1}\right) & \text { if } *=R .\end{cases}
$$

Let

$$
\mathcal{P}_{f}(X)=\oplus_{X} \mathbb{Z}_{2}
$$

be the set of all finite subsets of $X$. Any action of $\Gamma$ on $X$ extends to an action of $\Gamma$ on $\mathcal{P}_{f}(X)$. If $f: X \rightarrow X$ is a function on $X$, and $g \in \Gamma$, we let $g \cdot f: X \rightarrow X$ be defined by

$$
g \cdot f(x)=f\left(g^{-1} \cdot x\right) .
$$

It follows that $\operatorname{supp}(g \cdot f)=g \cdot \operatorname{supp}(f)$.

Note that it is not rare that a group is, in fact, described by its action on a space $X$ (i.e., as a permutation group). We will encounter many such examples.

Example 2.1 (The finite dihedral groups $D_{2 n}$ ). The following two figures define the dihedral group $D_{2 n}$ of order $2 n=20$ generated by two elements of order 2 , $s$ and $t$, with $(s t)^{n}=e$. Figure 1 shows a marked Schreier graph on which the action of the group is faithful. It identifies the dihedral group as a particular subgroup of the symmetric group $S_{10}$ where the 10 objects that are permuted are the the vertices of the pictured line graph and the action of the generator $s$ 
and $t$ are described by the marked edges. In this particular case, since $s$ and $t$ are involutions, we do not need to indicate the edge orientation.

Figure 2 gives the marked Cayley graph of the same group with the same generators $s, t$ and with the identity element $e$ identified.

Figure 1: Marked Schreier graph

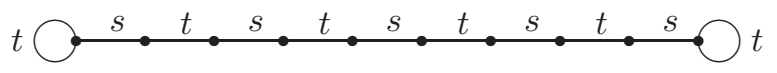

Figure 2: Marked Cayley graph

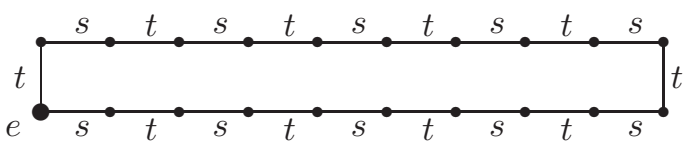

Figure 3: A function $f$ and the function $(t s) \cdot f$

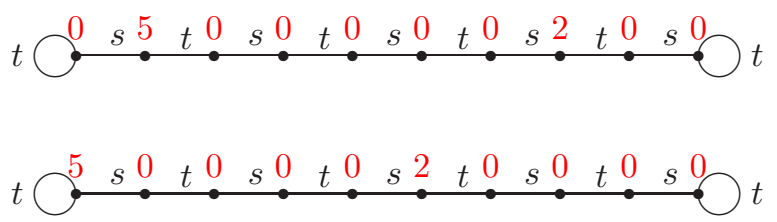

To understand how Picture 1 defines a subgroup of $\mathbb{S}_{10}$, imagine the vertex of the graph as fixed boxes which contains the labels $\{1, \ldots, 10\}$. The marking of the edges indicates how the group elements $s$ and $t$ each move the labels contained in the boxes, producing a permutation of the labels. A function $f$ on the Schreier graph is really a function on the labels $\{1, \ldots, 10\}$ and it can be pictured by indicating the value of $f(x)$ above $x$ (where label $x$ is in box $x$ ). Suppose $f=\mathbf{1}_{x_{0}}$ and $g \in \Gamma$. Then the function $g \cdot f$ is equal to $(g \cdot f)(x)=\mathbf{1}_{x_{0}}\left(g^{-1} \cdot x\right)=\mathbf{1}_{g \cdot x_{0}}(x)$. In words, to write down the picture describing the function $g \cdot f$, move the indicated values of $f$ along the Schreier graph according to the action of $g$.

\subsection{Permutational wreath product}

Let $\Gamma$ be a finitely generated group acting on a space $X$. Take a reference point $o$ in $X$ and let $\mathcal{S}$ be the orbital Schreier graph of $o$ under action of $\Gamma$. Given an auxiliary (finite or countable) group $H$, the permutational wreath product $H i_{\mathcal{S}} \Gamma$ is the semidirect product

$$
H r_{\mathcal{S}} \Gamma=\left(\oplus_{\mathcal{S}}(H)_{x}\right) \rtimes \Gamma
$$


where $\Gamma$ acts on the direct sum by permuting coordinates according to the action $(g, x) \mapsto g \cdot x$ of $\Gamma$ on $\mathcal{S}$. More precisely, the multiplication rule is given by

$$
(f, g)\left(f^{\prime}, g^{\prime}\right)=\left(f\left[g \cdot f^{\prime}\right], g g^{\prime}\right),
$$

where $f, f^{\prime}$ are functions $\mathcal{S} \rightarrow H$ with finite support, $g, g^{\prime} \in G$, and $\left(g \cdot f^{\prime}\right)(x)=$ $f^{\prime}\left(g^{-1} \cdot x\right)$ as defined earlier. Note that on the left-hand side of the equation displayed above, $(f, g)\left(f^{\prime}, g^{\prime}\right)$ indicates multiplication in $H z_{\mathcal{S}} \Gamma$ and that on the right-hand side, $f\left[g \cdot f^{\prime}\right]$ indicates multiplication of $f$ by $g \cdot f^{\prime}$ in $\oplus_{\mathcal{S}}(H)_{x}$ whereas $g g^{\prime}$ indicates multiplication in $\Gamma$.

Figure 4: Two elements $(f, g)$ and $\left(f^{\prime}, g^{\prime}\right)$ of $\mathbb{Z}_{2} \iota_{\mathcal{S}} \mathbb{D}_{20}$ and their product

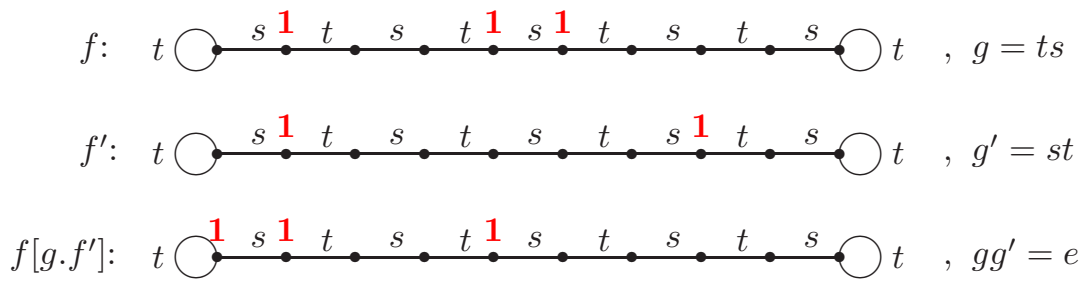

The ordinary wreath product with "lamp group" $H$ and "base group" $\Gamma$ corresponds to the case when $\Gamma$ acts on its own Cayley graph by left multiplication. We write $H \imath \Gamma$ for this ordinary wreath product.

The groups $H$ and $\Gamma$ are naturally embedded in $H \imath_{\mathcal{S}} \Gamma$ via the injective maps

$$
h \mapsto\left(\mathbf{1}_{h}^{o}, e_{\Gamma}\right), \text { where } \mathbf{1}_{h}^{o}(o)=h, \mathbf{1}_{k}^{o}(x)=e_{H} \text { if } x \neq o,
$$

and

$$
\gamma \mapsto\left(\mathbf{e}_{H}^{\mathcal{S}}, \gamma\right), \text { where } \mathbf{e}_{H}^{\mathcal{S}}(x)=e_{H} \text { for every } x \in \mathcal{S} .
$$

Let $\mu_{H}$ and $\mu_{\Gamma}$ be symmetric probability measures on $H$ and $\Gamma$ respectively. Using the above embeddings, $\mu_{H}$ and $\mu_{\Gamma}$ can be viewed as probability measures on $H \imath_{\mathcal{S}} \Gamma$ and we will often make this identification. The measure

$$
q=\mu_{H} * \mu_{\Gamma} * \mu_{H}
$$

on $H i_{\mathcal{S}} \Gamma$ is often referred to as the switch-walk-switch random walk where as

$$
\mathfrak{q}=\frac{1}{2}\left(\mu_{H}+\mu_{\Gamma}\right)
$$

is known as the the switch-or-walk measure on $H r_{\mathcal{S}} \Gamma$.

The random walk driven by $q$ on $H l_{\mathcal{S}} \Gamma$ shares some similarities with the switch-walk-switch random walk on ordinary wreath products. But there are some important differences. On the ordinary wreath product, if we consider random walk on the right, that is $\left\{X_{1} X_{2} \ldots X_{n}\right\}_{n}$ where $X_{i}$ are i.i.d. random 
variables distributed as $\mathfrak{q}$, the random walk can be understood in terms of a walker on the Cayley graph of $\Gamma$ who changes the lamp-configuration along its path. On the permutation wreath product $H i_{\mathcal{S}} \Gamma$ based on the Schreier graph $\mathcal{S}$, the switches happen along the inverted orbit. This creates much difficulty in analyzing the behavior of such random walks in the general case.

In what follows, we are going to consider random walk on the left, that is $S_{n}=X_{n} X_{n-1} \ldots X_{1}$ and much attention will be paid to translation of the support of the lamp configuration, rather than the inverted orbits directly. We will mostly work with the measure $\mathfrak{q}$.

\subsection{Groups acting on trees, activity}

Let $\bar{d}=\left(d_{j}\right)_{j \geq 1}$ be a sequence of integers $d_{j} \geq 2$. The spherical homogeneous rooted tree $\mathbb{T}_{\bar{d}}$ is the tree where each vertex at level $j$ has $d_{j+1}$ children in level $j+1$. The tree has a root at level 0 , which is denoted by the empty sequence $\emptyset$. A vertex at level $k$ is encoded by word $v=x_{1} \ldots x_{k-1} x_{k}$, where $x_{i}$ is a letter in the alphabet $\left\{0, \ldots, d_{i}-1\right\}$. Here a word is read from left to right. The integer $k$ is called the depth or level of the vertex $v$. We set $|v|=k$ and let $\mathbb{T}_{\bar{d}}^{k}$ denote the set of vertices in level $k$ of the tree $\mathbb{T}_{\bar{d}}$.

The group Aut $\left(\mathbb{T}_{\bar{d}}\right)$ is the group of automorphisms of $\mathbb{T}_{\bar{d}}$ fixing the root $\emptyset$. It is uncountable and is often equipped with the topology of convergence on finite sets which turns it into a locally compact group. For $v \in \mathbb{T}_{\bar{d}}$, consider the subtree $\mathbb{T}_{v, \bar{d}}$ of $\mathbb{T}_{\bar{d}}$ rooted at $v$. If $v$ is at level $k$, then this subtree is isomorphic to the spherical homogeneous rooted tree $\mathbb{T}_{\tau^{k}} \bar{d}$, where $\tau$ denotes the shift operator

$$
\tau\left(m_{1}, m_{2}, \ldots\right)=\left(m_{2}, m_{3}, \ldots\right) .
$$

The automorphism group $\operatorname{Aut}\left(\mathbb{T}_{\bar{d}}\right)$ admits the following canonical description called "wreath recursion",

$$
\begin{gathered}
\operatorname{Aut}\left(\mathbb{T}_{\bar{d}}\right) \simeq \operatorname{Aut}\left(\mathbb{T}_{\tau \bar{d}}\right){\chi_{\mathbb{T}}^{1}}_{\bar{d}} S_{d_{1}}, \\
g \mapsto\left(g_{0}, \ldots, g_{d_{1}-1}\right) \sigma .
\end{gathered}
$$

Each $g_{i}$ is called the section (or restriction) of $g$ at vertex $i$, it gives the action of $g$ on the subtree rooted at $i$. The rooted component $\sigma$ describes how these subtrees are permuted. Given a word $v=x_{1} \ldots x_{k}$, the right action is defined recursively by

$$
\varphi_{R}\left(x_{1} \ldots x_{k}, g\right)=\sigma\left(x_{1}\right) \varphi_{R}\left(x_{2} \ldots x_{k}, g_{x_{1}}\right) .
$$

One can also write the wreath recursion at level $k$ to have a canonical isomorphism

$$
\begin{aligned}
\operatorname{Aut}\left(\mathbb{T}_{\bar{d}}\right) & \simeq \operatorname{Aut}\left(\mathbb{T}_{\tau^{k} \bar{d}}\right){l_{\mathbb{T}}}_{\bar{d}} \operatorname{Aut}\left(\mathbb{T}_{\bar{d}}^{k}\right), \\
g & \mapsto\left(g_{v}^{k}, v \in \mathbb{T}_{\bar{d}}^{k}\right) \sigma^{k} .
\end{aligned}
$$

where the subscript $k$ indicates the level (not a power). In most case, the subscript $k$ is omitted because it is clear from the context that the decomposition 
is done at level $k$. Each $g_{v}=g_{v}^{k}$ is called the section (or restriction) of $g$ at vertex $v$, it describes the action of $g$ on the subtree rooted at $v$. The permutation $\sigma=\sigma^{k}$ describes how vertices on level $k$ are permuted.

Since the isomorphisms are canonical, we identify $g$ with its image under the wreath recursions and, given a level $k$, write

$$
g=\left(g_{v}, v \in \mathbb{T}_{\bar{d}}^{k}\right) \sigma .
$$

For every $g \in \operatorname{Aut}\left(\mathbb{T}_{\bar{d}}\right)$ the activity function $a_{g}(k)$ defined in [30, Section 2.4] counts the number of nontrivial sections $g_{v}$ at each level $k$, that is

$$
a_{g}(k)=\#\left\{v \in \mathbb{T}_{\bar{d}}^{k}: g_{v} \neq e\right\} .
$$

We say $G<\operatorname{Aut}\left(\mathbb{T}_{\bar{d}}\right)$, is a group of bounded activity if for every element $g \in G, \sup _{k} a_{g}(k)<\infty$. When $G$ is finitely generated, it's sufficient to check for each generator of $G$ if it's activity growth is bounded.

Example 2.2. Figures 57 describe (a) the Cayley graph of the infinite dihedral group $D_{\infty}=<s, t: s^{2}=t^{2}=e>$, (b) a marked Schreier graph that can be used to define $D_{\infty}$, and (c) the generator $t$ as an element of the automorphism group of the rooted binary tree $\operatorname{Aut}\left(\mathbb{T}_{\overline{2}}\right)$. Here we set $s\left(x_{1} x_{2} \ldots x_{k}\right)=\overline{x_{1}} x_{2} \ldots x_{k}$ with $\overline{x_{1}}=x_{1}+1 \bmod 2$, that is $s=(e, e) \tau$ where $\tau$ is the transposition at level 1 , and $t=(t, s) 1$ where 1 stands the identity permutation at level 1 . Obviously, the definition of $t$ is recursive. If $x=x_{1} \ldots x_{k}$ with $j$ being the first index such that $x_{j}=1$ then $t(x)=x_{1} \ldots x_{j} \overline{x_{j+1}}, \ldots, x_{k}$. The activity $a_{s}(k)$ vanishes for $k>1$. The activity $a_{t}(k)=1$ for all $k$. The very notable difference between $s$ and $t$ viewed as automorphisms of the tree is an artifact of this representation.

Note that $t$ leaves invariant the end $o=0^{\infty}$ of the tree which corresponds to the left most vertex in the Schreier graph depicted on Figure 6. In order to understand Figure 6 in terms of the binary tree and Figure 7 one simply consider the orbit of $o=0^{\infty}$ under $D_{\infty}=<s, t>\subset \operatorname{Aut}\left(\mathbb{T}_{\overline{2}}\right)$.

Figure 5: Marked Cayley graph for $D_{\infty}=<s, t: s^{2}=t^{2}=e>$

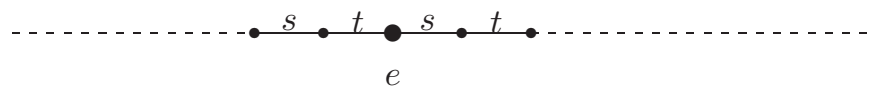

Figure 6: Marked Schreier graph for $D_{\infty}$

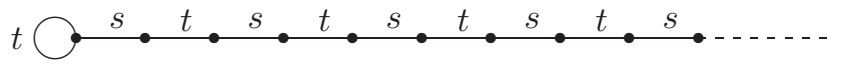


Figure 7: The generator $t$ viewed as an element of $\operatorname{Aut}\left(\mathbb{T}_{\overline{2}}\right)$

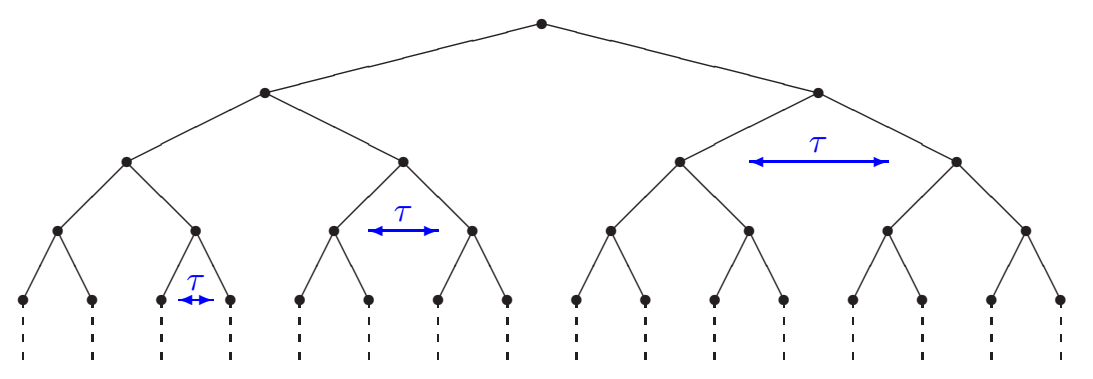

The stabilizers and rigid stabilizers are very important in the study of branch groups ([15, 16]). Let $\Gamma$ be a subgroup of $\operatorname{Aut}\left(\mathbb{T}_{\bar{d}}\right)$. Given a vertex $u \in \mathbb{T}_{\bar{d}}$, the stabilizer of $u$ in $\Gamma$ is the subgroup

$$
\operatorname{St}_{\Gamma}(u)=\{g \in \Gamma: g \cdot u=u\}
$$

of $\mathbb{T}_{\bar{d}}$. The subgroup

$$
\operatorname{St}_{\Gamma}(k)=\cap_{u \in \mathbb{T}_{\bar{d}}} \operatorname{St}_{\Gamma}(u)
$$

is called the level $k$ stabilizer. The rigid vertex stabilizer is

$$
\operatorname{rist}_{\Gamma}(u)=\left\{g \in \Gamma: g \cdot v=v \text { for all } v \notin \mathbb{T}_{u, \bar{d}}\right\} .
$$

The level $k$ rigid stabilizer is the direct product

$$
\operatorname{rist}_{\Gamma}(k)=\prod_{u \in \mathbb{T}_{\bar{d}}^{k}} \operatorname{rist}_{\Gamma}(u)
$$

\section{4 $\quad L^{2}$-isoperimetric profile and return probability}

Given a probability measure $\phi$ on a group $G$, let $\left(S_{n}^{l}\right)_{0}^{\infty}\left(\operatorname{resp},\left(S_{n}^{r}\right)_{0}^{\infty}\right)$ denotes the trajectory of the left (resp. right) random walk driven by $\phi$ (often started at the identity element $e$ ). More precisely, if $\left(X_{n}\right)_{1}^{\infty}$ are independent identically distributed $G$-valued random variables with law $\phi$, then

$$
S_{n}^{l}=X_{n} \ldots X_{1} X_{0} \quad\left(\operatorname{resp} . S_{n}^{r}=X_{0} X_{1} \ldots X_{n}\right) .
$$

Let $\mathbf{P}_{*, \phi}^{x}, *=l$ or $r$ be the associated measure on $G^{\mathbb{N}}$ with $X_{0}=x$ and $\mathbf{E}_{*, \phi}^{x}$ the corresponding expectation $\mathbf{E}_{*, \phi}^{x}(F)=\int_{G^{\mathbb{N}}} F(\omega) d \mathbf{P}_{*, \phi}^{x}(\omega)$. In particular,

$$
\mathbf{P}_{*, \phi}^{e}\left(S_{n}=x\right)=\mathbf{E}_{*, \phi}^{e}\left(\mathbf{1}_{x}\left(S_{n}\right)\right)=\phi^{(n)}(x) .
$$

In this work, we find it convenient to work (mostly, but not always) with the left version of the random walk and we will drop the subscript $l$ in the notation introduced above unless we need to emphasize the differences between left and 
right. Observe that the random walk on the left is a right-invariant process since $\left(X_{n} \ldots X_{0}\right) y=X_{n} \ldots\left(X_{0} y\right)$. When the measure $\phi$ is symmetric in the sense that $\phi(x)=\phi\left(x^{-1}\right)$ for all $x \in G$, its Dirichlet form is defined by

$$
\mathcal{E}_{\phi}(f, f)=\mathcal{E}_{G, \phi}(f, f)=\frac{1}{2} \sum_{x, y \in G}|f(y x)-f(x)|^{2} \phi(y) .
$$

This is the Dirichlet form associated with random walk on the left, $\mathcal{E}_{\phi}^{l}=\mathcal{E}_{\phi}$ and $\mathcal{E}_{\phi}^{r}$ is defined similarly.

Given two functions $f_{1}, f_{2}$ taking real values but defined on an arbitrary domain (not necessarily a subset of $\mathbb{R}$ ), we write $f \asymp g$ to signify that there are constants $c_{1}, c_{2} \in(0, \infty)$ such that $c_{1} f_{1} \leq f_{2} \leq c_{2} f_{1}$. Given two monotone real functions $f_{1}, f_{2}$, write $f_{1} \simeq f_{2}$ if there exists $c_{i} \in(0, \infty)$ such that $c_{1} f_{1}\left(c_{2} t\right) \leq f_{2}(t) \leq c_{3} f_{1}\left(c_{4} t\right)$ on the domain of definition of $f_{1}, f_{2}$ (usually, $f_{1}, f_{2}$ will be defined on a neighborhood of 0 or infinity and tend to 0 or infinity at either 0 or infinity. In some cases, one or both functions are defined only on a countable set such as $\mathbb{N}$ ). We denote the associated order by $\lesssim$. Note that the equivalence relation $\simeq$ distinguishes between power functions of different degrees and between stretched exponentials $\exp \left(-t^{\alpha}\right)$ of different exponent $\alpha>0$ but does not distinguishes between different rates of exponential growth or decay. In this paper, we will consider functions of the type $\exp (-n / \omega(n))$ where $\omega$ is slowly variant at infinity so that $\omega(c t) \sim \omega(t)$ for any fixed $c>0$ and $t$ tending to infinity.

Our main interest in this paper concerns the random walk group invariant $\Phi_{G}$, a positive decreasing function defined on $[0, \infty)$ up to the equivalence relation $\simeq$ and which, according to 25. describes the probability of return of any random walk on the group $G$ driven by a measure $\phi$ that is symmetric, as generating support, and a finite second moment with respect to a fixed word metric on $G$. Namely, for any finitely generated group $G$ and any measure $\phi$ as just described,

$$
\forall n=1,2, \ldots, \quad \phi^{(2 n)}(e)=\mathbf{P}_{\phi}^{e}\left(S_{2 n}=e\right) \simeq \Phi_{G}(n) .
$$

Given a symmetric probability measure $\phi$, set

$$
\Lambda_{2, G, \phi}(v)=\Lambda_{2, \phi}(v)=\inf \left\{\lambda_{\phi}(\Omega): \Omega \subset G,|\Omega| \leq v\right\}
$$

where

$$
\lambda_{\phi}(\Omega)=\inf \left\{\mathcal{E}_{\phi}(f, f): \operatorname{support}(f) \subset \Omega,\|f\|_{2}=1\right\} .
$$

In words, $\lambda_{\phi}(\Omega)$ is the lowest eigenvalue of the operator of convolution by $\delta_{e}-\phi$ with Dirichlet boundary condition in $\Omega$. This operator is associated with the discrete time Markov process corresponding to the $\phi$-random walk killed outside $\Omega$. The function $v \mapsto \Lambda_{2, \phi}(v)$ is called the $L^{2}$-isoperimetric profile or spectral profile of $\phi$ (it really is an iso-volumic profile). The $L^{2}$-isoperimetric profile of a group $G$ is defined as the $\simeq$-equivalence class $\Lambda_{G}$ of the functions $\Lambda_{\phi}$ associated to any symmetric probability measure $\phi$ with finite generating support. 
The $L^{2}$-isoperimetric profile $\Lambda_{2, \phi}$ is related to the analogous $L^{1}$-profile

$$
\begin{aligned}
\Lambda_{1, G, \phi}(v) & =\Lambda_{1, \phi}(v) \\
& =\inf \left\{\frac{1}{2} \sum_{x, y}|f(y x)-f(x)| \phi(y):|\operatorname{support}(f)| \leq v, \quad\|f\|_{1}=1\right\} .
\end{aligned}
$$

Using an appropriate discrete co-area formula, $\Lambda_{1, \phi}$ can equivalently be defined by

$$
\Lambda_{1, \phi}(v)=\inf \left\{|\Omega|^{-1} \sum_{x, y} \mathbf{1}_{\Omega}(x) \mathbf{1}_{G \backslash \Omega}(x y) \phi(y):|\Omega| \leq v\right\} .
$$

If we define the boundary of $\Omega$ to be the set

$$
\partial \Omega=\{(x, y) \in G \times G: x \in \Omega, y \in G \backslash \Omega\}
$$

and set $\phi(\partial \Omega)=\sum_{x \in \Omega, x y \in G \backslash \Omega} \phi(y)$ then $\Lambda_{1, \phi}(v)=\inf \{\phi(\partial \Omega) /|\Omega|:|\Omega| \leq v\}$. It is well-known that

$$
\frac{1}{2} \Lambda_{1, \phi}^{2} \leq \Lambda_{2, \phi} \leq \Lambda_{1, \phi}
$$

Recall that the $\mathrm{F} \varnothing$ lner function $\mathrm{F} \varnothing \mathrm{l}_{G, \phi}$ can be defined by

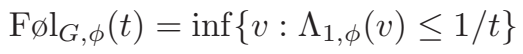

so that $\mathrm{F} \phi \mathrm{l}_{G, \phi}(t)=\Lambda_{1, \phi}^{-1}(1 / t)$ (i.e., $\mathrm{F} \varnothing \mathrm{l}_{G, \phi}$ is the right-continuous inverse of the non-decreasing function $\left.\Lambda_{1, \phi}\right)$.

Notation 2.3. By elementary comparison arguments, for any two symmetric finitely supported probability measure $\phi_{1}, \phi_{2}$ with generating support on a group $G$, we have

$$
\Lambda_{1, G, \phi_{1}} \simeq \Lambda_{1, G, \phi_{2}} \text { and } \Lambda_{2, G, \phi_{1}} \simeq \Lambda_{2, G, \phi_{2}} .
$$

For this reason we often denote by

$$
\Lambda_{1, G}\left(\operatorname{resp} . \quad \Lambda_{2, G}\right)
$$

the $\simeq$-equivalence class of $\Lambda_{1, G, \phi_{1}}\left(\operatorname{resp} . \Lambda_{1, G, \phi_{2}}\right)$ with $\phi$ as above. By abuse of notation, we sometimes write

$$
\Lambda_{p, G}=\Lambda_{p, G, \phi_{1}}
$$

or understand $\Lambda_{p, G}$ as standing for a fixed representative.

Remark 2.4. In the definition of $\Lambda_{p, G, \phi}$ (here, $p=1,2$ ), it is not required that $\phi$ generates $G$. In particular, if $G_{1}$ is a subgroup of a group $G_{2}$ and $\phi$ is a symmetric measure supported on $G_{1}$ then we can consider $\Lambda_{p, G_{i}, \phi}$ for $i=1,2$. Simple considerations imply that $\Lambda_{p, G_{1}, \phi}=\Lambda_{p, G_{2}, \phi}$. In some instance, it might nevertheless be much easier to estimate $\Lambda_{p, G_{2}, \phi}$ than $\Lambda_{p, G_{1}, \phi}$ directly. If $\phi$ is finitely supported and $G_{2}$ is finitely generated then a simple comparison argument yields $\Lambda_{p, G_{1}, \phi} \leq C\left(\phi, G_{1}, G_{2}\right) \Lambda_{p, G_{2}}$. 


\section{A comparison lower bound for $\Lambda$}

Erschler's isoperimetric inequality [10] provides a lower bound on the isoperimetric profile $\Lambda_{1, G, \mu}$ when $G$ is an ordinary wreath product and the measure $\mu$ is well adapted to that structure. See also 27 for corresponding results for $\Lambda_{2, G, \mu}$. It is an important tool which provides an upper bound on the return probability of the random walk driven by $\mu$. However the method does not work directly on permutational wreath products. Here we develop a simple but flexible method based on comparison with product Markov chains. The lower bound on $\Lambda$ obtained in this way is not always sharp, but it still provides useful information in many interesting examples of permutation wreath products, see explicit estimates in Sections 5 and 6 .

We will need the following result regarding the isoperimetric profile of product chains. The statement is an easily consequence of Erschler's isoperimetric inequality on wreath product of Markov chains. Here, for simplicity, we only consider the case needed for our purpose.

Let $X$ be a finite product $X=\prod_{i \in I} H_{i}$, where $I$ is a finite index set and each $H_{i}$ is a copy a given group $H$. On $H$, fix a symmetric probability measure $\eta$. For $i \in I$, let $\eta_{i}$ be the probability measure on $X$ defined by $\eta_{i}(x)=\eta\left(x_{i}\right)$ if $x=\left(x_{j}\right)_{j \in I}$ with $x_{j}=e_{H}$ for $j \neq i$, and $\eta(x)=0$ otherwise.

Let $\zeta$ be the probability measure

$$
\zeta=\frac{1}{|I|} \sum_{i \in I} \eta_{i}
$$

Proposition 3.1. There exists a constant $C_{1}$ such that, for any finite index set $I$, group $H$ and symmetric probability measure $\eta$ as above, and for any positive reals $v_{0}, s$ satisfying $s \in(0,1 / 2]$ and

$$
\Lambda_{1, H, \eta}\left(v_{0}\right)>s
$$

the isoperimetric profile function $\Lambda_{1, X, \zeta}$ satisfies

$$
\Lambda_{1, X, \zeta}(v) \geq s / C_{1} \text { for any } v \leq C_{1}^{-1}\left(v_{0}\right)^{|I| / C_{1}} .
$$

Proof. Equip the index set $I$ with, say, a cyclic group structure and its complete graph structure (including self-loops). Embed the product $\prod_{i \in I} H_{i}$ into the wreath product $\left\{H_{i}\right\} \imath I$ with $H_{i}$ as lamps over $i$ and $I$ as base. See [11. Now, $\prod_{i \in I} H_{i}$ can be thought of as the space of lamp configurations. Let $Y \subset \prod_{i \in I} H_{i}$ be a finite subset. Define

$$
\widetilde{Y}=\{(f, i): f \in Y, i \in I\} .
$$

Consider the transition kernel $\bar{p}$ on $\left\{H_{i}\right\}$ 乙 I,

$$
\bar{p}\left((f, i),\left(f^{\prime}, i^{\prime}\right)\right)=\left\{\begin{array}{cl}
\frac{1}{2} \eta_{i}\left(f^{\prime}(i) f(i)^{-1}\right) & \text { if } i=i^{\prime} \text { and } f=f^{\prime} \text { except at } i, \\
\frac{1}{2|I|} & \text { if } f=f^{\prime} .
\end{array}\right.
$$


Then

$$
\bar{p}\left(\tilde{Y}, \tilde{Y}^{c}\right)=\frac{1}{2}|I| \zeta(\partial Y) .
$$

In the base $I$ equipped with its complete graph structure, any set $U$ with $u$ elements has boundary of weight $|I|^{-1} u(|I|-u)$ so that $\Lambda_{1, I}(u) \simeq(1-u /|I|)$.

Thus [11, Theorem 1] together with the hypotheses that $\Lambda_{1, H, \eta}\left(v_{0}\right)>s$ and the fact that $\Lambda_{1, I}^{-1}(s) \geq|I|(1-s)$ yield that

$$
\frac{\bar{p}\left(\tilde{Y}, \tilde{Y}^{c}\right)}{|\tilde{Y}|}<s / K \text { implies }|\tilde{Y}| \geq\left(v_{0}\right)^{(1-s)|I| / K} .
$$

Since

$$
|I||Y|=|\tilde{Y}| \text { and } \frac{\zeta(\partial Y)}{|Y|}=\frac{\bar{p}\left(\tilde{Y}, \tilde{Y}^{c}\right)}{2|\tilde{Y}|}
$$

we obtain

$$
\Lambda_{1, X, \zeta}(v) \geq s / K \text { for any } v \leq \frac{1}{|I|}\left(v_{0}\right)^{|I| /(2 K)}
$$

because $s \in(0,1 / 2]$. The desired inequality follows by choosing $C_{1}>K$ large enough.

Remark 3.2. Proposition 3.1 concerns expansion of small sets in $X$. When $X$ is a finite set, the proposition does not provide information about expansion of large sets or the spectral gap. For example suppose that for each $i \in I, H_{i}=\mathbb{Z}_{2}$, the bound on $v$ in Proposition 3.1 is $C_{1}^{-1} 2^{|I| / C_{1}}=C_{1}^{-1}|X|^{1 / C_{1}}$. Therefore the stated isoperimetric inequality is only effective for small sets.

When $H=\mathbb{Z}_{2}$ or $\mathbb{Z}$, more precise estimates are known thanks to the sharp isoperimetric inequalities on hypercubes and Euclidean lattices.

Example 3.3. For the hypercube $X=\mathbb{Z}_{2}^{|I|}, \eta(1)=1$, from edge expansion results on the hypercube, we have (see, e.g., [17]),

$$
\Lambda_{1, X, \zeta}(v) \geq 1-\frac{\log _{2} v}{|I|} .
$$

Thus, for any $K>1$ and $v \leq 2^{|I| / K}$,

$$
\Lambda_{1, X, \zeta}(v) \geq 1-\frac{1}{K} .
$$

This is to be compared with the conclusion provided by Proposition 3.1 which states that

$$
\Lambda_{1, X, \zeta}(v) \geq C_{1}^{-1} \text { for all } v \leq C_{1}^{-1} 2^{|I| / C_{1}} .
$$

Example 3.4. For the Euclidean lattice $X=\mathbb{Z}^{|I|}, \eta( \pm 1)=1 / 2$, the sharp discrete isoperimetric inequality states that for any finite set $A$ (see, e.g., [22, Theorem 6.22])

$$
2\left|\partial_{\zeta} A\right| \geq|A|^{\frac{|I|-1}{|I|}} .
$$


Therefore

$$
\frac{\left|\partial_{\zeta} A\right|}{|A|} \leq s \text { implies }|A| \geq(1 /(2 s))^{|I|} .
$$

Equivalently,

$$
\Lambda_{1, \mathbb{Z}|I|, \zeta}(v) \geq s \text { for all } v \leq(1 /(2 s))^{|I|} .
$$

In this case, Proposition 3.1 gives the weaker statement that

$$
\Lambda_{1, \mathbb{Z}|I|, \zeta}(v) \geq s / C_{1} \text { for all } v \leq C_{1}^{-1}(1 / s)^{|I| / C_{1}} .
$$

For applications we have in mind, it is useful to consider the case when $\eta$ is a spread-out measure as in the following example.

Example 3.5. On the Euclidean lattice $X=\mathbb{Z}^{|I|}$, let $\eta=\frac{1_{[-|I|,|I|]}}{2|I|+1}$, i.e., $\eta$ is uniform on the interval $[-|I|,|I|]$ in $\mathbb{Z}$. In this case, Proposition 3.1 yields

$$
\Lambda_{1, X, \zeta}(v) \geq 1 / C_{1} \text { for all } v \leq C_{1}^{-1}|I|^{|I| / C_{1}} .
$$

The important point here is that this estimate is uniform in $|I|$.

Proposition 3.6. There exists a constant $C_{1}$ such that the following holds. Let $G$ be a finitely generated group equipped with a finite symmetric generating set $S$. Suppose $X$ is a subgroup of $G$ of the form $X=\prod_{i \in I} H_{i}$ with $H_{i} \simeq H$ for some group $H$. Let $\eta$ be a symmetric probability measure on $H$ with the property that

$$
\Lambda_{1, H, \eta}\left(v_{0}\right) \geq s_{0}
$$

for positive real $v_{0}, s_{0}$ with $s_{0} \in(0,1 / 2]$. Let $\zeta$ be defined in terms of $\eta$ by (3.1) and assume further that

$$
\max _{g \in \operatorname{Supp}(\zeta)}|g|_{(G, S)} \leq R
$$

Then, letting $\mathbf{u}_{1}$ be the uniform probability measure on $\{e\} \cup S$, we have

$$
\Lambda_{1, G, \mathbf{u}_{1}}(v) \geq \frac{1}{C_{1}|S| R}, \text { for } v \leq C_{1}^{-1}\left(v_{0}\right)^{|I| / C_{1}} .
$$

Proof. This follows immediately from Proposition 3.1 together with an easy comparison argument to pass from the measure $\zeta$ defined at (3.1) to the uniform probability measure $\mathbf{u}_{1}$ on $\{e\} \cup S$.

Example 3.7. We show how this bound works on ordinary wreath products. Consider the wreath product $G=H \imath \Gamma$, where $H$ and $\Gamma$ are finitely generated groups. Given a radius $R$, a natural choice for $X$ is provided by the lamps over the ball of radius $R$ in the base group $\Gamma$, that is

$$
X=\prod_{x \in B_{\Gamma}\left(e_{\Gamma}, R\right)}(H)_{x} .
$$


Take $\eta=\mathbf{u}_{H, R}$ to be the uniform measure on the ball of radius $R$ in $H$ centered at $e_{H}$. Then Proposition 3.6 implies that

$$
\Lambda_{1, G, \mathbf{u}_{1}}(v) \geq \frac{1}{C_{1} R}, \text { for all } v \leq C_{1}^{-1}\left(\left|B_{H}\left(e_{H}, R\right)\right|\right)^{\left|B_{\Gamma}\left(e_{\Gamma}, R\right)\right| / C_{1}} .
$$

In the case when both $\Gamma$ and $H$ are groups whose isoperimetric function is sharply determined by the volume growth then this bound is sharp and is equivalent to the bound provided directly by Erschler's inequality. For instance, if $\Gamma=H=\mathbb{Z}$ so that $G=\mathbb{Z} \imath \mathbb{Z}$, this gives

$$
\Lambda_{1, \mathbb{Z} Z \mathbb{Z}}(v) \geq c_{1} \frac{\log \log v}{\log v} .
$$

Now, we show that a similar bound works on the permutational wreath products $G=H \imath_{\mathcal{S}} \Gamma$ where $\Gamma, H$ are finitely generated groups equipped with finite symmetric generating sets $S_{\Gamma}, S_{H}$.

Let $x \in \mathcal{S}$ be a vertex in the Schreier graph, let $g \in \Gamma$ be a group element such that

$$
|g|_{(\Gamma}=d_{\mathcal{S}}(o, x), g \cdot o=x .
$$

Then

$$
\left(\mathbf{1}_{h}^{x}, e_{\Gamma}\right)=\left(\mathbf{e}_{H}^{\mathcal{S}}, g\right)\left(\mathbf{1}_{h}^{o}, e_{\Gamma}\right)\left(\mathbf{e}_{H}^{\mathcal{S}}, g^{-1}\right)
$$

thus

$$
\left|\left(\mathbf{1}_{h}^{x}, e_{\Gamma}\right)\right|_{G} \leq 2|g|_{\Gamma}+|h|_{H} .
$$

Let $\mathbf{u}_{\Gamma, r}$ (resp. $\mathbf{u}_{H, r}$ ) denote the uniform probability measure on the ball $B_{\Gamma}\left(e_{\Gamma}, r\right)$ in $\Gamma\left(\operatorname{resp} . B_{H}\left(e_{H}, r\right)\right.$ in $\left.H\right)$.

Corollary 3.8. Referring to the setting introduced above, Let $\mathfrak{q}=\frac{1}{2}\left(\mathbf{u}_{\Gamma, 1}+\mathbf{u}_{H, 1}\right)$ on $G=H$ is $_{\mathcal{S}} \Gamma$. For each $r$, let $\eta_{r}$ be a symmetric probability measure on $H$ with support in the ball $B\left(e_{H}, r\right)$. Then

$$
\Lambda_{1, G, \mathfrak{q}}(v) \geq \frac{1}{C r} \text { for } v \leq C^{-1}\left(\Lambda_{1, H, \eta_{r}}^{-1}(1 / 2)\right)^{\left|B_{\mathcal{S}}(o, r)\right| / C} .
$$

Proof. Consider the measure

$$
\zeta=\frac{1}{\left|B_{\mathcal{S}}(o, r)\right|} \sum_{x \in B_{\mathcal{S}}(o, r)}\left(\eta_{r}\right)_{x} .
$$

then by (3.3) we have

$$
\max _{g \in \operatorname{supp}(\zeta)}|g|_{G} \leq 3 r .
$$

The conclusion follows from Proposition 3.6

Example 3.9. On the permutational wreath product $G=(\mathbb{Z} / 2 \mathbb{Z}) \imath_{\mathcal{S}} \Gamma$, we have a lower bound for $L^{1}$-isoperimetric profile

$$
\Lambda_{1, G}(v) \geq \frac{1}{C_{1} r} \text { for } v \leq C_{1}^{-1} 2^{\left|B_{\mathcal{S}}(o, r)\right| / C_{1}} .
$$


If instead take $\tilde{G}=\mathbb{Z} \imath_{\mathcal{S}} \Gamma$, we take $\eta_{r}$ to be the uniform probability on $\mathbb{Z} \cap[-r, r]$. This yields

$$
\Lambda_{1, \widetilde{G}}(v) \geq \frac{1}{C_{1} r} \text { for } v \leq C_{1}^{-1} r^{\left|B_{\mathcal{S}}(o, r)\right| / C_{1}} .
$$

For instance, when $\Gamma$ is the infinite Dihedral group $D_{\infty}$ of Example 2.2 this gives

$$
\Lambda_{1,(\mathbb{Z} / 2 \mathbb{Z}) \imath D_{\infty}}(v) \gtrsim \frac{1}{\log v} \text { and } \Lambda_{1, \mathbb{Z} \imath D_{\infty}}(v) \gtrsim \frac{\log \log v}{\log v} .
$$

More interesting examples are provided by [5]. Bondarenko proves that any group $\Gamma$ generated by a polynomial automaton of degree $d$ has a defining marked Schreier graph $(\mathcal{S}, o)$ such that $V_{\mathcal{S}}(o, r) \leq A^{(\log r)^{d+1}}$. The estimate is sharp ([5. Theorem 4]) in the sense that Bondarenko gives examples of degree $d$ automaton groups for which $B^{(\log r)^{d+1}} \leq V_{\mathcal{S}}(o, r) \leq A^{(\log r)^{d+1}}$ for some $1<$ $B \leq A<\infty$. The so-called long range group belong to this class, with degree $d=1$. See also 1. For such a group $\Gamma$, the Schreier graph volume lower bound $B^{(\log r)^{d+1}} \leq V_{\mathcal{S}}(o, r)$ yields (with lamp group $H$ equals to $\mathbb{Z} / 2 \mathbb{Z}$ or $\mathbb{Z}$ or any group of polynomial volume growth),

$$
\Lambda_{1, H \iota_{\mathcal{S}} \Gamma}(v) \geq \exp \left(-C(\log \log v)^{1 /(d+1)}\right)
$$

and, consequently,

$$
\Phi_{H \backslash \mathcal{S} \Gamma}(n) \leq \exp \left(-\frac{n}{\exp \left(C(\log n)^{1 /(d+1)}\right)}\right) .
$$

Regarding $\Lambda_{2}$ upper bounds and $\Phi$ lower bounds, we note that [1] shows that any degree $d$ automaton group embeds into an appropriate degree $d$ mother group. For degree $d=0,1,2$, the mother groups can be studied by the technique of section 4 as long as some appropriate "resistance estimates" can be derived for the associated Schreier graph. We will not pursue this here, in part because none of the resulting bounds appear to capture the real behavior of $\Lambda_{1}, \Lambda_{2}$ and $\Phi$ for these examples. We note the degree $d$ automaton groups are known to be amenable for $d=0,1,2$ and that the amenability question is open in degree greater than 2 .

Proposition 3.10. On the permutational wreath product $G=H \imath_{\mathcal{S}} \Gamma$ with $\Gamma, H$ finitely generated, let $\mathfrak{q}=\frac{1}{2}(\mu+\nu)$, where $\mu=\mathbf{u}_{\Gamma, 1}$ on $\Gamma$ and $\nu$ is a symmetric probability measure on $H$. Then the $L^{2}$-isoperimetric profile of $\mathfrak{q}$ on $G$ satisfies

$$
\Lambda_{2, G, q}(v) \geq \frac{1}{C_{1} r^{2}} \text { for } v \leq C^{-1}\left(\Lambda_{2, H, \nu}^{-1}\left(1 / r^{2}\right)\right)^{\left|B_{\mathcal{S}}(o, r)\right| / C} .
$$

Proof. For the symmetric probability measure $\nu$ on $H$ with $L^{2}$-isoperimetric profile $\Lambda_{2, H, \nu}$ and any $r>1$, [27, Theorem 4.7] provides a symmetric probability measure $\eta_{r}$ (it depends very much on $\nu$ as well) such that

$$
\mathcal{E}_{H, \nu} \geq c r^{-2} \mathcal{E}_{H, \eta_{r}}
$$


and

$$
\Lambda_{2, H, \eta_{r}}(v) \geq 1 / 2 \text { for all } v \leq C^{-1}\left(\Lambda_{2, H, \nu}\left(1 / r^{2}\right)\right)^{\left|B_{\mathcal{S}}(o, r)\right| / C} .
$$

Consider the symmetric probability measure

$$
\zeta_{r}=\frac{1}{\left|B_{\mathcal{S}}(o, r)\right|} \sum_{x \in B_{\mathcal{S}}(o, r)}\left(\eta_{r}\right)_{x}
$$

Proposition 3.1 applied to $\zeta_{r}$ provides the estimate

$$
\Lambda_{2, G, \zeta_{r}}(v) \geq 1 / C \text { for all } v \leq C^{-1}\left(\Lambda_{2, H, \nu}\left(1 / r^{2}\right)\right)^{\left|B_{\mathcal{S}}(o, r)\right| / C}
$$

for some large constant $C>$. Further, (3.2) and the property $\mathcal{E}_{H, \nu} \geq c r^{-2} \mathcal{E}_{H, \eta_{r}}$ show that (with a different constant $c>0$ )

$$
\mathcal{E}_{G, \mathfrak{q}} \geq c^{\prime} r^{-2} \mathcal{E}_{G, \zeta_{r}} .
$$

Putting these two estimates together provides the desired conclusion.

The next proposition applies the technique of this section in the context of finitely generated subgroups of the automorphism group of a rooted tree $\mathbb{T}_{\bar{d}}$.

Proposition 3.11. Let $\Gamma$ be a finitely generated subgroup of Aut $\left(\mathbb{T}_{\bar{d}}\right)$ as in Section 2.3. Let $u=x_{0} x_{1} \ldots x_{n-1}$ be a vertex in level $n$ of the tree $\mathbb{T}_{\bar{d}}$, and let $\mathcal{S}_{n}(u)$ be the orbital Schreier graph of $u$ under action of $\Gamma$. Let $\rho_{n} \in \operatorname{rist}_{\Gamma}(u)$ be a nontrivial element, $\rho_{n} \neq e_{\Gamma}$, of length $\left|\rho_{n}\right|_{\Gamma}$ in $\Gamma$. Then there exists a constant $C \geq 1$ such that

$$
\Lambda_{1, \Gamma}(v) \geq \frac{1}{C \max \left\{\left|\rho_{n}\right|_{\Gamma}, r\right\}} \text { for all } v \leq C^{-1} 2^{\left|B_{\mathcal{S}_{n}(u)}(u, r)\right| / C} .
$$

Proof. For every vertex $v \in \mathcal{S}_{n}(u)$, fix an element $g^{v} \in \Gamma$ such that $g^{v} \cdot u=v$ and

$$
\left|g^{v}\right|_{\Gamma}=d_{\mathcal{S}_{n}(u)}(u, v) .
$$

In terms of sections and permutations at level $n$ (see Section 2.3), $g^{v}$ is

$$
g^{v}=\left(g_{x}^{v}\right)_{x \in \mathbb{T}^{n}} \sigma^{v}
$$

where as

$$
\rho_{n}=\left(\tilde{\rho}_{x}\right)_{x \in \mathbb{T}^{n}} \text { id }
$$

where $\tilde{\rho}_{x}$ is the identity except for $x=u$ where $\tilde{\rho}_{x}=\rho_{n}(u)$. Then

$$
g^{v} \rho_{n}\left(g^{v}\right)^{-1}=\left(e, . ., e, g_{\sigma^{v}(u)}^{v} \rho_{n}(u) g_{\sigma^{v}(u)}^{-1}, e, \ldots, e\right) \text { id }
$$

where the only nontrivial section is at site $\sigma^{v}(u)=v$. Since $\rho_{n}$ is nontrivial, it follows that the conjugation $g_{v}^{v} \rho_{n}\left(g_{v}^{v}\right)^{-1}$ is also a nontrivial element in $\operatorname{rist}_{\Gamma}(v)$.

Now, let $\zeta$ be the symmetric probability measure on the subgroup

$$
\left\langle g^{v} \rho_{n}\left(g^{v}\right)^{-1}: v \in B(u, r)\right\rangle
$$


defined be

$$
\zeta(\gamma)=\frac{1}{2\left|B_{\mathcal{S}_{n}(u)}(u, r)\right|} \sum_{v \in B_{\mathcal{S}_{n}(u)}(u, r)} \mathbf{1}_{\left\{g^{v} \rho_{n}^{ \pm 1}\left(g^{v}\right)^{-1}\right\}}
$$

Then $\zeta$ has the form (3.1) on the product

$$
X=\prod_{v \in B_{\mathcal{S}_{n}(u)}(u, r)} \operatorname{rist}_{\Gamma}(v) .
$$

The desired result follows by comparison between simple random walk on $\Gamma$ and $\zeta$ together with Proposition 3.1 .

In [12, Erschler uses vertex stabilizers to estimate the Følner function of certain groups. It is harder to reach a rigid stabilizer but, if one does, one can make use of the product structure as described in Proposition 3.11. For a concrete application of Proposition 3.11, see Corollary 6.4.

\section{Upper-bound for $\Lambda$}

In this section we present a technique that provides an upper-bound on the $L^{2}$ isoperimetric profile $\Lambda_{2, G, \mathfrak{q}}$ when $G=H \imath_{\mathcal{S}} \Gamma$ is a permutation wreath product with infinite amenable lamp group $H$ (e.g., $H=\mathbb{Z}$ ) and the action of $\Gamma$ on $\mathcal{S}$ is extensively amenable in the sense of [18].

Definition 4.1 ([18, Definition 1.1]). The action of a group $\Gamma$ on a set $\mathcal{S}$ is extensively amenable if there is a $\Gamma$-invariant mean on $\mathcal{P}_{f}(\mathcal{S})$ giving full weight to the collection of subsets that contain an arbitrary given element of $\mathcal{P}_{f}(\mathcal{S})$.

This property appears implicitly in [19] where it is used to prove the amenability of the topological full group of any minimal Cantor system. This argument was later extended in [20, 18].

Here we use a basic version of this idea to obtain explicit upper-bound on the $L^{2}$-isoperimetric profile $\Lambda_{2, G, \mathfrak{q}}$. Qualitatively, this is equivalent to giving upper bound on the Følner function.

\subsection{A unifying framework}

Let $\Gamma$ be a finitely generated group acting on a space $X$. In the examples we consider, $X$ will be a countable set, the vertex set of a graph. Let

$$
S=\left\{s_{1}^{ \pm 1}, \ldots, s_{k}^{ \pm 1}\right\}
$$

be a finite symmetric generating set of $\Gamma$. In what follows we need to also consider the finite alphabet of $2 k$ distinct letters

$$
\mathbf{S}=\left\{s_{1}^{ \pm 1}, \ldots, s_{k}^{ \pm 1}\right\}
$$


Note that we do use the same notation $s_{i}^{ \pm 1}$ for the letters and their evaluation in $\Gamma$. We let

$$
\mathbf{S}^{(\infty)}
$$

be the set of all finite words on $\mathbf{S}$.

Let $o$ be a point in $X$ chosen as a reference point. Let $\mathcal{S}$ denote the orbital Schreier graph of $o$ under the action of $\Gamma$. It is well understood that the inverted orbits of certain points in $X$ play a key role in understanding the group $\Gamma$, see for example 2. In some examples we will need to keep track of inverted orbits of more than one point.

Notation 4.2 (Inverted orbit, $\mathcal{O}(w ; J), \Omega(J, A, B)$ ). Let $w=w_{1} w_{2} \ldots w_{l}, w_{i} \in \mathbf{S}$, be an arbitrary word of length $l$.

- Given $x \in X$, the inverted orbit $\mathcal{O}(w ; x)$ of $x$ under $w$ is the subset of $X$ defined by

$$
\mathcal{O}(w ; x)=\left\{w_{1} \cdots w_{l} \cdot x, w_{1} \cdots w_{l-1} \cdot x, \ldots, w_{1} \cdot x, x\right\} .
$$

- More generally, given a countable subset $J$ of $X$, set

$$
\mathcal{O}(w ; J)=\bigcup_{x \in J} \mathcal{O}(w, x) .
$$

- For any two subsets $J \subset B \subset X$, define $\Omega(J, B)$ to be the subset of $\mathbf{S}^{(\infty)}$ defined by

$$
\Omega(J, B):=\left\{w \in \mathbf{S}^{(\infty)}: \mathcal{O}(w, J) \subset B\right\} .
$$

Let $\bar{\Omega}(J, B) \subset \Gamma$ be the image of $\Omega(J, B) \subset \mathbf{S}^{(\infty)}$ under evaluation in $\Gamma$.

Definition $4.3\left((J, B)\right.$-admissible function). Equip $\mathcal{P}_{f}(X)$ with the usual counting measure. Given subsets $J \subset B$ of $X$, we say that a function

$$
F: \mathcal{P}_{f}(X) \rightarrow \mathbb{R}
$$

is $(J, B)$-admissible if there exist a subset $A \subset X$ such that for any $Y \in \operatorname{supp}(F)$ we have $Y=g \cdot A$ for some $g \in G$ and $J \subset Y=g \cdot A \subset B$.

Definition 4.4 (Hypothesis $(\Omega)$ ). We say that $(\Gamma, X, o)$ satisfies $(\Omega)$ if the following conditions hold:

1. There exists a sequence of couples $\left(\left(J_{n}, B_{n}\right)\right)$ where $J_{n}, B_{n}$ are finite subsets of $\mathcal{S}$ such that

$$
o \in J_{n} \subset B_{n} \text { and } \bigcup_{n} \bar{\Omega}\left(J_{n}, B_{n}\right)=\Gamma .
$$

2. There exists a sequence $\left(\boldsymbol{\Gamma}^{n}\right)$ of amenable groups and maps

$$
\vartheta_{n}: \Omega\left(J_{n}, B_{n}\right) \rightarrow \Gamma^{n}
$$

such that 
- For any $s \in \mathbf{S}$ and integer $n$, we have $s, s^{-1} \in \Omega\left(J_{n}, B_{n}\right)$.

- For any pair $w_{1}, w_{2} \in \Omega\left(J_{n}, B_{n}\right)$ whose evaluations in $\Gamma$ are equal, we have

$$
\vartheta_{n}\left(w_{1}\right)=\vartheta_{n}\left(w_{2}\right) .
$$

- For any pair $w_{1}, w_{2}$ such that $w_{1}, w_{2}, w_{1} w_{2} \in \Omega\left(J_{n}, B_{n}\right)$, we have

$$
\vartheta_{n}\left(w_{1} w_{2}\right)=\vartheta_{n}\left(w_{1}\right) \vartheta_{n}\left(w_{2}\right) .
$$

Remark 4.5. Under Hypothesis $(\Omega)$, each of the map $\vartheta_{n}$ induces an injective map from $\bar{\Omega}\left(J_{n}, B_{n}\right)$ to $\Gamma^{n}$. For this reason we will refer to $\vartheta_{n}$ as the "local embeddings" provided by Hypothesis $(\Omega)$.

The point of the hypothesis $(\Omega)$ is that the groups $\Gamma^{n}$ are potentially much easier to understand than the group $\Gamma$. Typically, the map $\vartheta_{n}$ cannot be extended globally to $\Gamma$, but in computations when we can restrict attention to the finite subset $\bar{\Omega}\left(J_{n}, B_{n}\right)$ of $\Gamma$, elements there can be identified with elements in $\Gamma^{n}$ via the map $\vartheta_{n}$. This will allow us to combine functions on $\mathcal{P}_{f}(\mathcal{S})$ and functions on $\Gamma^{n}$ to bound the isoperimetric profile $\Lambda_{\mathbb{Z} \imath_{\mathcal{S}} \Gamma}$ of the permutation wreath product $\mathbb{Z} \imath_{\mathcal{S}} \Gamma$.

Example 4.6. Consider the Dihedral group $D_{\infty}=<s, t>$ of Example 2.2 defined in terms of the Schreier graph of Figure 6. We show how hypothesis $(\Omega)$ is satisfies in this simple case. Set $J=\{o=0\}$ be the left-most vertex in $\mathcal{S}=\{0,1,2, \ldots\}$ and set $B_{n}=\left\{0, \ldots, 2^{n}-1\right\}$. The set $\Omega\left(o, B_{n}\right)$ is the collection of words $w=w_{1} \ldots w_{i}$ on $s^{ \pm 1}$ and $t^{ \pm 1}$ so that for any $j \leq i$, the associated reduced word on $s, t$ (each of order two) is of length at most $2^{n}$ if the reduced word ends in $t$ and $2^{n}-1$ if the reduced word end in $s$.

For $\boldsymbol{\Gamma}^{n}$, take the finite dihedral group $D_{2^{n}}$ as discussed in Example 2.1 and let $\vartheta_{n}$ be the natural projection map $\mathbf{S}^{(\infty)} \rightarrow D_{\infty} \rightarrow D_{2^{n}}$. The required properties are easily seen to be satisfied.

Recall that the elements of the group $G=\mathbb{Z} \imath_{\mathcal{S}} \Gamma$ are pairs $(\Upsilon, g)$ where $g \in \Gamma$ and $\Upsilon$ is a finitely supported $\mathbb{Z}$-lamp configuration on $\mathcal{S}$. The action of $\Gamma$ on $X$ is easily extended to an action of $G$ by setting $(\Upsilon, g) \cdot x=g \cdot x$ for all $(\Upsilon, g) \in G$ and $x \in X$.

Lemma 4.7 (Lamps control inverted orbits). Assume that $(\Gamma, X, o)$ satisfies Hypothesis $(\Omega)$. Fix a function $F_{n}$ on $\mathcal{P}_{f}(\mathcal{S})$ which is $\left(J_{n}, B_{n}\right)$-admissible. Set

$$
\widetilde{U}_{n}=\left\{f \in \oplus_{x \in \mathcal{S}}(\mathbb{Z})_{x}: \operatorname{supp}(f) \in \operatorname{supp}\left(F_{n}\right)\right\} .
$$

For each integer $n$, consider $g_{n, 0}=\left(\Upsilon_{n, 0}, e\right) \in \mathbb{Z} \imath_{\mathcal{S}} \Gamma$ and a sequence $\left(g_{i}\right)_{i \geq 1}$ of group elements in $\mathbb{Z} \imath_{\mathcal{S}} \Gamma$ with $g_{i} \in\left\{\left( \pm \mathbf{1}_{1}^{o}, e\right)\right\} \cup S$ for all $i \geq 1$. Set

$$
g_{k} \ldots g_{1} g_{n, 0}=\left(\Upsilon_{n, k}, \gamma_{n, k}\right) \text {. }
$$

Assume that for some $m$,

$$
\forall k=0,1, \ldots, m, \Upsilon_{n, k} \in \widetilde{U}_{n} .
$$


Then, considering the $g_{j}$ both as formal letters and group elements,

$$
\mathcal{O}\left(g_{m} \ldots g_{1} ; J_{n}\right) \subset \operatorname{supp}\left(\Upsilon_{n, m}\right)=g_{m} \ldots g_{1} \cdot \operatorname{supp}\left(\Upsilon_{0}\right) .
$$

Proof. In this lemma, $n$ is fixed so, for the proof, we drop the reference to $n$. The proof is by induction on $m$. The claim is obviously true for $m=0$ given the choice of $\Upsilon_{0}$. Assume that the property holds for length $m$. For $m+1$, we discuss two cases separately.

In the first case, when $g_{m+1} \in\left\{ \pm \mathbf{1}_{1}^{o}\right\}$, the move $g_{m+1}$ does not change the inverted orbit of the trajectory, that is,

$$
\mathcal{O}\left(g_{m+1} \ldots g_{1} ; J\right)=\mathcal{O}\left(g_{m} \ldots g_{1} ; J\right) \text {. }
$$

By the induction hypothesis

$$
\mathcal{O}\left(g_{m} \ldots g_{1} ; J\right) \subset \operatorname{supp} \Upsilon_{m}=g_{m} \ldots g_{1} \cdot \operatorname{supp}\left(\Upsilon_{0}\right) .
$$

From the definition of $\widetilde{U}$ and since, by assumption, $\Upsilon_{m+1} \in \widetilde{U}$, we must have $\operatorname{supp}\left(\Upsilon_{m+1}\right)=g \cdot \operatorname{supp}\left(\Upsilon_{0}\right)$ for some $g \in \Gamma$. This implies that $\left|\operatorname{supp}\left(\Upsilon_{m+1}\right)\right|=$ $\left|\operatorname{supp}\left(\Upsilon_{m}\right)\right|$ and it follows that $g_{m+1}$ did not produce any change in the support of the lamp configuration, that is

$$
\operatorname{supp}\left(\Upsilon_{m+1}\right)=\operatorname{supp}\left(\Upsilon_{m}\right)=g_{m} \ldots g_{1} \cdot \operatorname{supp}\left(\Upsilon_{0}\right)=g_{m+1} \ldots g_{1} \cdot \operatorname{supp}\left(\Upsilon_{0}\right) .
$$

It follows that

$$
\mathcal{O}\left(g_{m+1} \ldots g_{1} ; J\right) \subset \operatorname{supp} \Upsilon_{m+1}=g_{m+1} \ldots g_{1} \cdot \operatorname{supp}\left(\Upsilon_{0}\right)
$$

as desired.

In the second case, when $g_{m+1} \in S$, the induction hypothesis gives

$$
\mathcal{O}\left(g_{m} \ldots g_{1} ; J\right) \subset \operatorname{supp}\left(\Upsilon_{m}\right)=g_{m} \ldots g_{1} \cdot \operatorname{supp}\left(\Upsilon_{0}\right) .
$$

Also, we have

$$
\mathcal{O}\left(g_{m+1} \ldots g_{1} ; J_{n}\right)=J \cup g_{m+1} \cdot \mathcal{O}\left(g_{m} \ldots g_{1} ; J\right)
$$

and

$$
\operatorname{supp}\left(\Upsilon_{m+1}\right)=g_{m+1} \cdot \operatorname{supp}\left(\Upsilon_{m}\right) .
$$

Since $F$ is $(J, B)$-admissible and by assumption, $\Upsilon_{m+1}=g_{m+1} \cdot \Upsilon_{m} \in \widetilde{U}$, it follows that $g_{m+1} \cdot \operatorname{supp}\left(\Upsilon_{m}\right) \in \operatorname{supp}(F)$ and $J \subset g_{m+1} \cdot \operatorname{supp}\left(\Upsilon_{m}\right)$. Therefore, we have

$$
\begin{aligned}
\mathcal{O}\left(g_{m+1} \ldots g_{1} ; J_{n}\right) & =J \cup g_{m+1} \cdot \mathcal{O}\left(g_{m} \ldots g_{1} ; J\right) \\
& \subset J \cup\left(g_{m+1} \cdot \operatorname{supp}\left(\Upsilon_{m}\right)\right) \\
& =\operatorname{supp}\left(\Upsilon_{m+1}\right)=g_{m+1} \ldots g_{1} \cdot \operatorname{supp}\left(\Upsilon_{0}\right) .
\end{aligned}
$$

Combining the two cases, the lemma follows. 


\subsection{Comparison of two local graph structures}

In this section we compare two graph structures, one on $G=\mathbb{Z}$ is $_{\mathcal{S}} \Gamma$ and the other on product set $\left(\oplus_{x \in \mathcal{S}}(\mathbb{Z})_{x}\right) \times \Gamma^{n}$. Our goal is to show that certain certain subgraphs in these two graphs are isomorphic.

In $G=\mathbb{Z} \imath_{\mathcal{S}} \Gamma$, let $Q=\left\{\left( \pm \mathbf{1}_{1}^{o}, e_{\Gamma}\right)\right\} \cup S$ be the standard switch-or-walk generating set (recall that $S$ is a finite symmetric generating set of $\Gamma$ ). The (left) directed Cayley graph of $G$ with respect to $Q$ is obtained by putting a directed edge $(g, q g)$ between $g \in G$ and $q g$ where $q \in Q$. The edge $(g, q g)$ is labeled by $q$, where $q \in Q$ is either a move induced by $\mathbf{1}_{1}^{o}$ in the lamp configuration, or a move induced by $s \in S$. We write $(G, Q)$ for this directed labeled Cayley graph of $G$ with respect to generating set $Q$.

On the product $\left(\oplus_{x \in \mathcal{S}}(\mathbb{Z})_{x}\right) \times \Gamma^{n}$, define a graph structure by connecting vertices with one of the following two types of edges:

- connect $(f, \gamma)$ and $\left(f \pm \mathbf{1}_{1}^{o}, \gamma\right)$ with a directed edge labeled with $\pm \mathbf{1}_{1}^{o}$;

- connect $(f, \gamma)$ and $\left(s^{-1} \cdot f, \vartheta_{n}(s) \gamma\right)$ with a directed edge labeled with $s \in$ $S$.

We denote the resulting directed graph by $\left(\left(\oplus_{x \in \mathcal{S}}(\mathbb{Z})_{x}\right) \times \Gamma^{n}, P\right)$. Note that it is not necessarily connected.

On both graphs edges are labeled by either with $\mathbf{1}_{1}^{o}$ (which corresponds to changing the lamp value at $o$ ) or with a generator $s \in S$. Given a word $\omega=p_{l} \ldots p_{1}$ in the alphabet $Q$, it makes sense to follow $\omega$ as paths on both graphs. Starting from the same initial lamp configuration on both graphs, from definition of the graph structures, following the same word $\omega$, the trajectories of the lamp function $\Upsilon_{k}$ are exactly the same on the two graphs.

Notation 4.8 (Definition of $\mathcal{G}_{n}\left(\Upsilon_{0}\right)$ and $\Gamma^{n}\left(\Upsilon_{0}\right)$ ). Let $F_{n}$ be an $\left(J_{n}, B_{n}\right)$ admissible function. Let $\Upsilon_{0} \in \oplus_{x \in \mathcal{S}}(\mathbb{Z})_{x}$ be $\operatorname{such}$ that $\operatorname{supp}\left(\Upsilon_{0}\right) \in \operatorname{supp}\left(F_{n}\right)$.

- On the graph $(G, Q)$ consider the subgraph with vertex set

$$
\left\{(f, \gamma): \operatorname{supp}(f) \in \operatorname{supp}\left(F_{n}\right)\right\}
$$

and edge set made of those original edges of $(G, Q)$ that connects points in the selected vertex set. Let $\mathcal{G}_{n}\left(\Upsilon_{0}\right)$ be the connected component of this subgraph that contains the vertex $\left(\Upsilon_{0}, e_{G}\right)$.

- On the graph $\left(\left(\oplus_{x \in \mathcal{S}}(\mathbb{Z})_{x}\right) \times \Gamma^{n}, P\right)$, consider the subgraph with vertex set

$$
\left\{(f, \gamma): \operatorname{supp}(f) \in \operatorname{supp}\left(F_{n}\right)\right\}
$$

and edge set made of those original edges of $\left(\left(\oplus_{x \in \mathcal{S}}(\mathbb{Z})_{x}\right) \times \Gamma^{n}, P\right)$ that connects points in the selected vertex set. Let $\Gamma^{n}\left(\Upsilon_{0}\right)$ be the connected component of this subgraph that contains the vertex $\left(\Upsilon_{0}, e_{\Gamma}\right)$.

Next we show that the graphs $\mathcal{G}_{n}\left(\Upsilon_{0}\right)$ and $\boldsymbol{\Gamma}^{n}\left(\Upsilon_{0}\right)$ can be identified. 
Lemma 4.9. Suppose $(G, X, o)$ satisfies $(\Omega)$. Let the subgraphs $\mathcal{G}_{n}\left(\Upsilon_{0}\right)$ and $\Gamma^{n}\left(\Upsilon_{0}\right)$ be defined as above.

(i) The map $\vartheta_{n}$ induces a graph isomorphism

$$
\Theta_{n}: \mathcal{G}_{n}\left(\Upsilon_{0}\right) \rightarrow \Gamma^{n}\left(\Upsilon_{0}\right) .
$$

(ii) For any vertex $g \in \mathcal{G}_{n}\left(\Upsilon_{0}\right), g$ is connected by a labeled edge with an exterior vertex in $\mathcal{G}_{n}\left(\Upsilon_{0}\right)^{c}$ in $(G, Q)$ if and only if $\Theta_{n}(g)$ is connected to $\Gamma^{n}\left(\Upsilon_{0}\right)^{c}$ by an edge with the same label.

Proof. To simplify notation and since as $\Upsilon_{0}$ is fixed, we omit the reference to $\Upsilon_{0}$ in $\mathcal{G}_{n}\left(\Upsilon_{0}\right)$ and $\boldsymbol{\Gamma}^{n}\left(\Upsilon_{0}\right)$.

Define the map $\Theta_{n}$ as follows. First, set $\Theta_{n}\left(\Upsilon_{0}, e_{\Gamma}\right)=\left(\Upsilon_{0}, e_{H}\right)$. For a vertex $(f, \gamma)$ in $\mathcal{G}_{n}$, let $g_{k} \ldots g_{1}$ denote a path inside $\mathcal{G}_{n}$ that starts at $\left(\Upsilon_{0}, e_{\Gamma}\right)$ and ends at $(f, \gamma)$, that is

$$
\begin{gathered}
g_{k} \ldots g_{1}\left(\Upsilon_{0}, e_{\Gamma}\right)=(f, \gamma), \\
g_{j} \ldots g_{1}\left(\Upsilon_{0}, e_{\Gamma}\right) \in \mathcal{G}_{n} \text { for all } 1 \leq j \leq k .
\end{gathered}
$$

Let $\omega$ be the word $\omega=\widehat{g}_{k} \ldots \widehat{g}_{1}$ where $\widehat{g}_{i}=g_{i}$ if $g_{i} \in S$, and $\widehat{g}_{i}=e$ if $g_{i} \in$ $\left\{\left( \pm \mathbf{1}_{1}^{o}, e_{\Gamma}\right)\right\}$, that is generators in $S$ are kept and generators in the lamp direction are removed. With this notation, set

$$
\Theta_{n}(f, \gamma)=\left(f, \vartheta_{n}(\omega)\right) .
$$

Since $g_{k} \ldots g_{1}$ is a path inside $\mathcal{G}_{n}$, Lemma 4.7 implies that

$$
\mathcal{O}\left(g_{k} \ldots g_{1} ; J_{n}\right)=\mathcal{O}\left(\omega ; J_{n}\right) \subseteq \operatorname{supp}(f) \subset B_{n}
$$

Thus $\omega \in \Omega\left(J_{n}, B_{n}\right)$ and, consequently, $\omega$ belongs to the domain of $\vartheta_{n}$. To show that the map $\Theta_{n}$ is well defined, suppose $g_{l}^{\prime} \ldots g_{1}^{\prime}$ is another path inside $\mathcal{G}_{n}\left(\Upsilon_{0}\right)$ that starts at $\left(\Upsilon_{0}, e_{\Gamma}\right)$ and ends at $(f, \gamma)$, then the corresponding $\omega^{\prime}=\widehat{g}_{l}^{\prime} \ldots \widehat{g}_{1}^{\prime}$ is an element in $\Omega\left(J_{n}, B_{n}\right)$ as well, and $\omega$ and $\omega^{\prime}$ have the same evaluation in $\Gamma$. Then Hypothesis $(\Omega)$ implies that $\vartheta_{n}(\omega)=\vartheta_{n}\left(\omega^{\prime}\right)$. That is, $\Theta_{n}(f, \gamma)$ does not depend on the choice of path as desired.

Hypothesis $(\Omega)$ guarantees that $\Theta_{n}$ is injective. To show that $\Theta_{n}$ is surjective, for any vertex $(f, \gamma)$ in $\Gamma^{n}\left(\Upsilon_{0}\right)$, let $p_{l} \ldots p_{1}$ be a path inside $\Gamma^{n}\left(\Upsilon_{0}\right)$ that starts at $\left(\Upsilon_{0}, e_{H}\right)$ and ends at $(f, \gamma)$. If one follows the same path in $(G, Q)$ starting from $\left(\Upsilon_{0}, e_{\Gamma}\right)$, by definition of the two graph structures, the lamp component $\Upsilon_{k}$ along the two paths are exactly the same. Thus the path defined by $p_{l \ldots} p_{1}$ in $(G, Q)$ and starting at $\left(\Upsilon_{0}, e_{\Gamma}\right)$ remains in $\mathcal{G}_{n}\left(\Upsilon_{0}\right)$. It ends at some element $(f, \gamma)$. Then, by the definition of $\Theta_{n}$, we have $\Theta_{n}(f, \gamma)=(f, \gamma)$. This proves that $\Theta_{n}$ is surjective.

By Hypothesis $(\Omega)$, when $\omega_{1}, \omega_{2}, \omega_{1} \omega_{2}$ are all in $\Omega\left(J_{n}, B_{n}\right)$, we have

$$
\vartheta_{n}\left(\omega_{1} \omega_{2}\right)=\vartheta_{n}\left(\omega_{1}\right) \vartheta_{n}\left(\omega_{2}\right)
$$


Hence, if there is a directed edge $\left((f, \gamma),\left(f^{\prime}, \gamma^{\prime}\right)\right)$ in $\mathcal{G}_{n}$, then there is a directed edge $\left(\Theta_{n}(f, \gamma), \Theta_{n}\left(f^{\prime}, \gamma^{\prime}\right)\right)$ in $\boldsymbol{\Gamma}^{n}$ with the same label. Therefore $\Theta_{n}$ is a bijection between $\mathcal{G}_{n}$ and $\boldsymbol{\Gamma}^{n}$ that preserves edge relations. It is a graph isomorphism as desired.

Part (ii) is an immediate consequence of the fact that the lamp component changes in the same way on both graphs $(G, Q)$ and $\left(\left(\oplus_{x \in \mathcal{S}}(\mathbb{Z})_{x}\right) \times \Gamma^{n}, P\right)$ and that the boundaries of $\mathcal{G}_{n}$ and $\Gamma^{n}$ are defined in terms of the lamp component only, without referring to $\gamma$.

\subsection{Test functions}

We now consider transition kernels and test functions on the graphs $(G, Q)$ and $\left(\left(\oplus_{x \in \mathcal{S}}(\mathbb{Z})_{x}\right) \times \Gamma^{n}, P\right)$.

We equip $G=\mathbb{Z} \imath_{\mathcal{S}} \Gamma$ with the right-invariant Markov transition kernel associated with the switch-or-walk measure $\mathfrak{q}=\frac{1}{2}(\eta+\mu)$ where $\eta$ is uniform on $\left( \pm \mathbf{1}_{1}^{o}, e_{\Gamma}\right)$ and $\mu$ is uniform on $S$.

On the graph $\left(\left(\oplus_{x \in \mathcal{S}}(\mathbb{Z})_{x}\right) \times \Gamma^{n}, P\right)$, define a Markov transition kernel $\mathfrak{p}$ as

$$
\mathfrak{p}\left((f, \gamma),\left(f^{\prime}, \gamma^{\prime}\right)\right)=\frac{1}{4} \mathbf{1}_{\left\{f \pm \mathbf{1}_{1}^{\circ}\right\}}\left(f^{\prime}\right)+\frac{1}{2} \sum_{s \in S} \mathbf{1}_{\left\{\vartheta_{n}(s) \gamma\right\}}\left(\gamma^{\prime}\right) \mathbf{1}_{\left.s^{-1} \cdot f\right\}}\left(f^{\prime}\right) \mu(s) .
$$

That is $\mathfrak{p}$ either changes the configuration at $o$ by \pm 1 , or translate $(f, \gamma)$ by a generator $s \in S$. It follows that for $(f, \gamma),\left(f^{\prime}, \gamma^{\prime}\right)$ in $\mathcal{G}_{n}$,

$$
\mathfrak{p}\left(\Theta_{n}(f, \gamma), \Theta_{n}\left(f^{\prime}, \gamma^{\prime}\right)\right)=\mathfrak{q}\left(\left(f^{\prime}, \gamma^{\prime}\right)(f, \gamma)^{-1}\right) .
$$

We now focus on the subgraphs $\mathcal{G}_{n}\left(\Upsilon_{0}\right)$ and $\Gamma^{n}\left(\Upsilon_{0}\right)$ introduced in Notation 4.8 The ingredients we will use to build a test function supported on $\mathcal{G}_{n}\left(\Upsilon_{0}\right)$ include

(a) a $\left(J_{n}, B_{n}\right)$-admissible function $F_{n}$ on $\mathcal{P}_{f}(X)$,

(b) a function $\psi_{n}$ on $\boldsymbol{\Gamma}^{n}$ with finite support.

Given a symmetric probability measure $\mu$ supported on the generating set $S$, we introduce the Rayleigh quotient of the functions $F_{n}$ and $\psi_{n}$ as

$$
\begin{aligned}
\mathcal{Q}_{\mathcal{P}_{f}(\mathcal{S}), \mu}\left(F_{n}\right) & :=\frac{\sum_{s \in \Gamma} \mu(s)\left\|s \cdot F_{n}-F_{n}\right\|_{L^{2}\left(\mathcal{P}_{f}(X)\right)}^{2}}{\left\|F_{n}\right\|_{L^{2}\left(\mathcal{P}_{f}(X)\right)}^{2}} \\
\mathcal{Q}_{\Gamma^{n}, \mu}\left(\psi_{n}\right) & :=\frac{\sum_{s \in \Gamma, x \in \Gamma^{n}} \mu(s)\left|\psi_{n}(x)-\psi_{n}\left(\vartheta_{n}(s) x\right)\right|^{2}}{\left\|\psi_{n}\right\|_{L^{2}\left(\boldsymbol{\Gamma}^{n}\right)}^{2}} .
\end{aligned}
$$

We also set

$$
Q_{\mu}(n):=\mathcal{Q}_{\mathcal{P}_{f}\left(\mathcal{S}_{n}\right), \mu}\left(F_{n}\right)+\mathcal{Q}_{\Gamma^{n}, \mu}\left(\psi_{n}\right) .
$$

Remark 4.10. Assuming that there is a sequence of $\left(J_{n}, B_{n}\right)$-admissible functions $F_{n}$ with $\mathcal{Q}_{\mathcal{P}_{f}(\mathcal{S}), \mu}\left(F_{n}\right)$ tending to zero implies that the action of $\Gamma$ on $\mathcal{S}$ is extensively amenable. Lemma A.4 in the Appendix below provide appropriate test functions $F_{n}$ based on resistance estimates. 
Proposition 4.11. Assume that $(\Gamma, X, o)$ satisfies $(\Omega)$. Let $F_{n}$ be a $\left(J_{n}, B_{n}\right)$ admissible function on $\mathcal{P}_{f}(\mathcal{S})$ and $\psi_{n}$ a function on $\boldsymbol{\Gamma}^{n}$ with finite support. Then for the standard switch-or-walk measure $\mathfrak{q}=\frac{1}{2}(\eta+\mu)$ on $G=\mathbb{Z} \imath_{\mathcal{S}} \Gamma$, we have

$$
\Lambda_{2, G, q}(v) \leq Q_{\mu}(n)
$$

for all $v$ such that

$$
v \geq\left|\vartheta_{n}\left(\Omega\left(J_{n}, B_{n}\right)\right) \cap \operatorname{supp}\left(\psi_{n}\right) h_{0}^{-1}\right| Q_{\mu}(n)^{-\left|B_{n}\right|}
$$

for some $h_{0} \in \operatorname{supp}\left(\psi_{n}\right) \subset \Gamma^{n}$.

Note that

$$
\left|\vartheta_{n}\left(\Omega\left(J_{n}, B_{n}\right)\right) \cap \operatorname{supp}\left(\psi_{n}\right) h_{0}^{-1}\right| \leq \min \left\{\left|\bar{\Omega}\left(J_{n}, B_{n}\right)\right|,\left|\operatorname{supp}\left(\psi_{n}\right)\right|\right\}
$$

and also

$$
\left|\vartheta_{n}\left(\Omega\left(J_{n}, B_{n}\right)\right) \cap \operatorname{supp}\left(\psi_{n}\right) h_{0}^{-1}\right| \leq\left|\bar{\Omega}\left(J_{n}, B_{n}\right) \cap \operatorname{supp}\left(\psi_{n}\right) \operatorname{supp}\left(\psi_{n}\right)^{-1}\right| .
$$

Both upper bounds are independent of $h_{0}$.

Proof. The proof consists of two steps. First we find a test function whose support is contained in the connected component $\Gamma^{n}\left(\Upsilon_{0}\right)$; in the second step the test function is transferred to $\mathcal{G}_{n}\left(\Upsilon_{0}\right)$ via the isomorphism $\Theta_{n}$ of Lemma 4.9

Recall that we are given a $\left(J_{n}, B_{n}\right)$-admissible function $F_{n}$ on on $\mathcal{P}_{f}(\mathcal{S})$ and a function $\psi_{n}$ with finite support on $\Gamma^{n}$. Using this data, we construct a test function $\Phi_{n}$ on $\left(\oplus_{x \in \mathcal{S}}(\mathbb{Z})_{x}\right) \times \Gamma^{n}$ by setting

$$
\Phi_{n}(f, \gamma)=\psi_{n}(\gamma) F_{n}(\operatorname{supp} f) \prod_{x \in \operatorname{Supp} f} \mathbf{1}_{\left[1, Q_{\mu}(n)^{-1}\right]}(f(x)) .
$$

Since $F_{n}$ is $\left(J_{n}, B_{n}\right)$-admissible, there exists a finite set $A_{n}$ such that every set $A$ in the support of $F_{n}$ is of the form $A=g_{A}$. $A_{n}$. It follows that

$$
\left\|\Phi_{n}\right\|_{L^{2}\left(\left(\oplus_{x \in \mathcal{S}}(\mathbb{Z})_{x}\right) \times \Gamma^{n}\right)}^{2}=\left\|\psi_{n}\right\|_{L^{2}\left(\boldsymbol{\Gamma}^{n}\right)}^{2}\left\|F_{n}\right\|_{L^{2}\left(\mathcal{P}_{f}(\mathcal{S})\right)}^{2}\left\lfloor Q_{\mu}(n)^{-1}\right\rfloor^{\left|A_{n}\right|} .
$$

Setting $\left[1, Q_{\mu}(n)^{-1}\right]=I_{n}$, we also note that

$$
\begin{gathered}
\sum_{(f, \gamma)}\left(\psi_{n}(\gamma) F_{n}(\operatorname{supp} f)\right)^{2} \prod_{x \in \operatorname{supp} f} \mathbf{1}_{I_{n}}(f(x)) \mathbf{1}_{I_{n}^{c}}(f(o)+1) \\
=\left\|\psi_{n}\right\|_{L^{2}\left(\Gamma^{n}\right)}^{2}\left\|F_{n}\right\|_{L^{2}\left(\mathcal{P}_{f}(\mathcal{S})\right)}^{2}\left\lfloor Q_{\mu}(n)^{-1}\right\rfloor^{\left|A_{n}\right|-1} .
\end{gathered}
$$

the energy of the function $\Phi_{n}$ with respect to the transition kernel $\mathfrak{p}$ can be 
estimated as

$$
\begin{aligned}
& 2 \mathcal{E}_{\mathfrak{p}}\left(\Phi_{n}, \Phi_{n}\right)=\sum_{(f, h),\left(f^{\prime} h^{\prime}\right)}\left(\Phi_{n}(f, h)-\Phi_{n}\left(f^{\prime}, h^{\prime}\right)\right)^{2} \mathfrak{p}\left((f, h),\left(f^{\prime}, h^{\prime}\right)\right) \\
& \leq \frac{1}{2} \sum_{(f, h)} \sum_{\epsilon= \pm 1}\left(\psi_{n}(h) F_{n}(\operatorname{supp} f)\right)^{2} \prod_{x \in \operatorname{supp} f} \mathbf{1}_{I_{n}}(f(x)) \mathbf{1}_{I_{n}^{c}}(f(o)+\epsilon) \\
& \quad+\sum_{(f, h)} \sum_{s \in S}\left(\psi_{n}(h)-\psi_{n}\left(\vartheta_{n}(s) h\right)\right)^{2} F_{n}(\operatorname{supp} f)^{2} \prod_{x \in \operatorname{supp} f} \mathbf{1}_{I_{n}}(f(x)) \mu(s) \\
& \quad+\sum_{(f, h)} \sum_{s \in S} \psi_{n}(h)^{2}\left(F_{n}(\operatorname{supp} f)-F_{n}(s \cdot \operatorname{supp} f)\right)^{2} \prod_{x \in \operatorname{supp} f} \mathbf{1}_{I_{n}}(f(x)) \mu(s) \\
& \leq 2 Q_{\mu}(n)\left\|\Phi_{n}\right\|_{L^{2}\left(\left(\oplus_{x \in \mathcal{S}}(\mathbb{Z})_{x}\right) \times \Gamma^{n}\right)}^{2}
\end{aligned}
$$

The Markov chain with transition kernel $\mathfrak{p}$ decomposes the space $\left(\oplus_{x \in \mathcal{S}}(\mathbb{Z})_{x}\right) \times$ $\Gamma^{n}$ into connected components. There must exist a $P$-connected subgraph $\mathcal{C}=\mathcal{C}_{n}^{P}$ with vertex set contained in $\operatorname{supp}\left(\Phi_{n}\right)$ such that the restriction of $\Phi_{n}$ to $\mathcal{C}$, denoted by $\Phi_{n}^{\mathcal{C}}$, satisfies

$$
\frac{\mathcal{E}_{\mathfrak{p}}\left(\Phi_{n}^{\mathcal{C}}, \Phi_{n}^{\mathcal{C}}\right)}{\left\|\Phi_{n}^{\mathcal{C}}\right\|_{2}^{2}} \leq \frac{\mathcal{E}_{p}\left(\Phi_{n}, \Phi_{n}\right)}{\left\|\Phi_{n}\right\|_{2}^{2}}
$$

Pick some $\left(f_{0}, h_{0}\right) \in \mathcal{C}$ and translate the $\Gamma^{n}$ component on the right by $h_{0}^{-1}$ by setting

$$
\begin{aligned}
\mathcal{C}_{h_{0}} & =\left\{\left(f, h h_{0}^{-1}\right):(f, h) \in \mathcal{C}\right\}, \\
\Phi_{n, h_{0}}^{\mathcal{C}}(f, h) & = \begin{cases}\Phi_{n}^{\mathcal{C}}\left(f, h h_{0}\right) & \text { if }(f, h) \in \mathcal{C}_{h_{0}} \\
0 & \text { otherwise }\end{cases}
\end{aligned}
$$

If the component $\mathcal{C}$ contains an element of the form $\left(f_{0}, e_{\boldsymbol{\Gamma}^{n}}\right)$, we can pick that point and $\mathcal{C}, \Phi_{n}^{\mathcal{C}}$ stay as they were. In any case, one readily checks that this right translation does not change the Rayleigh quotient of the function, since the transition kernel acts on the left. Since $\operatorname{supp}\left(f_{0}\right) \in \operatorname{supp}\left(F_{n}\right)$, we have obtained a test function $\Phi_{n, h_{0}}^{\mathcal{C}}$ whose support is contained in the subgraph $\Gamma^{n}\left(f_{0}\right)$ given by Notation 4.8. This test function satisfies

$$
\mathcal{E}_{\mathfrak{p}}\left(\Phi_{n, h_{0}}^{\mathcal{C}}, \Phi_{n, h_{0}}^{\mathcal{C}}\right) \leq Q_{\mu}(n)\left\|\Phi_{n, h_{0}}^{\mathcal{C}}\right\|_{L^{2}\left(\left(\oplus_{x \in \mathcal{S}}(\mathbb{Z})_{x}\right) \times \Gamma^{n}\right)}^{2} .
$$

We now proceed with the second step of the proof. Lemma 4.9 describes a graph isomorphism $\Theta_{n}: \mathcal{G}_{n}\left(f_{0}\right) \rightarrow \Gamma^{n}\left(f_{0}\right)$. We can then define a test function $\Psi_{n}$ supported on the subgraph $\mathcal{G}_{n}\left(f_{0}\right)$ in the Cayley graph of $G$ by

$$
\Psi_{n}(f, \gamma)=\Phi_{n, h_{0}}^{\mathcal{C}}\left(\Theta_{n}(f, \gamma)\right) .
$$

Since the domain of $\Phi_{n, h_{0}}^{\mathcal{C}}$ is contained in the range of $\Theta_{n}$, it follows that

$$
\Phi_{n, h_{0}}^{\mathcal{C}}(f, h)= \begin{cases}\Psi_{n}\left(\Theta_{n}^{-1}(f, h)\right) & \text { if }(f, h) \in \Gamma^{n}\left(f_{0}\right) \\ 0 & \text { otherwise. }\end{cases}
$$


Using Lemma 4.9, one checks that indeed

$$
\begin{aligned}
\mathcal{E}_{q}\left(\Psi_{n}, \Psi_{n}\right) & =\mathcal{E}_{p}\left(\Phi_{n, h_{0}}^{\mathcal{C}}, \Phi_{n, h_{0}}^{\mathcal{C}}\right) \\
\left\|\Psi_{n}\right\|_{L^{2}(G)}^{2} & =\left\|\Phi_{n, h_{0}}^{\mathcal{C}}\right\|_{L^{2}\left(\left(\oplus_{x \in \mathcal{S}}(\mathbb{Z})_{x}\right) \times \Gamma^{n}\right)}^{2} .
\end{aligned}
$$

It follows that

$$
\frac{\mathcal{E}_{q}\left(\Psi_{n}, \Psi_{n}\right)}{\left\|\Psi_{n}\right\|_{L^{2}(G)}^{2}} \leq Q_{\mu}(n)
$$

To obtain the estimate on $\Lambda_{2, G, \mathfrak{q}}(v)$ stated in the proposition, it suffices to use an upper bound on the volume of the support of $\Psi_{n}$. By Lemma 4.7, we have

$$
\begin{aligned}
\left|\operatorname{supp}\left(\Psi_{n}\right)\right| & =\left|\operatorname{supp}\left(\Phi_{n, h_{0}}^{\mathcal{C}}\right)\right| \\
& \leq\left|\vartheta_{n}\left(\Omega\left(J_{n}, B_{n}\right)\right) \cap \operatorname{supp}\left(\psi_{n}\right) h_{0}^{-1}\right| Q_{\mu}(n)^{-\left|B_{n}\right|} .
\end{aligned}
$$

Here we also used the fact that, since $F_{n}$ is $\left(J_{n}, B_{n}\right)$-admissible, $\left|A_{n}\right| \leq\left|B_{n}\right|$.

Example 4.12 (The $L^{2}$-isoperimetric profile of $\mathbb{Z} \imath D_{\infty}$ ). To illustrate how the technique described above works in a simple case, we consider the toy example of the infinite dihedral group $D_{\infty}=\left\langle s, t \mid s^{2}=t^{2}=1\right\rangle$ of Example 2.2 Think of it has defined by the Schreier graph of Figure 6. In Example 4.6 we noted that Hypothesis $(\Omega)$ is satisfied with $J_{n}=\{o=0\}, B_{n}=\left\{0, \ldots, 2^{n}-1\right\}$ and $\Gamma^{n}=D_{2^{n}}$. Let $\mu$ be the uniform probability measure on $\{s, t\}$. To construct our test function as in Proposition 4.11 we set $\psi_{n} \equiv 1$ on $\Gamma^{n}$ so that $Q_{\Gamma^{n}, \mu}\left(\psi_{n}\right)=0$.

The function $F_{n}: \mathcal{P}_{f}(\mathcal{S}) \rightarrow \mathbb{R}$ must be $\left(J_{n}, B_{n}\right)$-admissible which means it is supported on subsets $Y$ of the form $Y=g \cdot A_{n}$ with $o \in Y \subset B_{n}$ for some $A_{n}$. Let

$$
A_{n}=\left\{0, \ldots, 2^{n-1}-1\right\} .
$$

Define $F_{n}$ so that $F_{n}(Y)=0$ unless $Y=g \cdot A_{n}$ where $g$ is a reduced word in $s, t$ of length $\ell \leq 2^{n-1}$ which terminates with $t$, in which case

$$
F_{n}(Y)=1-2^{-n+1} l \text {. }
$$

We need to verify that $F_{n}$ is $\left(J_{n}, B_{n}\right)$-admissible. This follows by inspection because $g \cdot A_{n}$ is

$$
\left\{0, \ldots, 2^{n-1}-1-l\right\} \cup\left\{2^{n-1}-l-1+2 k: 1 \leq k \leq l\right\}
$$

when $0 \leq l \leq 2^{n-1}-1$ and $\{2 k-1: 1 \leq k \leq l\}$ when $l=2^{n-1}$.

The Rayleigh quotient of $F_{n}$ can be computed

$$
\mathcal{Q}_{\mathcal{P}_{f}(\mathcal{S}), \mu}\left(F_{n}\right) \sim \frac{3}{\left(2^{n-1}\right)^{2}} .
$$

To apply Proposition 4.11 observe that $\left|\bar{\Omega}\left(J_{n}, B_{n}\right)\right|,\left|\operatorname{supp}\left(\psi_{n}\right)\right|$ and $\left|B_{n}\right|$ are all equal to $2^{n}$. This yields

$$
\Lambda_{2, \mathbb{Z} \imath_{\mathcal{S}} D_{\infty}, q}(v) \leq \frac{C}{\left(2^{n}\right)^{2}}, \text { for any } v \geq C^{-1} 2^{n 2^{n} / C},
$$


that is (with a different $C$ ),

$$
\Lambda_{2, \mathbb{Z} \imath_{\mathcal{S}} D_{\infty}, q}(v) \lesssim\left(\frac{\log \log v}{\log v}\right)^{2} .
$$

This gives the return probability lower bound

$$
\Phi_{\mathbb{Z} \imath_{\mathcal{S}} D_{\infty}}(n) \gtrsim \exp \left(-n^{\frac{1}{3}} \log ^{\frac{2}{3}} n\right) .
$$

By Example 3.9 and the Cheeger inequality (2.2), have a matching lower bound for $\Lambda_{2, \mathbb{Z} \imath_{S} D_{\infty}, q}$ so that

$$
\Lambda_{2, \mathbb{Z} \imath_{\mathcal{S}} D_{\infty}, q}(v) \simeq\left(\frac{\log \log v}{\log v}\right)^{2} .
$$

and

$$
\Phi_{\mathbb{Z}_{\mathcal{S}} D_{\infty}}(n) \simeq \exp \left(-n^{\frac{1}{3}} \log ^{\frac{2}{3}} n\right) .
$$

\section{$5 \quad$ Bubble groups}

This section is devoted to a family of groups considered in [21] where the name "bubble group" is used. The marked Schreier graph pictured in Figure 8 defines a finite bubble group.

Figure 8: The marked Schreier graph of the finite bubble group with $\mathbf{a}=$ $(2,5,9), \mathbf{b}=(3,3)$. The generators $a, b$ each acts clockwise along its respective cycle. Each vertex of degree 2 carries a self-loop marked $b$ which is not shown except at the root.

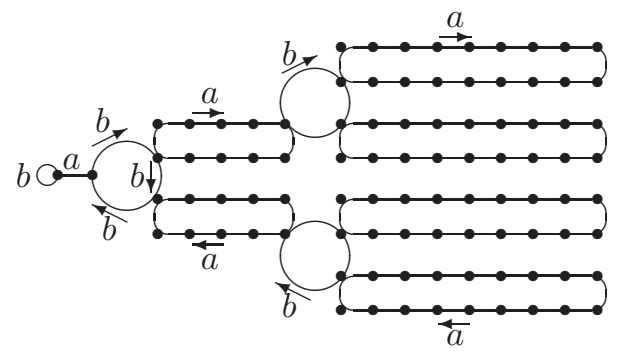

\subsection{The general bubble group}

Let $\mathbf{a}=\left(a_{1}, a_{2}, \ldots\right)$ and $\mathbf{b}=\left(b_{1}, b_{2}, ..\right)$ be two natural integer sequences (finite or infinite; if the sequence are finite, $\mathbf{b}$ is one element shorter than $\mathbf{a})$. The "bubble group" $\Gamma_{\mathbf{a}, \mathbf{b}}$ is associated with the tree like bubble graph $X_{\mathbf{a}, \mathbf{b}}$ were $X_{\mathbf{a}, \mathbf{b}}$ is obtained from the rooted tree $\mathbf{T}_{\mathbf{b}}$ with forward degree sequence (1, $b_{1}-$ 
Figure 9: A piece of the marked Schreier graph of an infinite bubble group with $\mathbf{a}=\left(a_{1}, a_{2}, \ldots\right), \mathbf{b}=(3,3,3 \ldots)$.

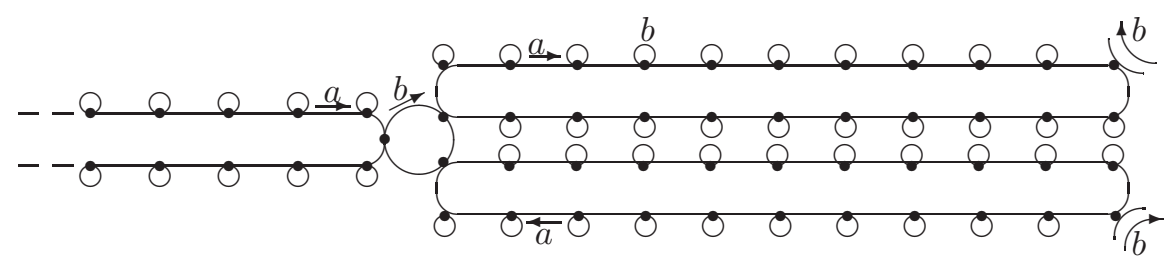

$1, b_{2}-1, \ldots$ ) as follows. Each edge at level $k \geq 1$ in the tree (we make the convention that the level of an edge is the level of the child on that edge) is replaced by a cycle of length $2 a_{k}$ called a bubble. Each vertex at level $k \geq 1$ (we ignore the root which is now part of a circle of length $2 a_{1}$ ) is blown-up to a $b_{k}$-cycle with each vertex of this cycle inheriting one of the associated $2 a_{k+1^{-}}$ cycle. These $b_{k}$-cycles are called branching cycles. Finally, at each vertex which belong only to a bubble (but not to a branching cycle), we add a self loop. The vertex set of the graph $X_{\mathbf{a}, \mathbf{b}}$ can be parametrized using pairs $(w, u)$ with $w$ a finite word in

$$
\{\emptyset\} \cup\left(\cup_{k=1}^{\infty}\left\{1, \ldots, b_{1}-1\right\} \times\left\{1, \ldots, b_{2}-1\right\} \times \cdots \times\left\{1, \ldots, b_{k}-1\right\}\right)
$$

and $u \in\left\{0, \ldots, 2 a_{k+1}-1\right\}$ if $|w|=k$. By definition, the vertex $o=\emptyset$ is the root.

In the graph $X_{\mathbf{a}, \mathbf{b}}$, we call "level k" the set of all the vertices $(w, u)$ with $|w|=k-1,0 \leq u \leq 2 a_{k}-1$. If all the $a_{k}$ are distinct, this is the set of all vertices that belong to a bubble of length $2 a_{k}$. We say that a branching cycle is at "level k" if it is attached at the far end (i.e., furthest away from $o$ ) of a level- $k$ bubble. Note that the vertices of any branching cycle at level $k$ are parametrized as follows:

- $\left(w^{\prime}, a_{k}\right)$ with $\left|w^{\prime}\right|=k-1$ for the vertex closest to the root $o$, a vertex which also belongs to a level- $k$ bubble,

- $\left(w^{\prime} z, 0\right)$ with $z \in\left\{1, \ldots, b_{k}-1\right\}$ for the other vertices on that branching cycle, each of which also belongs to a level- $(k+1)$ bubble.

We let

$$
\mathfrak{b}\left(w^{\prime}\right)=\left\{\left(w^{\prime}, a_{k}\right),\left(w^{\prime} 1,0\right), \ldots,\left(w^{\prime}\left(b_{k}-1\right), 0\right)\right\}
$$

denote the branching cycle at $\left(w^{\prime}, a_{k}\right)$.

Having chosen an orientation along each cycle (say, clockwise), we label each edge of the bubble with the letter $a$ and each edge of the branching cycle with the letter $b$. 
The group $\Gamma_{\mathbf{a}, \mathbf{b}}$ is a subgroup of the (full) permutation group of the vertex set of $X_{\mathbf{a}, \mathbf{b}}$ generated by two elements $\alpha$ and $\beta$. Informally, $\alpha$ rotates the bubbles whereas $\beta$ rotates the branching cycles. Formally, the action of the permutation $\alpha$ (resp. $\beta$ ) on any vertex $x$ in $X_{\mathbf{a}, \mathbf{b}}$ is indicated by the oriented labeled edge at $x$ marked with an $a$ (resp. a $b$ ). Obviously, we can replace the edge labels $a, b$ with the group elements $\alpha, \beta$, once these are defined.

Lemma 5.1. For any choice of the sequence $\mathbf{a}, \mathbf{b}$ with $b_{i} \geq 3$ for all $i$, the group $\Gamma_{\mathbf{a}, \mathbf{b}}$ has exponential volume growth.

Remark 5.2. If $\mathbf{a}, \mathbf{b}$ are constant sequences with $a_{i}=a$ and $b_{i}=2$ for all $i$ then the group $\Gamma_{\mathbf{a}, \mathbf{2}}$ actually falls into the class of Neumann-Segal type groups discussed in Section [6. In particular, it is a subgroup of the automorphism group of the tree $\mathbb{T}_{\bar{d}}$ with $\bar{d}=\left(d_{i}\right)_{1}^{\infty}, d_{1}=2 a, d_{i}=2, i \geq 2$. This description of the group $\Gamma_{\mathbf{a}, \mathbf{2}}$ shows that it is a subgroup of the group $D_{\infty} \prec(\mathbb{Z} / 2 a \mathbb{Z})$. Hence $\Gamma_{\mathbf{a}, 2}$ has polynomial volume growth of degree at most $2 a$.

Proof. If $\mathbf{a}$ is bounded and all $b_{i} \geq 3$, it is obvious that $\Gamma_{\mathbf{a}, \mathbf{b}}$ has exponential volume growth. If $\mathbf{a}$ is unbounded we show that the words

$$
\prod_{1}^{n} \alpha^{n-i} \beta^{\epsilon_{i}} \alpha^{n-i}=\alpha^{n-1} \beta^{\epsilon_{1}} \alpha^{-1} \beta^{\epsilon_{2}} \alpha^{-1} \ldots \beta^{\epsilon_{n-1}}, \epsilon_{i} \in\{ \pm 1\}
$$

are all distinct. We proceed by induction on $n$. If $n=k=1$ the property is obviously satisfied. Assume $k \geq 1$ and the property is true for all $n \leq k$. Pick to elements $g, g$ of the form above with $n=k+1$ with respective sequence $\left(\epsilon_{j}\right)_{1}^{n},\left(\epsilon_{j}^{\prime}\right)_{1}^{n}$. Since $a_{i}$ is unbounded, we can find $i$ such that $a_{i} \geq k+2$. Let $w=1 \ldots 1$ of length $|w|=i-1, u=k+1$, and consider the point $x=(w, u)$ on the Schreier graph $X_{\mathbf{a}, \mathbf{b}}$. If $\epsilon_{n} \neq \epsilon_{n}^{\prime}$, direct inspection shows that the elements $g, g^{\prime}$ move $x$ to two different bubbles, one at level $i-1$ and the other at level $i$. If $\epsilon_{n}=\epsilon_{n}^{\prime}$, apply the induction hypothesis to

$$
\prod_{2}^{n} \alpha^{n-i} \beta^{\epsilon_{i}} \alpha^{n-i}, \prod_{2}^{n} \alpha^{n-i} \beta^{\epsilon_{i}^{\prime}} \alpha^{n-i} .
$$

This concludes the proof.

We also consider the associated finite bubble groups $\Gamma_{\mathbf{a}, \mathbf{b}}^{k}$ defined by action on truncated bubble graphs. Let $X_{\mathbf{a}, \mathbf{b}}^{k}$ denote the first $k$ levels of the bubble graph $X_{\mathbf{a}, \mathbf{b}}$. The finite bubble group $\Gamma_{\mathbf{a}, \mathbf{b}}^{k}$ is generated by $\alpha_{k}$ and $\beta_{k}$, where $\alpha_{k}$ acts in the same way as $\alpha$ by rotating the long bubbles, and $\beta_{k}$ acts in the same way as $\beta$ except that, at level $k, \beta_{k}$ stabilizes the end points instead of moving them along the branching cycle. See Figure 8 .

\subsection{Local embedding under Assumption (A)}

Definition 5.3 (Assumption (A)). We say that assumption (A) is satisfied if the sequence $\mathbf{b}=\left(b_{1}, b_{2}, \ldots\right)$ is constant (i.e., $b_{i}=b$ for all $\left.i=1, \ldots\right)$ and the 
scaling sequence $\mathbf{a}=\left(a_{1}, a_{2}, \ldots\right)$ is monotone increasing to $\infty$, that is

$$
1 \leq a_{1} \leq a_{2} \leq \ldots, \lim _{n \rightarrow \infty} a_{n}=\infty
$$

Without the assumption that the sequence $a_{n}$ increases to infinity, the group $\Gamma_{\mathbf{a}, \mathbf{b}}$ may be non-amenable. For instance, consider the case when a is the constant sequence $a_{i}=2$ and $b=3$. Then $\Gamma_{\mathbf{a}, \mathbf{b}}=\mathbb{Z}_{2} * \mathbb{Z}_{3}$. More generally, $\Gamma_{\mathbf{a}, \mathbf{b}}$ is non-amenable whenever both sequences $\mathbf{a}, \mathbf{b}$ are bounded. For an arbitrary sequence $\mathbf{b}$, the condition $\lim \inf a_{i}=\infty$ suffices to imply that $\Gamma_{\mathbf{a}, \mathbf{b}}$ is amenable. See Section 5.5.

The method of this section still applies if we modify Assumption(A) by replacing the hypothesis that $\mathbf{b}$ is constant by the requirement that it is bounded (hence, take only finitely many values). The length of the intervals between different occurrences of a complete collection of the values taken by $\mathbf{b}$ play a key role in the form of the estimates that can be produced by this method. In the case of periodic (or quasi-periodic) b, the estimates are the same as in the case of a constant sequence $\mathbf{b}$ but if a certain value, say 5 , appears only along a very sparse sequence of indices then the estimates deteriorate. See Section 5.5 .

In this section, we work under Assumption (A). We drop the explicit references to the sequences $\mathbf{a}, \mathbf{b}$ and write

$$
\Gamma_{\mathbf{a}, \mathbf{b}}=\Gamma, \quad X_{\mathbf{a}, \mathbf{b}}=X
$$

Set

$$
\mathfrak{N}(w, r)=\{x \in X: d(x, \mathfrak{b}(w)) \leq r\}, w \in\{0, \ldots, b-1\}^{(\infty)}, r>0
$$

For any $k \leq j, w$ of length $|w|=j$ and $0 \leq r \leq a_{k-1}-1$, we have an obvious bijective map

$$
\iota_{k}^{w}: \mathfrak{N}(w, r) \mapsto \mathfrak{N}\left(1^{k-1}, r\right)
$$

which can be used to identify these vertex sets.

In order to fit the bubble group $\Gamma$ into the general framework introduced in Section 4, we need to explain how to choose the sets $J_{k}, B_{k}$ and the approximation groups $\Gamma^{k}$ so that the associated local embeddings $\vartheta_{k}$ required for Hypothesis $(\Omega)$ can be proved to exists. See Definition 4.4.

Let $\mathbf{S}$ be the alphabet $\mathbf{S}=\left\{\alpha^{ \pm 1}, \beta^{ \pm 1}\right\}$. Given subsets $J, B$ of $X$ with $J \subset B$, recall that the set $\Omega(J, B) \subset \mathbf{S}^{(\infty)}$ is defined as

$$
\Omega(J, B)=\left\{\omega \in \mathbf{S}^{(\infty)}: \mathcal{O}(\omega, J) \subset B\right\} .
$$

For a given level $k$, we set

$$
\mathfrak{m}_{k}=\left(1^{k-1}, a_{k} / 2\right), \quad J_{k}=\left\{\mathfrak{m}_{k}\right\}
$$

and

$$
B_{k}(l)=\left\{x \in X: d\left(x, \mathfrak{m}_{k}\right) \leq l\right\}, 0 \leq l \leq\left(a_{k} / 2\right)-1 .
$$


Figure 10: Sketch of the Schreier graph $X$ : levels, $\mathfrak{b}(w), \mathfrak{m}_{k}$. Details of the red circle region are shown in Figure 11

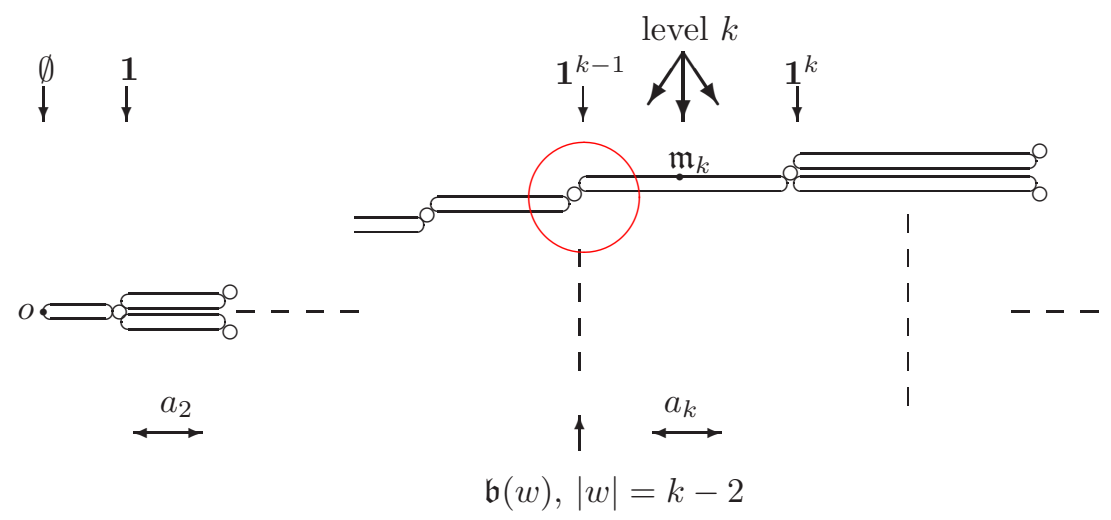

Lemma 5.4. Fix $0 \leq t \leq\left(a_{k} / 2\right)-1$. Let $\omega=\gamma_{1} \ldots \gamma_{q}$ be a word of length $q$ such that $\gamma_{j} \ldots \gamma_{q} \cdot \mathfrak{m}_{k} \in B_{k}(t)$ for each $1 \leq j \leq q$. Then there exists $s_{\omega}$ such that

$$
\gamma_{1} \ldots \gamma_{q} \cdot \mathfrak{m}_{k}=\alpha^{s_{\omega}} \cdot \mathfrak{m}_{k} \text { and } \gamma_{1}^{-1} \ldots \gamma_{q}^{-1} \cdot \mathfrak{m}_{k}=\alpha^{-s_{\omega}} \cdot \mathfrak{m}_{k} .
$$

Further, let $s$ be an integer such that $|s|+t \leq\left(a_{k} / 2\right)-1$ and set $x=\alpha^{s} \mathfrak{m}_{k}$. Then

$$
\alpha^{s} \omega \cdot \mathfrak{m}_{k}=\omega \alpha^{s} \cdot \mathfrak{m}_{k}=\omega \cdot x .
$$

In particular, we have

$$
d\left(\omega \cdot \mathfrak{m}_{k}, \omega \cdot x\right)=d\left(\mathfrak{m}_{k}, x\right) .
$$

Proof. Writing $\mathfrak{m}=\mathfrak{m}_{k}$, the first assertion is easily proved by considering $\gamma_{j} \ldots \gamma_{q} \cdot \mathfrak{m}$ and by descending induction on $j \leq q$.

The second assertion is also proved by descending induction on $j \leq q$. The statement is obvious for $j=q$. Assume the claim is true for $j+1$. By hypothesis, $d\left(\gamma_{j+1} \ldots \gamma_{q} \cdot \mathfrak{m}, \mathfrak{m}\right) \leq t$. Since $|s|+t \leq\left(a_{k} / 2\right)-1$, it follows that the action of $\alpha^{s} \gamma_{j}$ on $\gamma_{j+1} \ldots \gamma_{q} \cdot \mathfrak{m}$ is the same as the action of $\gamma_{j} \alpha^{s}$. Hence

$$
\alpha^{s} \cdot\left(\gamma_{j} \ldots \gamma_{q} \cdot \mathfrak{m}\right)=\gamma_{j} \alpha^{s} \gamma_{j+1} \ldots \gamma_{q} \cdot \mathfrak{m}=\gamma_{j} \ldots \gamma_{q} \alpha^{s} \cdot \mathfrak{m} .
$$

Lemma 5.5. Assume that $\omega=\gamma_{1} \ldots \gamma_{p} \in \Omega_{k}(l)=\Omega\left(\mathfrak{m}_{k}, B_{k}(l)\right)$ with $l \leq$ $\left(a_{k} / 4\right)-1$. Then, for any $1 \leq j \leq p$, we have $\gamma_{1} \ldots \gamma_{j}=\alpha^{s_{j}} \mathfrak{m}_{k}$ with $\left|s_{j}\right| \leq l$ and

$$
\gamma_{1} \ldots \gamma_{j-1} \gamma_{j} \cdot \mathfrak{m}_{k}=\gamma_{j} \gamma_{j-1} \ldots \gamma_{1} \cdot \mathfrak{m}_{k} .
$$

Further, for every subword $u=\gamma_{i} \ldots \gamma_{j}$ of $w$,

$$
u \cdot \mathfrak{m}_{k}=\alpha^{s_{j}-s_{i-1}} \cdot \mathfrak{m}_{k} \text { and } u^{-1} \cdot \mathfrak{m}_{k}=\alpha^{-s_{j}+s_{i-1}} \cdot \mathfrak{m}_{k} .
$$


Figure 11: Sketch showing $\mathfrak{N}\left(\mathbf{1}^{k-2}, r\right), \mathfrak{m}_{k}$

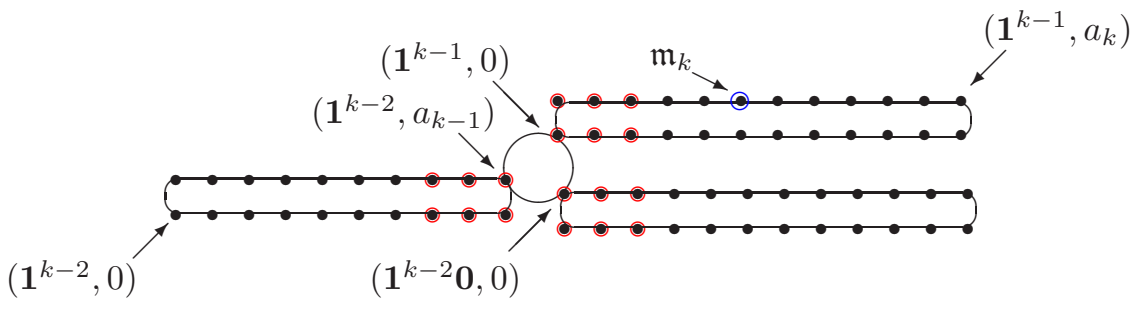

Proof. We set $\mathfrak{m}=\mathfrak{m}_{k}$. The first statement is proved by induction on the length $p$ of the word $\omega$. The desired property is obviously true for $p=1$. Assume that it has been proved for all words of length at most $p$.

Consider $\omega=\gamma_{1} \ldots \gamma_{p+1} \in \Omega_{k}(l)$ and write

$$
\gamma_{1} \ldots \gamma_{p+1} \cdot \mathfrak{m}=\left(\gamma_{1} \ldots \gamma_{p}\right) \cdot\left(\gamma_{p+1} \cdot \mathfrak{m}\right) .
$$

By hypothesis, $\gamma_{1} \cdot \mathfrak{m}, \gamma_{1} \gamma_{2} \cdot \mathfrak{m}, \ldots, \gamma_{1} \ldots \gamma_{p} \cdot \mathfrak{m}$ are in $B(l)$. By the induction hypothesis, $\gamma_{i} \ldots \gamma_{p} \cdot \mathfrak{m}=\alpha^{s_{p}-s_{i-1}} \cdot \mathfrak{m}, 1 \leq i \leq p$. Hence Lemma 5.4 and the induction hypothesis give

$$
\begin{aligned}
\gamma_{1} \ldots \gamma_{p} \gamma_{p+1} \cdot \mathfrak{m} & =\gamma_{p+1} \cdot\left(\gamma_{1} \ldots \gamma_{p} \cdot \mathfrak{m}\right) \\
& =\gamma_{p+1} \cdot\left(\gamma_{p} \ldots \gamma_{1} \cdot \mathfrak{m}\right) \\
& =\gamma_{p+1} \gamma_{p} \ldots \gamma_{1} \cdot \mathfrak{m} .
\end{aligned}
$$

We need to show that, for any subword $\omega^{\prime}=\gamma_{j} \ldots \gamma_{p+1}$,

$$
\omega^{\prime} \cdot \mathfrak{m}=\alpha^{s_{p+1}-s_{j-1}} \cdot \mathfrak{m} \text { and }\left(\omega^{\prime}\right)^{-1} \cdot \mathfrak{m}=\alpha^{-s_{p+1}+s_{j-1}} \cdot \mathfrak{m}
$$

We write

$$
\begin{aligned}
\omega^{\prime} \cdot \mathfrak{m} & =\left(\gamma_{1} \ldots \gamma_{j-1}\right)^{-1} \gamma_{1} \ldots \gamma_{p+1} \cdot \mathfrak{m} \\
& =\left(\gamma_{1} \ldots \gamma_{j-1}\right)^{-1} \alpha^{s_{p+1}} \cdot \mathfrak{m}
\end{aligned}
$$

and

$$
\begin{aligned}
\left(\omega^{\prime}\right)^{-1} \cdot \mathfrak{m} & =\gamma_{p+1}^{-1} \ldots \gamma_{1}^{-1}\left(\gamma_{1} \ldots \gamma_{j-1}\right) \cdot \mathfrak{m} \\
& =\gamma_{p+1}^{-1} \ldots \gamma_{1}^{-1} \alpha^{s_{j-1}} \cdot \mathfrak{m} .
\end{aligned}
$$

We have proved that $\gamma_{i} \ldots \gamma_{1} \cdot \mathfrak{m} \in B(l)$ for each $1 \leq i \leq p+1$. Lemma 5.4 shows that $\gamma_{i}^{-1} \ldots \gamma_{1}^{-1} \cdot \mathfrak{m} \in B(l)$. By the second part of Lemma [5.4, we have

$$
\omega^{\prime} \cdot \mathfrak{m}=\left(\gamma_{1} \ldots \gamma_{j-1}\right)^{-1} \alpha^{s_{p+1}} \cdot \mathfrak{m}=\alpha^{s_{p+1}} \alpha^{-s_{j-1}} \cdot \mathfrak{m} .
$$


and

$$
\left(\omega^{\prime}\right)^{-1} \cdot \mathfrak{m}=\left(\gamma_{p+1}^{-1} \ldots \gamma_{1}^{-1}\right) \alpha^{s_{j-1}} \cdot \mathfrak{m}=\alpha^{s_{j-1}} \alpha^{-s_{p+1}} \cdot \mathfrak{m} .
$$

The following observation regarding repetition of orbits is straightforward. Given a word $w=w_{1} \ldots w_{p}$, consider the orbit $\left\{w_{s}^{-1} \ldots w_{1}^{-1} \cdot x\right\}_{1 \leq s \leq p}$ of $x$. Note that this can be thought of as a forward orbit, it behaves very differently from the inverted orbit $\mathcal{O}(w ; x)$.

Lemma 5.6 (Identification of orbits). Fix $k \geq 0$ and $0 \leq l \leq\left(a_{k} / 4\right)-1$. For any $t \geq k+1$ and any vertex $x=(w, u)$ with $|w|=t-1$ and $0 \leq u \leq 2 a_{t}-1$ (i.e., any vertex at level $t$ ), let $w^{\prime}$ be the parent of $w$ and set

$$
\hat{x}=\left\{\begin{array}{cll}
\mathfrak{m}_{k} & \text { if } \quad d\left(x,\left\{(w, 0),\left(w, a_{t}\right)\right)\right)>l, \\
\iota_{k}^{w^{\prime}}(x) & \text { if } \quad d(x,(w, 0)) \leq l, \\
\iota_{k}^{w}(x) & \text { if } \quad d\left(x,\left(w, a_{t}\right)\right) \leq l .
\end{array}\right.
$$

In the first case, define $\hat{\iota}=\hat{\iota}_{x}$ to be the obvious map taking the segment $\{(w, v)$ : $|v-u| \leq l\}$ to the segment $\left.\left\{\left(1^{k-1}, v\right)\right):\left|v-\left(a_{k} / 2\right)\right| \leq l\right\}$. In the second and third cases, set $\hat{\imath}=\iota_{k}^{w^{\prime}}$ and $\hat{\iota}=\iota_{k}^{w}$, respectively. Let $\omega=\gamma_{1} \ldots \gamma_{p}$ be a word that belongs to $\Omega_{k}(l)$. Then, for any $1 \leq j \leq p$,

$$
\hat{\iota}_{x}\left(\gamma_{j}^{-1} \ldots \gamma_{1}^{-1} \cdot x\right)=\gamma_{j}^{-1} \ldots \gamma_{1}^{-1} \cdot \hat{x} .
$$

Proof. As before, we write $\mathfrak{m}=\mathfrak{m}_{k}$. For a point $x$ at distance $>l$ from any branching cycle, we show that the orbit $\left\{w_{s}^{-1} \ldots w_{1}^{-1} \cdot x\right\}_{1 \leq s \leq p}$ up to time $p$ is contained in $B(x, l)$. If not, let $s$ be the first time that $d\left(w_{s}^{-1} \ldots w_{1}^{-1} \cdot x, x\right)=$ $l+1$. Since, up to time $s$, the orbit must remain within in the bubble containing $x$, it can be identified (using $\hat{i}$ ) with $\left\{w_{j}^{-1} \ldots w_{1}^{-1} \cdot \mathfrak{m}\right\}_{1 \leq j \leq s}$. It follows that $d\left(w_{s}^{-1} \ldots w_{1}^{-1} \cdot \mathfrak{m}, \mathfrak{m}\right)=l+1$. By lemma 5.5, we have $d\left(\mathfrak{m}, w_{1} \ldots w_{s} \cdot \mathfrak{m}\right)=l+1$. This contradicts the assumption that $w \in \Omega(\mathfrak{m}, B(\mathfrak{m}, l))$. Therefore the whole orbit up to time $p$ is in $B(x, l)$.

Next, let $x=(w, u)$ be within distance $l$ from one of the branching cycle, say $d(x,(w, 0)) \leq l$ (the other case is treated in the same way). We claim that the orbit $\left\{w_{s}^{-1} \ldots w_{1}^{-1} \cdot x\right\}_{1 \leq s \leq p}$ cannot leave the set

$$
\mathfrak{N}\left(w^{\prime}, 3 l+1\right)=\left\{z: d\left(z, \mathfrak{b}\left(w^{\prime}\right)\right) \leq 3 l+1\right\} .
$$

Suppose on the contrary that the orbit exits $\mathfrak{N}\left(w^{\prime}, 3 l+1\right)$. Let $t \leq p$ be the first time such that $d\left(w_{t}^{-1} \ldots w_{1}^{-1} \cdot x, \mathfrak{b}\left(w^{\prime}\right)\right)=3 l+2$. Let $y=w_{t}^{-1} \ldots w_{1}^{-1} \cdot x$, since the orbits starts at $x$ and $t$ is the first time $d\left(w_{t}^{-1} \ldots w_{1}^{-1} \cdot x, \mathfrak{b}\left(w^{\prime}\right)\right)=3 l+2$, there must be a largest time $s \leq t$ such that $d\left(w_{s}^{-1} \ldots w_{1}^{-1} \cdot x, \mathfrak{b}\left(w^{\prime}\right)\right)=l+1$ and $d\left(w_{s}^{-1} \ldots w_{1}^{-1} \cdot x, y\right)=2 l+1$. Set $z=\gamma_{s}^{-1} \ldots \gamma_{1}^{-1} \cdot x$. By definition of $s$ and $t, z$ orbit

$$
\left\{\gamma_{s+i}^{-1} \ldots \gamma_{s+1}^{-1} \cdot z: 0 \leq i \leq t-s\right\}
$$


remains in the segment between $y$ and $z$ and we must have

$$
2 l+1=d\left(z, \gamma_{t}^{-1} \cdots \gamma_{s+1}^{-1} \cdot z\right)=d\left(\mathfrak{m}, \gamma_{t}^{-1} \cdots \gamma_{s+1}^{-1} \cdot \mathfrak{m}\right),
$$

By Lemma 5.5, this contradicts the assumption that $\omega \in \Omega_{k}(l)$.

Recall that $\mathbf{S}=\left\{\alpha^{ \pm 1}, \beta^{ \pm 1}\right\}$ and that we have the evaluation map

$$
\theta: \mathbf{S}^{(\infty)} \rightarrow \Gamma_{\mathbf{a}, \mathbf{b}}, \quad \theta_{k}: \mathbf{S}^{(\infty)} \rightarrow \Gamma_{\mathbf{a}, \mathbf{b}}^{k}
$$

Lemma 5.7 (Local embeddings). Fix $k \geq 0$. Let $l$ be an integer such that $0 \leq l<\left(a_{k} / 4\right)-1$. The restriction of the map $\vartheta_{k}=\theta_{k+1}: \mathbf{S}^{(\infty)} \rightarrow \mathbf{G}^{k}=\Gamma_{\mathbf{a}, \mathbf{b}}^{k+1}$ to the set $\Omega_{k}(l)$ satisfies the conditions in part 2 of Hypothesis $(\Omega)$.

Proof. Let $\theta: \mathbf{S}^{(\infty)}$ be the evaluation map in $\Gamma$. By the definition of $\vartheta_{k}$, it remains to show that for any $w, w^{\prime} \in \Omega_{k}(l), \theta(w)=\theta(w)$ if and only if $\vartheta_{k}(w)=$ $\vartheta_{k}\left(w^{\prime}\right)$. In other words, setting $\operatorname{ker}\left(\vartheta_{k}\right)=\left\{\omega \in \mathbf{S}^{(\infty)}: \vartheta_{k}(\omega)=e\right\}$, we need to show

$$
\operatorname{ker}\left(\vartheta_{k}\right) \cap \Omega_{k}(l)=\operatorname{ker}(\theta) \cap \Omega_{k}(l) .
$$

We first show that

$$
\operatorname{ker}\left(\vartheta_{k}\right) \cap \Omega_{k}(l) \subset \operatorname{ker}(\theta) \cap \Omega_{k}(l)
$$

Suppose there exists $\omega \in \operatorname{ker}\left(\vartheta_{k}\right) \cap \Omega_{k}(l)$ such that $\omega \neq e_{\Gamma}$ in $\Gamma$. Then there exists some point $x \in X$ such that $\omega \cdot x \neq x$. If $x=(w, u)$ with $|w| \leq k$, we set $\hat{x}=x$. Otherwise, $\hat{x}$ is given by Lemma 5.6

From Lemma 5.6. we know that $\hat{x}$ is also moved by $\omega$. By Lemma 5.6 the constraint $\omega \in \Omega_{k}(l)$ implies that the orbit $\left\{\gamma_{j}^{-1} \ldots \gamma_{1}^{-1} \cdot \widehat{x}\right\}_{1 \leq j \leq p}$ never touches any point of the form $\left(w, a_{k+1}\right)$ with $|w|=k$ (that is, any of the end points of level $k+1$ ). Therefore the orbit of $\hat{x}$ in $X$ is exactly the same as the orbit in the finite bubble graph $X^{k+1}$. In particular, they will end at the same place, so that $\vartheta_{k}(\omega) \cdot \hat{x} \neq \hat{x}$. This obviously contradicts the assumption that $\vartheta_{k}(\omega)$ is trivial.

In the other direction, we assume that there exists $\omega \in \operatorname{ker}(\theta) \cap \Omega_{k}(l)$ such that $\vartheta_{k}(\omega)$ is non-trivial. In such case, there exists a point $x=(w, u) \in X^{k+1}$, $|w| \leq k$, such that $\vartheta_{k}(\omega) \cdot x \neq x$. If $|w| \leq k-1$ or $|w|=k$ and $d\left(x,\left(w, a_{k+1}\right)\right)>l$ then (following the same line of reasoning as before) the orbit

$$
\left\{\theta_{k}\left(\gamma_{j}^{-1} \ldots \gamma_{1}^{-1}\right) \cdot x\right\}_{1 \leq j \leq p}
$$

in $X^{k+1}$ can readily be identified with the orbit of $(w, u)$ in $X$. This gives a contradiction. If $|w|=k$ and $d\left(x,\left(w, a_{k}\right)\right) \leq l$, then the condition $\omega \in \Omega_{k}(l)$ implies that the orbit $\left\{\pi\left(\gamma_{j}^{-1} \ldots \gamma_{1}^{-1}\right) \cdot(w, u)\right\}_{1 \leq j \leq p}$ in $X$ stays in $\mathfrak{N}(w, 3 l+1)$. Since $\pi\left(\gamma_{p}^{-1} \ldots \gamma_{1}^{-1}\right) \cdot(w, u)=(w, u)$ in $X$, it now follows by inspection that $\vartheta_{k}\left(\gamma_{p}^{-1} \ldots \gamma_{1}^{-1}\right) \cdot x=x$ in $X^{k+1}$. This is the desired contradiction. 


\subsection{Isoperimetric profiles of bubble groups}

In this section, we prove and illustrate the following Theorem.

Theorem 5.8. Let $\Gamma=\Gamma_{\mathbf{a}, \mathbf{b}}$ be a bubble group with scaling sequence a and branching sequence $\mathbf{b}$ that satisfy Assumption (A). Let $G=\mathbb{Z} \imath_{X} \Gamma$ be the associated permutation wreath product. There exist a constant $C$ such that for all $r>1$,

$$
\Lambda_{1, G}(v) \geq \frac{1}{C r} \text { for all } v \leq C^{-1} r^{\left|B_{X}(o, r)\right| / C} .
$$

Furthermore, we also have

$$
\Lambda_{2, G}(v) \leq \frac{C}{r^{2}} \text { for all } v \geq C\left(\left(\left|X_{\mathbf{a}, \mathbf{b}}^{k(r)-1}\right|+(b-1)^{k(r)} r / 2\right) !\right)
$$

where $k(r)=\min \left\{k: a_{k}>2 r\right\}$.

Remark 5.9. In the above statement, $\Lambda_{i, G}$ must be interpreted as a given representative of the equivalence class $\Lambda_{i, G}$, for instance, $\Lambda_{i, G, \mathbf{u}}$ where $\mathbf{u}$ is the uniform measure on a fixed finite symmetric set of generators of the group $G$, and the constant $C$ involved in these inequalities depends on the representative. We will use this convention throughout.

Proof. The lower bound follows directly from the volume-diameter bound. See Example 3.9. Recall that $\mathfrak{m}_{k}=\left(1^{k-1}, a_{k} / 2\right)$

For the upper bound, we apply Proposition 4.11. Indeed, Lemma 5.7 shows that assumption $(\Omega)$ is satisfied with the choice

$$
J_{r}=\left\{o, \mathfrak{m}_{k(r)}\right\}, B_{r}=W(k(r), r / 4) \text { and } \boldsymbol{\Gamma}^{r}=\Gamma_{\mathbf{a}, \mathbf{b}}^{k(r)+1}
$$

where

$$
\begin{aligned}
W(k, t)= & \left\{(w, u):|w| \leq k-2, u \in\left\{1 \ldots, 2 a_{|w|+1}-1\right\}\right\} \\
& \bigcup\left(\bigcup_{|w|=k-2} \mathfrak{N}(w, t)\right) \bigcup B\left(\mathfrak{m}_{k}, t\right),
\end{aligned}
$$

and $\Gamma_{\mathbf{a}, \mathbf{b}}^{k+1}$ is the finite bubble group acting on the first $k+1$ levels of the bubble graph. Note that it follows from the various definitions that

$$
\Omega\left(J_{r}, B_{r}\right) \subset \Omega\left(\mathfrak{m}_{k(r)}, B\left(\mathfrak{m}_{k(r)}, r / 4\right)\right)
$$

Note that we abuse notation a little here by indexing $J_{r}, B_{r}, \Gamma^{r}$ by $r$ instead of an integer as in Definition 4.4. Also, in Lemma 5.7 the group $\Gamma^{r}$ is denoted by $\Gamma^{k(r)}$.

To apply Proposition 4.11 we need a $\left(J_{r}, B_{r}\right)$-admissible function $F$ charging translates of a fix set $A_{r}$ together with a test function $\psi_{r}$ on $\boldsymbol{\Gamma}^{r}$. We pick

$$
A_{r}=W(k(r), r / 8)
$$


Figure 12: The set $W(k, t)$ on a sketch of the Schreier graph $X$

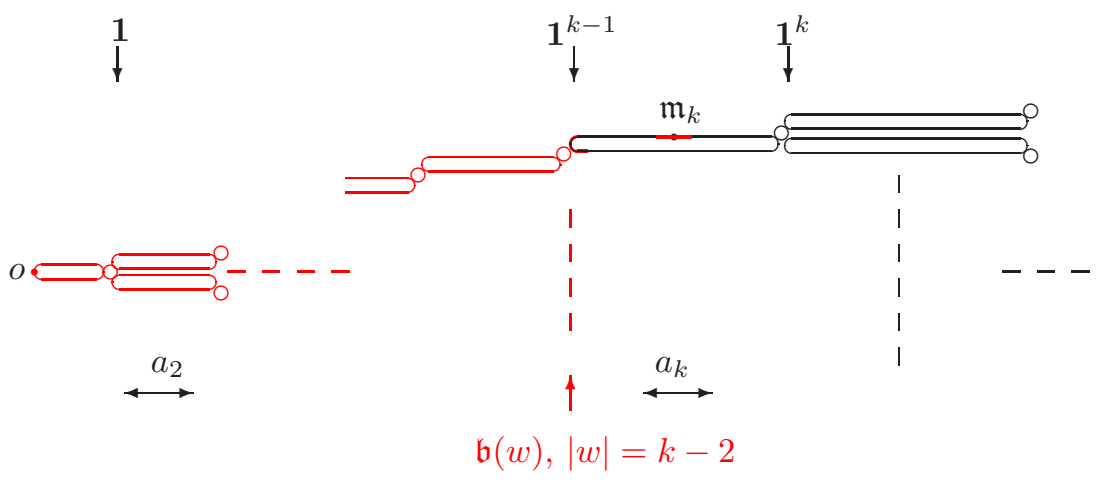

and observe that if $|t| \leq r / 8$ then

$$
\beta \alpha^{t} \cdot A_{r}=\alpha^{t} \cdot A_{r} .
$$

Set

$$
F_{r}(Y)=\left\{\begin{array}{cl}
1-8|t| / r & \text { if } Y=\alpha^{t} \cdot A_{r} \\
0 & \text { otherwise. }
\end{array}\right.
$$

This is a $\left(J_{r}, B_{r}\right)$-admissible function and, obviously,

$$
\mathcal{Q}_{\mathcal{P}_{f}(X), \mu}\left(F_{r}\right) \leq C / r^{2} .
$$

For the function $\psi_{r}$ on $\boldsymbol{\Gamma}^{r}$, we simply take $\psi_{r} \equiv 1$.

To finish the proof, we need to estimate the size of the set

$$
\bar{\Omega}\left(J_{r}, B_{r}\right) \subset \bar{\Omega}\left(\mathfrak{m}_{k(r)}, B\left(\mathfrak{m}_{k(r)}, r / 4\right)\right) .
$$

By Lemma [5.6] any element $\gamma \in \bar{\Omega}\left(\mathfrak{m}_{k(r)}, B\left(\mathfrak{m}_{k(r)}, r / 4\right)\right.$ is determined by its action on $A_{r}$ and the image of $A_{r}$ is contained in $W(k(r), r / 2)$. Consequently,

$$
\mid \bar{\Omega}\left(\mathfrak{m}_{k(r)}, B\left(\mathfrak{m}_{k(r)}, r / 4\right)|\leq| W(k(r), r / 2) \mid ! \leq\left(\left|X_{\mathbf{a}, \mathbf{b}}^{k(r)-1}\right|+(b-1)^{k(r)} r / 2\right) !\right.
$$

\subsection{Bubble group examples}

We now discuss these results for a variety of examples of sequences $\mathbf{a}=\left(a_{n}\right)_{1}^{\infty}$ when $b_{i}=3$ for all $i$. The quality of the results depends on whether $s_{k}=$ $\sum_{1}^{k} a_{i} \asymp a_{k}$ or not. The partial sum $s_{k}$ which is equal, essentially, to the distance between the root $o$ and the branching cycles $\mathfrak{b}(w)$ with $|w|=k-1$, that is, the branching cycles at level $k$. If $a_{k}$ is much smaller than $s_{k}$ then the 
upper and lower bounds for $\Lambda_{1, G}$ and $\Lambda_{2, G}$ in Theorem 5.8 do not match. See the first example below. If, $a_{k} \asymp s_{k}$ then the bound matches and we obtain good results.

Example 5.10. Assume that $a_{k}=k$. Then $s_{k}=k(k+1) / 2$. If $r \in\left[s_{k}, s_{k+1}\right]$, $\left|B_{X}(o, r)\right| \asymp(1+k) 2^{k}$. If $\log v \asymp\left|B_{X}(o, r)\right| \asymp(1+k) 2^{k}$, we obtain

$$
C(\log \log v)^{-1} \geq \Lambda_{1, G}(v) \geq(C \log \log v)^{-2}
$$

and

$$
(C \log \log v)^{-4} \leq \Lambda_{2, G}(v) \leq C(\log \log v)^{-2} .
$$

Example 5.11. Fix $\beta>0$, take $a_{k}=\left\lfloor 2^{\beta k}\right\rfloor$. Then $s_{k} \asymp a_{k} \asymp 2^{\beta k}$ and, for $r \asymp 2^{\beta k}$, we have $\left|B_{X}(o, t)\right| \asymp 2^{(\beta+1) k}$. It follows that Theorem 5.8 gives

$$
\Lambda_{1, G}^{2}(v) \asymp \Lambda_{2, G}(v) \asymp\left(\frac{\log \log v}{\log v}\right)^{2 \beta /(\beta+1)} .
$$

From this estimate on $\Lambda_{2, G}$, we deduce also that

$$
\Phi_{G}(n) \simeq \exp \left(-n^{\frac{\beta+1}{3 \beta+1}}(\log n)^{\frac{2 \beta}{3 \beta+1}}\right) .
$$

In particular, as $\beta$ varies in $(0, \infty)$, the exponent $\frac{\beta+1}{3 \beta+1}$ varies in $\left(\frac{1}{3}, 1\right)$.

Example 5.12. Assume that $a_{k}=\left\lfloor e^{f(k)}\right\rfloor$ where $f$ is positive increasing function such that $f^{-1}$ is a regularly varying function of index strictly less than 1 (including, possibly, 0). This implies that $s_{k} \asymp a_{k}$ and for $r \in\left[a_{k} / 4,4 a_{k+1}\right]$ with $k$ large enough, we have $\left|B_{X}(o, r)\right| \asymp r 2^{k} \asymp r 2^{f^{-1}(\log r)}$. Hence, if $\log v \asymp$ $r 2^{f^{-1}(\log r)} \log r$, we have

$$
\Lambda_{1, G}(v) \geq \frac{1}{C r} \geq \frac{c 2^{f^{-1}(c \log \log v)} \log \log v}{\log v} .
$$

Also, for $k$ large enough, we must have $k(r) \leq k+1$. It follows that

$$
\left[\left|X_{\mathbf{a}, \mathbf{b}}^{k(r)-1}\right|+(b-1)^{k(r)} r / 2\right] \asymp 2^{k} r
$$

and

$$
\left(\left|X_{\mathbf{a}, \mathbf{b}}^{k(r)-1}\right|+(b-1)^{k(r)} r / 2\right) ! \leq e^{C(\log r) 2^{k} r} .
$$

Hence, assuming again that $\log v \asymp r 2^{f^{-1}(\log r)} \log r$,

$$
\Lambda_{2, G}(v) \leq \frac{C}{r^{2}} \leq C^{\prime}\left(\frac{2^{f^{-1}\left(C^{\prime} \log \log v\right)} \log \log v}{\log v}\right)^{2} .
$$

In particular, if $2^{f^{-1}}$ is regularly varying, then we have

$$
\Lambda_{1, G}(v)^{2} \asymp \Lambda_{2, G}(v) \asymp\left(\frac{2^{f^{-1}(\log \log v)} \log \log v}{\log v}\right)^{2}
$$


and

$$
\Phi_{G}(n) \simeq \exp \left(-n^{1 / 3}\left(2^{f^{-1}(\log n)} \log n\right)^{2 / 3}\right) .
$$

As an explicit example, take $2^{f^{-1}(t)}=t^{\kappa}, \kappa>0$, (that is, $\left.f(t)=2^{\kappa^{-1} t}\right)$. In this case, we have

$$
\Phi_{G}(n) \simeq \exp \left(-n^{1 / 3}(\log n)^{2(1+\kappa) / 3}\right) .
$$

Finally, if $f(t)=t^{\kappa}$ with $\kappa>1$, a slightly more careful computation is needed but the end result is that, in that case,

$$
\Lambda_{1, G}(v)^{2} \asymp \Lambda_{2, G}(v) \asymp\left(\frac{2^{(\log \log v)^{1 / \kappa}} \log \log v}{\log v}\right)^{2}
$$

and

$$
\Phi_{G}(n) \simeq \exp \left(-n^{1 / 3}(\log n)^{2 / 3} 2^{\frac{2}{3}(\log n)^{1 / \kappa}}\right) .
$$

\subsection{Amenability}

In this section we prove the following statement.

Proposition 5.13. Assume that the sequence $\mathbf{a}=\left(a_{n}\right)$ satisfies $\lim \inf a_{n}=\infty$. For an arbitrary $\mathbf{b}=\left(b_{i}\right)_{1}^{\infty}, b_{i} \geq 2$, the bubble group $\Gamma_{\mathbf{a}, \mathbf{b}}$ is amenable.

Our goal is to apply the general technique of Section 4.1 and the main ingredients used for this purpose are versions of Lemmas 5.6 and 5.7 Under assumption (A), these lemmas apply to any level $k$ in the Schreier graph. The problem we face in the present setting is to find appropriate levels $k$ where the same ideas can be applied.

Given $r \geq 1$, we say that a level $k$ is appropriate for $r$ if there exists $k^{\prime}<k$ so that

1. $\inf _{j \geq k^{\prime}}\left\{a_{j-1}\right\}>r$

2. For any $b \leq r$ such that $b=b_{i}$ for some $i \geq k$, there exist $k(b) \in\left\{k^{\prime}, k^{\prime}+\right.$ $1, \ldots, k-1\}$ such that $b_{k(b)}=b$.

3. If there exists $i \geq k$ such that $b_{i}>r$, then there exists $k_{*} \in\left\{k^{\prime}, k^{\prime}+\right.$ $1, \ldots, k-1\}$ such that $b_{k_{*}}>r$.

Since $\lim \inf a_{n}=\infty$, it is easy to see that, for any $r$, there is a $r$-appropriate level $k$ (with a finite $k$ ).

At level $k_{*}$ consider the points $\mathfrak{m}_{k_{*}}=\left(1^{k_{*}-1}, a_{k_{*}} / 2\right)$ and $\mathfrak{n}_{k_{*}}=\left(1^{k_{*}-2}, a_{k_{*}-1}\right)$. Let $B\left(\mathfrak{m}_{k_{*}}, r / 4\right)$ be the ball of radius $r / 4$ around $\mathfrak{m}_{k_{*}}$ in $X$.

On the branching cycle $\mathfrak{b}\left(1^{k_{*}-2}\right)$ (i.e, the cycle which contains $\left.\mathfrak{n}_{k}\right)$, let $J_{*}(r)$ be arc of radius $r / 4$ centered at $\mathfrak{n}_{k_{*}}=\left(1^{k_{*}-2}, a_{k_{*}-1}\right)$. Each of the point in $J_{*}(r) \backslash\left\{\mathfrak{n}_{k_{*}}\right\}$ belongs to a unique bubble of total length $2 a_{k_{*}}$ whereas $\mathfrak{n}_{k_{*}}$ belongs 
to a bubble of length $2 a_{k_{*}-1}$. On each of the bubbles containing $x \in J_{*}(r)$, let $\mathcal{I}_{*}(x, r)$ the arc of radius $r / 4$ centered at $x$. Let

$$
\mathcal{U}_{k_{*}}(r)=\bigcup_{x \in J_{*}(r)} \mathcal{I}_{*}(x, r)
$$

Figure 13: Sketch showing $\mathfrak{m}_{k_{*}}, J_{*}(r)$ and $\mathcal{U}_{k_{*}}(r)$
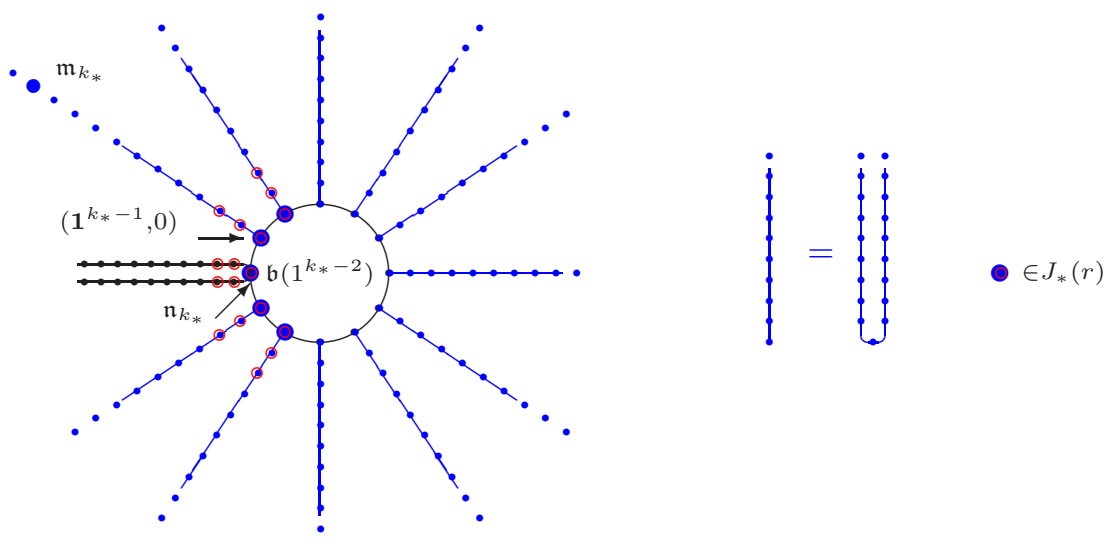

Lemma 5.14 (Local Embeddings). For any $r \geq 1$, let $k$ be an $r$-appropriate level. The restriction of the map $\vartheta_{k}=\theta_{k+1}: \mathbf{S}^{(\infty)} \rightarrow \Gamma_{\mathbf{a}, \mathbf{b}}^{k+1}$ to the set

$$
\Omega_{k}=\Omega\left(\mathfrak{n}_{k_{*}}, \mathcal{U}_{k_{*}}(r)\right) \cap \Omega\left(\mathfrak{m}_{k_{*}}, B\left(\mathfrak{m}_{k_{*}}, r / 4\right)\right.
$$

satisfies the conditions in part 2 of Assumption $(\Omega)$.

Proof. The proof is along the same lines as the proof of Lemma 5.7. The key ingredient is an identification of orbits similar to Lemma 5.6 which we explain below.

Recall that

$$
\mathfrak{N}(w, r)=\{x \in X: d(x, \mathfrak{b}(w)\} \leq r\} .
$$

For any $w$ of length $|w|=s$ with $s \geq k$ such that the branching cycle $\mathfrak{b}(w)$ is of size $b$ at most $r$, we have an obvious bijective map

$$
\iota_{k(b)}^{w}: \mathfrak{N}(w, r) \mapsto \mathfrak{N}\left(1^{k(b)-1}, r\right)
$$

which can be used to identify these vertex sets.

For any $w$ of length $|w|=s$ with $s \geq k$ such that the branching cycle $\mathfrak{b}(w)$ is of size $b$ greater than $r$ and for any $w_{s+1} \in\{0\} \cup\left\{1, \ldots, b_{s}-1\right\}$, let $\mathcal{W}_{w_{s+1}}(w, r)$ be the union of the bubble arcs of radius $r$ centered at the bubble 
roots $y=(w z, 0)$ or $\left(w, a_{s}\right)$ with $d\left(\left(w w_{s+1}, 0\right), y\right)<r / 2$ if $w_{s+1} \in\left\{1, \ldots, b_{s}-1\right\}$ and $d\left(\left(w, a_{s}\right), y\right)<r / 2$ if $w_{s+1}=0$. Define the map

$$
\iota_{*}^{w, w_{s+1}}: \mathcal{W}_{w_{s+1}}(w, r) \rightarrow \mathcal{W}_{0}\left(1^{k_{*}-1}, r\right)
$$

as follows. If $w_{s+1}=0$, identify $\mathcal{W}_{0}(w, r)$ with $\mathcal{W}_{0}\left(1^{k_{*}-1}, r\right)$ in the obvious way. If $w_{s+1} \neq 0$, use the same obvious identification after having rotated $\mathcal{W}_{w_{s+1}}(w, r)$ along the branching cycle $\mathfrak{b}(w)$ to bring the point $\left(w w_{s+1}, 0\right)$ to $\left(w, a_{s}\right)$.

For any $t \geq k$ and any vertex $x=(w, u)$ with $|w|=t, w=w_{1} \ldots w_{t}$ and $0 \leq u \leq 2 a_{t}-1$ (i.e., any vertex at level $t$ ), let $w^{\prime}=w_{1} \ldots w_{t-1}$ be the parent of $w$. For any such $x$ we define a "reference" point $\hat{x}$ and a map $\hat{\iota}_{x}$ that carries bijectively a certain neighborhood of $x$ to a similar neighborhood of $\hat{x}$. The following specifies case by case how to construct $\hat{x}$ and $\hat{\iota}_{x}$.

- If $d\left(x,\left\{(w, 0),\left(w, a_{t}\right)\right)\right)>r / 4$ then $\hat{x}=\mathfrak{m}_{k_{*}}$. The map $\hat{\iota}_{x}$ takes the arc of radius $r / 4$ centered $x$ to the similar arc centered at $\hat{x}=\mathfrak{m}_{k_{*}}$.

- If $d(x,(w, 0)) \leq r / 4$ and $\mathfrak{b}\left(w^{\prime}\right)$ is such that its size is greater than $r$ then $\hat{x}=\left(1^{k_{*}-1}, a_{k_{*}}+u\right)$ if $0 \leq u \leq r / 4$ and $\hat{x}=\left(1^{k_{*}-1}, a_{k_{*}}-2 a_{t}+u\right)$ if $u>a_{t}$. In this case, set $\hat{\iota}_{x}=\iota_{*}^{w^{\prime}, w_{t}}$.

- If $d(x,(w, 0)) \leq r / 4$ and $\mathfrak{b}\left(w^{\prime}\right)$ is such that its size $b$ is at most $r$ then $\hat{x}=\left(1^{k(b)-1} w_{t}, u\right)$ if $0 \leq u \leq r / 4$ and $\hat{x}=\left(1^{k(b)-1} w_{t}, a_{k(b)}-2 a_{t}+u\right)$ if $u>a_{t}$. In this case, set $\hat{\iota}_{x}=\iota_{k(b)}^{w^{\prime}}$.

- If $d\left(x,\left(w, a_{t}\right)\right) \leq r / 4$ and $\mathfrak{b}(w)$ is such that its size is greater than $r$ then $\hat{x}=\left(1^{k_{*}-1}, a_{k_{*}-1}+u-a_{t}\right)$. In this case, set $\hat{\iota}_{x}=\iota_{*}^{w, 0}$.

- If $d\left(x,\left(w, a_{t}\right)\right) \leq r / 4$ and $\mathfrak{b}(w)$ is such that its size $b$ is at most $r$ then $\hat{x}=\left(1^{k(b)-1}, a_{k(b)-1}+u-a_{t}\right)$. In this case, set $\hat{\iota}_{x}=\iota_{k(b)}^{w}$.

Let $\omega=\gamma_{1} \ldots \gamma_{p}$ be a word that belongs to $\Omega_{k}$. Then, for any $1 \leq j \leq p$,

$$
\hat{\iota}_{x}\left(\gamma_{j}^{-1} \ldots \gamma_{1}^{-1} \cdot x\right)=\gamma_{j}^{-1} \ldots \gamma_{1}^{-1} \cdot \hat{x} .
$$

This is proved by inspection as in the proof of Lemma 5.6.

Proof of Proposition 5.13. Using Lemma 5.14 and Section 4.3, we build test functions that serve as witnesses for the amenability of $\mathbb{Z} \imath_{X} \Gamma$.

For $r \geq 1$, let $k$ be an $r$-appropriate level with associated $k^{\prime}, k_{*}, k(b)$ as above. Let

$$
J_{r}=\left\{o, \mathfrak{n}_{k_{*}}, \mathfrak{m}_{k_{*}+1}\right\}
$$

and

$$
\begin{aligned}
\Xi(k, t)= & \left\{(w, u):|w| \leq k^{\prime}-1, u \in\left\{1 \ldots, 2 a_{|w|+1}-1\right\}\right\} \\
& \bigcup\left(\bigcup_{|w|=k^{\prime}-1} \mathfrak{N}(w, t / 4)\right) \\
& \bigcup B\left(\mathfrak{m}_{k_{*}}, t / 4\right) \bigcup \mathcal{U}_{k_{*}}(t) .
\end{aligned}
$$


For $t \leq r$, this set is made of 3 disjoint parts $B\left(\mathfrak{m}_{k_{*}}, t / 4\right), \mathcal{U}_{k_{*}}(t)$ and the rest, and each of these parts contains exactly one of the points in $J_{k}$. Using this notation, we set

$$
B_{r}=\Xi(k, r)
$$

\section{Neumann-Segal type groups}

In [29], D. Segal constructed finitely generated branch groups that contain every non-abelian finite simple group as homomorphic image, and proved that there is no gap in subgroup growth of finitely generated groups. A similar construction also appeared in P. Neumann 23. A version of these constructions can be described as follows.

Given a sequence of finite sets $\left(X_{j}\right)_{1}^{\infty}$, we obtain a rooted tree $\mathcal{T}=\mathcal{T}_{0}$ with root $\emptyset$, first level $X_{1}$, and so that each vertex at level $i$ has children encoded by a copy of $X_{i+1}$.

Let $\left(G_{i}, X_{i}\right)_{i=1}^{\infty}$ be a sequence of groups acting transitively on finite sets $X_{i}$ with the property that each $G_{i}$ is $k$-generated and marked with a generating $k$-tuple $\left(s_{i, 1}, s_{i, 2}, \ldots, s_{i, k}\right)$. In each $X_{i}$, choose two distinct points $x_{i}, y_{i}$. Define automorphisms $\alpha_{i, j}$ and $\beta_{i, j}$ of the tree $\mathcal{T}_{i}=\left(X_{i+1}, X_{i+2}, \ldots\right)$ recursively as follows. For $i \geq 0$, the automorphism $\alpha_{i, j}$ is a rooted permutation

$$
\alpha_{i, j}(x w)=s_{i+1, j}(x) w,
$$

and $\beta_{i, j}$ is a directed automorphism defined at $x w$ with $x \in X_{i+1}, w \in\left(X_{i+2}, X_{i+3}, \ldots\right)$

$$
\beta_{i, j}(x w)=\left\{\begin{array}{cc}
x_{i+1} \beta_{i+1, j}(w) & \text { if } x=x_{i+1} \\
y_{i+1} \alpha_{i+1, j}(w) & \text { if } x=y_{i+1} \\
x w & \text { otherwise }
\end{array}\right.
$$

For each $i \geq 0$, let

$$
\Gamma_{i}=\left\langle\alpha_{i, j}, \beta_{i, j}, 1 \leq j \leq k\right\rangle
$$

be the group generated by $\alpha_{i, j}, \beta_{i, j}, 1 \leq j \leq k$, acting on the subtree $\mathcal{T}_{i}=$ $\left(X_{i+1}, X_{i+2}, \ldots\right)$.

Set $\alpha_{j}=\alpha_{0, j}, \beta_{j}=\beta_{0, j}, 1 \leq j \leq k$ and let

$$
\Gamma=\Gamma_{0}=\left\langle\alpha_{j}, \beta_{j}, 1 \leq j \leq k\right\rangle
$$

be the group generated by the rooted automorphisms $\alpha_{j}$ and directed automorphisms $\beta_{j}, 1 \leq j \leq k$. Such groups are called groups of Neumann-Segal type. They also belong to the class of directed tree automorphism groups. Specifically, the generators $\beta_{j}$ are directed along the ray $o=x_{1} x_{2} \ldots$ in $\mathcal{T}$. In particular, $\beta_{j}$ leave this ray invariant. See [6, 16. They are also branch groups. See [15, 16]. 
Figure 14: The level 3 Schreier graph of the Neumann-Segal group $\Gamma$ with sequence $\left(l_{n}\right)$ starting with $(2,4,4)$.

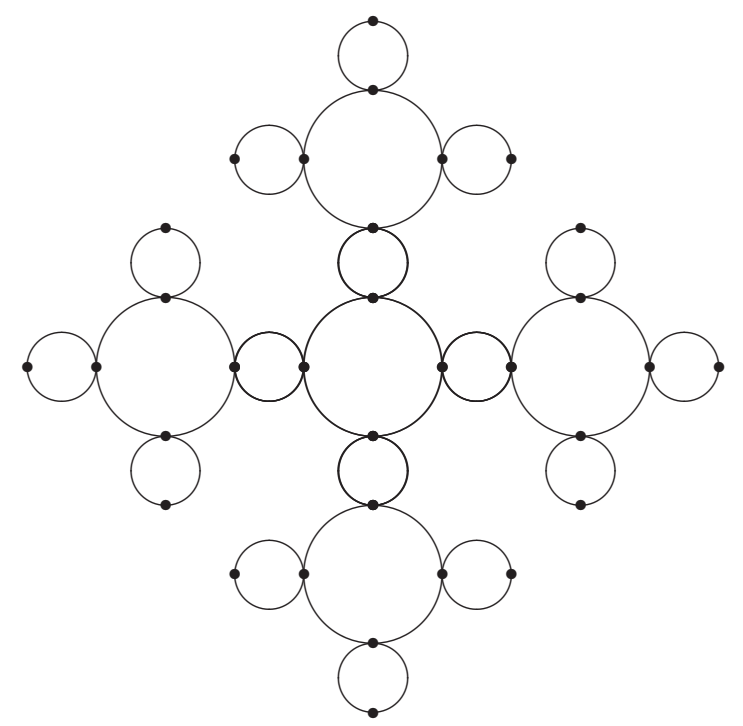

\subsection{Neumann-Segal groups with cyclic $G_{i}$}

In what follows we focus on the (very) special case where each $G_{i}$ is a finite cyclic group of even order $l_{i}$ marked with one generator $s_{i}$, and $X_{i}=G_{i}=$ $\left\{1, \ldots, 2 l_{i}-1\right\}$, that is, $G_{i}$ acts on itself by multiplication. We also pick

$$
x_{i}=0 \text { and } y_{i}=l_{i} / 2 .
$$

These are also examples of generalized Fabrykowski-Gupta groups. See [13.

As in the case of other directed groups, the Schreier graph $\mathcal{S}$ of $o=00 \cdots=$ $0^{\infty}$ can be constructed via a global substitution procedure which we now describes. See [16] and the references therein.

For $i=1$, let $\mathcal{S}_{1}$ be the cycle $\left\{0, \ldots, 2 l_{1}-1\right\}$. For $i \geq 2$, the Schreier graph $\mathcal{S}_{i}$ is constructed by taking the cycle $G_{i}$ and $l_{i}$ copies of the Schreier graph $\mathcal{S}_{i-1}$ constructed in the previous step. For each $z \in G_{i}$, attach a copy of the graph $\mathcal{S}_{i-1}$ to $z$ by identifying $z$ with the vertex labeled $0 \ldots 0 y_{i-1}$ (with $i-1$ zeros) in that copy of $\mathcal{S}_{i-1}$. Finally, relabel the vertex originally labeled $\omega$ in this copy of $\mathcal{S}_{i-1}$ by giving it the label $\omega z$ in $\mathcal{S}_{i}$.

The diameters of the graphs $\mathcal{S}_{i}, i \geq 1$, satisfy

$$
\operatorname{Diam}\left(\mathcal{S}_{i}\right)=2 \operatorname{Diam}\left(\mathcal{S}_{i-1}\right)+l_{i} / 2,
$$

therefore

$$
\operatorname{Diam}\left(\mathcal{S}_{i}\right)=\sum_{m=1}^{i} 2^{i-m-1} l_{m}
$$


The cardinality of $\mathcal{S}_{i}$ is

$$
V_{i}=\left|\mathcal{S}_{i}\right|=\prod_{1 \leq m \leq i} l_{m}
$$

The infinite Schreier graph $\mathcal{S}$ is the orbital Schreier graph of the ray $0^{\infty}$. Viewed from $0^{\infty}$, the finite Schreier graphs $\mathcal{S}_{i}$ describe growing pieces of $\mathcal{S}$.

In 14, E. Fink studies some algebraic properties of a similar class of group where the choice of the point $y_{i}$ is different, namely, instead of $y_{i}=l_{i} / 2$, Fink makes the choice $y_{i}=1$, a neighbor of $x_{i}=0$. She also consider mostly the case when $\left(l_{i}\right)$ is a sequence of pairwise distinct primes. One of Fink's results is that every proper quotient of the groups she considers are solvable. This and many of the other algebraic properties she proves carry over without difficulties to the groups we consider here. See also [15, 29].

The volume growth properties of Neumann-Segal groups is a subject of great interest. The cases that has been most studied is when the level- $i$ permutation group $G_{i}$ is the full alternating group on $X_{i}$. See [33, 32, 7] and the references therein. If the sequence $\left|X_{i}\right|$ is bounded and $\left|X_{i}\right| \geq 5$ then the volume growth is exponential ([7]). If $\left|X_{i}\right|$ is unbounded, the group contains a free group on 2 generators $([32])$.

The most striking result is perhaps the fact that when $\left|X_{i}\right|=3$ for all $i$, the group has subsexponential volume growth (in this case, $G_{i}=A_{3}$ is the same as $G_{i}$ is cyclic!). This was first announced in [13. Explicit bounds are given in 3 .

In general, we understand little about the volume growth of the groups $\Gamma$ we consider here. We note that [14, Proposition 5.4] is in error and so is the proof of [14, Theorem 5.5] which relies on it. The proof of the following lemma is along the same lines as to the proof of the volume lower bound in [3].

Lemma 6.1. For any even sequence $\left(l_{i}\right)_{1}^{\infty}$, we have

$$
\left|B_{\Gamma}\left(V_{i} r\right)\right| \geq 2^{2^{-i-4} V_{i} r}, \quad 4 \leq r \leq\left(l_{i+1} / 2\right)-1, \quad V_{i}=l_{1} \ldots l_{i}, \quad i \geq 1
$$

In particular, if $\liminf _{i \rightarrow \infty} l_{i} \geq 2^{\kappa}$, then

$$
\left|B_{\Gamma}(r)\right| \gtrsim \exp \left(r^{(\kappa-1) / \kappa}\right) .
$$

Proof. First we show that, for any $r \geq 1$,

$$
\left(\left|B_{\Gamma_{i+1}}(r)\right|^{l_{i} / 2} \leq \mid B_{\Gamma_{i}}\left((1+1 /(2 r)) r l_{i}\right) .\right.
$$

This will follow if we can show that there are elements $\left(g_{x}\right)_{0 \leq x \leq l_{i}-1} \sigma$ in $B_{\Gamma_{i}}\left(r l_{i}\right)$ with $\left(g_{x}\right)_{0 \leq x \leq\left(l_{i} / 2\right)-1}$ arbitrarily chosen in $B_{\Gamma_{i+1}}(r)^{l_{i} / 2}$. Recall that $\Gamma_{i} \subset \Gamma_{i+1} \chi_{X_{i}}$ $G_{i}$ where $G_{i}=\left\{0, \ldots, l_{i}-1\right\}$ is the cyclic group of order $l_{i}$ with $\alpha_{i}=(1, \ldots, 1) \sigma_{i}$ where $\sigma_{i}$ the cyclic permutation and $\beta_{i}=\left(\beta_{i+1}, 1 \ldots, 1, \alpha_{i+1}, 1, \ldots, 1\right)$ id with $\alpha_{i=1}$ in position $l_{i} / 2$. The key point is that $l_{i}$ is even with $\beta_{i_{+}}$and $\alpha_{i+1}$ at opposite locations. By inspection, it is easy to produce the desired elements while using $\alpha_{i}$ to move along the cycle clockwise. For instance, consider the case when

$$
g_{0}=\alpha_{i+1}^{n_{0}^{0}} \beta_{i+1}^{n_{1}^{0}}, \quad g_{\left(l_{i} / 2\right)-1}=\beta_{i+1}^{n_{1}^{\left(l_{i} / 2\right)-1}}
$$


and $g_{x}=1$ if $0<x<\left(l_{i} / 2\right)-1$. It takes

$$
\left(l_{i} / 2\right)+\left|n_{0}^{0}\right|+\left(l_{i} / 2\right)+\left|n_{1}^{0}\right|+\left(\left(l_{i} / 2\right)-1\right)+\left|n_{1}^{\left(l_{i} / 2\right)-1}\right|
$$

where each $\left(l_{i} / 2\right)$ or $\left(l_{1} / 2\right)-1$ represent a run of cycling move using $\alpha_{i}$ and the $\left|n_{j}^{x}\right|$ counts uses of $\beta_{i}$ to insert either $\alpha_{i+1}$ (when $j$ is even) or $\beta_{i+1}$ (when $j$ is odd). The total number of moves for this example is $\left(l_{i} / 2\right)+2\left(\left(l_{i} / 2\right)-1 \sum_{x}\left|g_{x}\right|\right.$. In general, an arbitrary $\left(g_{x}\right)_{0 \leq x \leq l_{i} / 2}$ with $\left|g_{x}\right| \leq r$ and a maximal number of switches from $\alpha_{i+1}$ to $\beta_{i+1}$ in any $g_{x}, 0 \leq x \leq(l-i / 2)-1$ equal to $m$ can be produce in at most

$$
\left(l_{1} / 2\right)+\left(l_{i} / 2\right) m+\sum_{0}^{l_{i} / 2-1}\left|g_{x}\right|-1
$$

This is less than

$$
\left(l_{i} / 2\right)+l_{i} r=(1+1 /(2 r)) r l_{i}
$$

as desired. By induction, this gives

$$
\left|B_{\Gamma}\left(R_{i}\right)\right| \geq\left|B_{\Gamma_{i+1}}(r)\right|^{2^{-i-1} v_{i}}
$$

where

$$
R_{i}=\left(\prod_{1}^{i}\left(1+1 /\left(2 r w_{j, i}\right)\right) l_{j}\right) r, \quad w_{j, i}=l_{j+1} \ldots l_{i}, \quad w_{i, i}=1
$$

Note that, since $l_{i} \geq 2$ and $r \geq 1$, we have $\prod_{1}^{i}\left(1+1 /\left(2 r w_{j, i}\right)\right) \leq e$. Hence $R_{i} \leq e V_{i} r$ and we have

$$
\left|B_{\Gamma}\left(e V_{i} r\right)\right| \geq\left|B_{\Gamma_{i+1}}(r)\right|^{2^{-i-1} V_{i}} .
$$

Further, a simple version of the previous argument shows that

$$
\left|B_{\Gamma_{i+1}}(r)\right| \geq 2^{r / 2}, \quad 1 \leq r \leq\left(l_{i+1} / 2\right)-1 .
$$

Hence, we obtain

$$
\left|B_{\Gamma}\left(V_{i} r\right)\right| \geq 2^{2^{-i-4} V_{i} r}, \quad 4 \leq r \leq\left(l_{i+1} / 2\right)-1, \quad i=1,2, \ldots \ldots
$$

\subsection{Lower bound on the isoperimetric profile}

By applying Proposition 3.6 in the case of the above group $\Gamma$, we obtain a lower bound on the isoperimetric of $\mathbb{Z} \imath_{\mathcal{S}} \Gamma$.

Corollary 6.2 (Corollary of Proposition 3.6). For any sequence of even integers $l_{i}$ with $\lim \sup l_{i}=\infty$, We have

$$
\Lambda_{1, \mathbb{Z} \imath_{\mathcal{S}} \Gamma}(v) \geq \frac{1}{C r} \text { for all } v \leq C^{-1} r^{\left|B_{\mathcal{S}}\left(0^{\infty}, r\right)\right| / C} .
$$


Example 6.3. Let $l_{n}=2 n^{d}, n \geq 1$, for some integer $d$. Diameter and volume are given by $\operatorname{Diam}\left(\mathcal{S}_{n}\right) \asymp 2^{n}$ and $\left|\mathcal{S}_{n}\right|=V_{n}=2^{n}(n !)^{d}$. This gives

$$
\Lambda_{1, \mathbb{Z}_{\mathcal{S}} \Gamma}(v) \gtrsim c 2^{-\frac{1}{d} \frac{\log \log v}{\log \log \log v}\left(1+2 \frac{\log \log \log \log v}{\log \log \log v}\right)}
$$

If instead we assume that $l_{n}=2^{1+\left\lfloor n^{\gamma}\right\rfloor}$ with $\gamma>0$ then $\operatorname{Diam}\left(\mathcal{S}_{n}\right) \asymp 2^{n}$ if $\gamma \in(0,1), \operatorname{Diam}\left(\mathcal{S}_{n}\right) \asymp n 2^{n}$ if $\gamma=1$ and $\operatorname{Diam}\left(\mathcal{S}_{n}\right) \asymp 2^{n^{\gamma}}$ if $\gamma>1$. Also, $\log _{2}\left(V_{n}\right) \sim(1+\gamma)^{-1} n^{1+\gamma}$. The bound of the previous corollary yields

$$
\Lambda_{1, \mathbb{Z} Z \Gamma}(v) \geq \frac{1}{C 2^{n^{\max \{1, \gamma\}}}} \text { for all } v \leq C^{-1} \exp \left(2^{[(1+\epsilon)(1+\gamma)]^{-1} n^{\gamma+1}}\right),
$$

for any $\epsilon>0$. That is,

$$
\Lambda_{1, \mathbb{Z} L \Gamma}(v) \geq c 2^{-(1+\epsilon)\left[(1+\gamma) \log _{2} \log v\right]^{\max \{1, \gamma\} /(1+\gamma)} .}
$$

\subsubsection{First lower bound for $\Gamma$ itself}

This subsection shows how the very general Proposition 3.11 can be applied to the Neumann-Segal groups $\Gamma=\langle\alpha, \beta>$, under certain hypotheses on the length sequence $\left(l_{n}\right)_{1}^{\infty}$.

In order to apply Proposition 3.11 for each level $n$, we need to find an element $\rho_{n}$ in $\Gamma$ that belongs to the rigid stabilizer $\operatorname{rist}_{\Gamma}\left(u_{n}\right)$ of a vertex $u_{n}$ at level $n$ and to control the length $\left|\rho_{n}\right|_{\Gamma}$ of $\rho_{n}$ in $\Gamma$. For this purpose, we assume that $\overline{\lim }_{n \rightarrow \infty} l_{n}=\infty$. Let

$$
u_{n}=0^{n} \in \mathcal{S}_{n}
$$

and

$$
\rho_{n}=\beta^{M_{n+1}},
$$

where $M_{n+1}$ is a chosen common multiple of $\left\{l_{2}, \ldots, l_{n+1}\right\}$. By direct inspection, we have

$$
\rho_{n}=\left(\beta_{n+1}^{M_{n+1}}, 1, \ldots, 1\right) \mathrm{id}
$$

and $\beta_{n+1}^{M_{n+1}}$ is non-trivial because $\beta$ is of infinite order thanks to the assumption that $\left(l_{i}\right)$ is unbounded.

Corollary 6.4 (Corollary of Proposition 3.11). Suppose $\overline{\lim }_{n \rightarrow \infty} l_{n}=\infty$, let $M_{n+1}$ denote a chosen common multiple of $\left\{l_{2}, \ldots, l_{n+1}\right\}$. Then there exists a constant $C \geq 1$ such that

$$
\Lambda_{1, \Gamma}(v) \geq \frac{1}{C \max \left\{M_{n+1}, r\right\}} \text { for all } v \leq C^{-1} 2^{\left|B_{\mathcal{S}_{n}}\left(u_{n}, r\right)\right| / C} .
$$

Remark 6.5. We can always take

$$
M_{n+1}=V_{n+1}=l_{1} \ldots l_{n+1} \text { and } r=\operatorname{Diam}\left(\mathcal{S}_{n}\left(u_{n}\right)\right) \leq V_{n+1} .
$$

This gives

$$
\Lambda_{1, \Gamma}(v) \geq \frac{1}{C V_{n+1}} \text { for all } v \leq C^{-1} 2^{V_{n} / C}
$$

but this estimate is too weak to be useful because $V_{n+1} / V_{n}=l_{n+1}$ is unbounded. 
Example 6.6. Assume that $l_{n}=2^{1+\left\lfloor n^{\gamma}\right\rfloor}, n \geq 1$, with $\gamma>0$. This gives $\operatorname{Diam}\left(\mathcal{S}_{n}\right) \asymp 2^{n}$ if $\gamma \in(0,1), \operatorname{Diam}\left(\mathcal{S}_{n}\right) \asymp n 2^{n}$ if $\gamma=1$ and $\operatorname{Diam}\left(\mathcal{S}_{n}\right) \asymp 2^{n^{\gamma}}$ if $\gamma>1$. Also, $\log _{2}\left(V_{n}\right) \asymp(1+\gamma)^{-1} n^{1+\gamma}$ and $M_{n+1}=l_{n+1}=2^{1+\left\lfloor(n+1)^{\gamma}\right\rfloor}$. The bound of the previous corollary yields (with a different constant $C$ depending of $\gamma>0)$

$$
\Lambda_{1, \Gamma}(v) \geq \frac{1}{C 2^{(n+1)^{\max \{1, \gamma\}}}} \text { for all } v \leq C^{-1} \exp \left(2^{(1-\epsilon)(1+\gamma)^{-1} n^{\gamma+1}}\right) .
$$

That is,

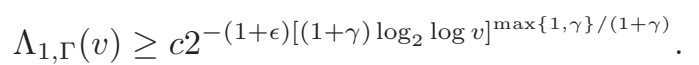

Note that this is the same bound we obtained for $\Lambda_{1, \mathbb{Z} 2 \Gamma}$ at the end of Example 6.3. Because of the appearance of the quantity $M_{n+1}$ in Corollary 6.4 when $l_{n}=2 n^{d}$ as in the first part of Example 6.3. we cannot give a lower bound similar to that obtained for $\Lambda_{1, \mathbb{Z} 2 \Gamma}$.

\subsubsection{Improved lower bound for $\Gamma$ itself}

The main drawback of Corollary 6.4 is the fact that the bound involves the quantities $M_{n}$ and $\left|B_{\mathcal{S}_{n}}\left(u_{n}, r\right)\right|$ instead of $M_{n-1}$ and $\left|B_{\mathcal{S}_{n}}\left(u_{n}, r\right)\right|$. See Remark 6.5. In this section we show that in the special case of the group $\Gamma$ studied in this section, a slightly sharper version of Proposition 3.11 can be obtained and that fixes this drawback.

Proposition 6.7 (Improved version of Corollary 6.4). Suppose $\overline{\lim }_{n \rightarrow \infty} l_{n}=\infty$, let $M_{n}$ denote a chosen common multiple of $\left\{l_{2}, \ldots, l_{n}\right\}$. Then there exists a constant $C \geq 1$ such that

$$
\Lambda_{1, \Gamma}(v) \geq \frac{1}{C \max \left\{M_{n}, r\right\}} \text { for all } v \leq C^{-1} 2^{\left|W_{n}(r)\right| / C} .
$$

Proof. In $\mathcal{S}_{n}=\mathcal{S}_{n}\left(u_{n}\right), u_{n}=0^{n}$, consider the set $W_{n}$ of those vertices $v=$ $z_{1} z_{n-1} z_{n}$ with $0 \leq z_{n} \leq\left\lfloor l_{n} / 4\right\rfloor$. Set

$$
W_{n}(r)=W_{n} \cap B_{\mathcal{S}_{n}}\left(u_{n}, r\right) .
$$

Note that $B_{\mathcal{S}_{n}}\left(u_{n}, r\right) \subset W_{n}$ if and only if $r \leq \operatorname{Diam}\left(\mathcal{S}_{n-1}\right)+l_{n} / 4$.

For each such $v$, pick $g^{v}$ such that $g^{v} u_{n}=v$ and $\left|g^{v}\right|=d_{\mathcal{S}_{n}}\left(u_{n}, v\right)$. Set $\varrho_{n}=\beta^{M_{n}}$. By construction

$$
\varrho_{n}=\left(\tilde{\varrho}_{x}\right)_{x \in \mathcal{T}^{n}} \mathrm{id}
$$

where $\tilde{\varrho}_{x}$ is the identity except at $x=u_{n}$ and $x=\bar{u}_{n}=0^{n-1}\left(l_{n} / 2\right)$. Further,

$$
\tilde{\varrho}_{u_{n}}=\beta_{n+1}^{M_{n}} \text { and } \tilde{\varrho}_{\bar{u}_{n-1}}=\alpha_{n+1}^{M_{n}} \text {. }
$$

For $v \in W_{n}$, write $g^{v}=\left(g_{x}^{v}\right)_{x \in \mathcal{T}^{n}} \sigma^{v}$. We have $\sigma^{v}\left(u_{n}\right)=v$. Because a minimal length representation of $g^{v}$ as a word in $\alpha, \beta$ provides a geodesic from $u_{n}$ to $v$ in $\mathcal{S}_{n}$, we also have $\sigma^{v}\left(\bar{u}_{n}\right)=z_{1} \ldots z_{n-1} \bar{z}_{n}$ where $\bar{z}_{n}=z_{n}+l_{n} / 2$. Set $\bar{W}_{n}=$ 
$\left\{\sigma^{v}\left(\bar{u}_{n}\right): v \in W_{n}\right\}$ and observe that $W_{n}$ and $\bar{W}_{n}$ are disjoint subsets of $\mathcal{S}_{n}$. Then

$$
g^{v} \varrho_{n}\left(g^{v}\right)^{-1}=\left(\theta_{x}^{v}\right)_{x \in \mathcal{T}^{n} \text { id }}
$$

where all $\theta_{x}^{v}$ are trivial except two, namely,

$$
\theta_{v}^{v}=\theta_{v}^{v}=g_{v}^{v} \beta_{n+1}^{M_{n}}\left(g_{v}^{v}\right)^{-1} \text { and } \theta_{\bar{v}}^{v}=g_{\bar{v}}^{v} \alpha_{n+1}^{M_{n}}\left(g_{\bar{v}}^{v}\right)^{-1}
$$

where $\bar{v}=\sigma^{v}\left(\bar{u}_{n}\right)$.

Now, let $\zeta$ be the symmetric probability measure on the subgroup

$$
\left\langle g^{v} \varrho_{n}\left(g^{v}\right)^{-1}: v \in W_{n}\right\rangle
$$

defined be

$$
\zeta(\gamma)=\frac{1}{2\left|W_{n}(r)\right|} \sum_{v \in W_{n}(r)} \mathbf{1}_{\left\{g^{v} \varrho_{n}^{ \pm 1}\left(g^{v}\right)^{-1}\right\}}(\gamma) .
$$

As $\zeta$ has the form (3.1) on the product of cyclic groups

$$
X=\prod_{v \in W_{n}(r)}<g^{v} \varrho_{n}\left(g^{v}\right)^{-1}>,
$$

Comparison of $\zeta$ with simple random walk on $\Gamma$ and Proposition 3.1 gives the desired result.

Corollary 6.8. Suppose $\overline{\lim }_{n \rightarrow \infty} l_{n}=\infty$. Then, for $c \in(0,1)$ small enough, we have

$$
\Lambda_{1, \Gamma}(v) \geq \frac{c}{\log v} \text { for } v \in\left[2^{c^{2} V_{n}}, 2^{c V_{n}}\right] .
$$

Note that a better lower bound would be available if we knew that $\Gamma$ has exponential volume growth.

\subsection{Upper bound on the isoperimetric profile}

In this section, we provide upper bounds on $\Lambda_{2, \mathbb{Z} 2 \Gamma}$ by applying the general method explained in Section 4 .

Proposition 6.9. There exists a constant $C$ such that, for any sequence $\left(l_{i}\right)_{1}^{\infty}$ of even natural numbers and any $n$, we have

$$
\Lambda_{2, \mathbb{Z} \imath_{\mathcal{S}} \Gamma}(v) \leq \frac{1}{C R_{n}} \text { for any } v \geq C R_{n}^{C V_{n}}
$$

where $R_{n}=\sum_{1}^{n} 2^{n-j-2} l_{j}$ and $V_{n}=l_{1} \ldots l_{n}$.

The quantity $R_{n}$ which appears here is (essentially) the resistance between the root $o=0^{\infty}$ and the set $\mathcal{S}_{n}^{c}$ in the Schreier graph $\mathcal{S}$. 
Example 6.10. Let $l_{n}=2 n^{d}, n \geq 1$ for some integer $d$. Resistance and volume are given by $R_{n} \asymp 2^{n}$ and $\left|\mathcal{S}_{n}\right|=V_{n}=2^{n}(n !)^{d}$. This gives

$$
\Lambda_{2, \mathbb{Z} \imath_{S} \Gamma}(v) \lesssim C 2^{-\frac{1}{d} \frac{\log \log v}{\log \log \log v}\left(1+\frac{\log \log \log \log v}{2 \log \log \log v}\right)} .
$$

If instead we assume that $l_{n}=2^{1+\left\lfloor n^{\gamma}\right\rfloor}$ with $\gamma>0$ then $R_{n} \asymp 2^{n}$ if $\gamma \in(0,1)$, $R_{n} \asymp n 2^{n}$ if $\gamma=1$ and $R_{n} \asymp 2^{n^{\gamma}}$ if $\gamma>1$. Also, $\log _{2}\left(V_{n}\right) \asymp(1+\gamma)^{-1} n^{1+\gamma}$. This gives

$$
\Lambda_{2, \mathbb{Z} L \Gamma}(v) \leq \frac{C}{2^{n^{\max \{1, \gamma\}}}} \text { for all } v \geq C \exp \left(2^{[(1-\epsilon)(1+\gamma)]^{-1} n^{\gamma+1}}\right) .
$$

That is,

$$
\Lambda_{2, \mathbb{Z} 2 \Gamma}(v) \leq 2^{-(1-\epsilon)\left[(1+\gamma) \log _{2} \log v\right]^{\max \{1, \gamma\} /(1+\gamma)}} .
$$

Proposition 6.11. There exists a constant $C$ such that, for any sequence $\left(l_{i}\right)_{1}^{\infty}$ of even natural numbers and any $n$, we have

$$
\Lambda_{2, \mathbb{Z} \iota_{\mathcal{S}} \Gamma}(v) \leq \frac{C}{r^{2}} \text { for any } v \geq \exp \left(C V_{n-1} r \log r\right)
$$

where $r \in\left(0, l_{n} / 4\right)$ and $V_{n-1}=l_{1} \ldots l_{n-1}$.

Remark 6.12. Proposition 6.11 gives a better result than Proposition 6.9 when $l_{n} \gg \sqrt{R_{n}}$ (by inspection, we always have $R_{n} \geq l_{n} / 4$ ).

Example 6.13. Consider the case when $l_{n}=2^{1+\left\lfloor n^{\gamma}\right\rfloor}$ with $\gamma>0$. If $\gamma \in(0,1)$, Proposition 6.9 gives a better than Proposition 6.11 whereas, for $\gamma \geq 1$, Proposition 6.11 yields a much better result. Namely, using $r=l_{n} / 4$, Proposition 6.11 gives

$$
\Lambda_{2, \mathbb{Z} \backslash \Gamma}(v) \leq \frac{C}{2^{2 n^{\max \{1, \gamma\}}}} \text { for all } v \geq C \exp \left(2^{[(1-\epsilon)(1+\gamma)]^{-1} n^{\gamma+1}}\right) .
$$

That is,

$$
\Lambda_{2, \mathbb{Z} \Gamma \Gamma}(v) \leq 2^{-2(1-\epsilon)\left[(1+\gamma) \log _{2} \log v\right]^{\max \{1, \gamma\} /(1+\gamma)} .}
$$

The next theorem applies to $\Gamma$ as well as to $\mathbb{Z} \imath_{\mathcal{S}} \Gamma$.

Theorem 6.14. For $l_{n}=2^{1+\left\lfloor n^{\gamma}\right\rfloor}, n \geq 1, \gamma \geq 1$, we have, for any $\epsilon>0$ and constants $c, C$ that depends only on $\epsilon$,

$$
\begin{gathered}
c 2^{-(1+\epsilon)\left[(1+\gamma) \log _{2} \log v\right]^{\gamma /(1+\gamma)}} \leq \Lambda_{1, \Gamma}(v) \leq C 2^{-(1-\epsilon)\left[(1+\gamma) \log _{2} \log v\right]^{\gamma /(1+\gamma)},} \\
c 2^{-2(1+\epsilon)\left[(1+\gamma) \log _{2} \log v\right]^{\gamma /(1+\gamma)}} \leq \Lambda_{2, \Gamma}(v) \leq C 2^{-2(1-\epsilon)[(1+\gamma) \log 2 \log v]^{\gamma /(1+\gamma)}},
\end{gathered}
$$

and

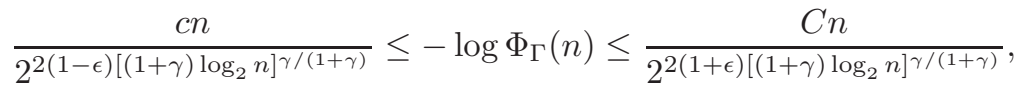

as well as the same estimates for $\Lambda_{1, \mathbb{Z} \imath_{\mathcal{S}} \Gamma}, \Lambda_{2, \mathbb{Z}_{\mathcal{S}} \Gamma}$ and $\Phi_{\mathbb{Z} \mathfrak{Z}_{\mathcal{S}} \Gamma}$. 
Proof of Proposition 6.9. First, we explain how we arrange for assumption $(\Omega)$ to be satisfied. For each $n$, consider the copy of $\mathcal{S}_{n}$ in $\mathcal{S}$ which is anchored at $0^{\infty}$. In $\mathcal{S}$, let $B_{n}$ (resp. $\bar{B}_{n}$ ) be the set of those points that are (strictly) closer to $0^{\infty}$ than to $0^{n-1}\left(l_{n} / 2\right) 0^{\infty}$ (resp. closer to $0^{n-1}\left(l_{n} / 2\right) 0^{\infty}$ than to $\left.0^{\infty}\right)$. Set $J_{n}=\left\{0^{\infty}\right\}$. Property 1 in Definition 4.4 is obviously satisfied.

For each $n$, let $\pi_{n}(\Gamma)$ be the projection of $\Gamma$ at level $n$, that is the group defined naturally by the marked Schreier graph $\mathcal{S}_{n}$. Consider the abelian group $<a_{n+1}>\times<b_{n+1}>$ with generators $a_{n+1}, b_{n+1}$ where $a_{n+1}$ has order $l_{n+1}$ and $b_{n+1}$ has the same order as $\beta_{n+1}$ (possibly, infinity). Set

$$
\Gamma^{n+1}=\left[<a_{n+1}>\times<b_{n+1}>\right] \imath_{\mathcal{S}_{n}} \pi_{n}(\Gamma) .
$$

Let $\omega$ be a reduced word in $s_{1}=\alpha, s_{2}=\beta$ (an element in $\mathbf{F}=\langle\alpha>*<$ $\beta>$ ) which belong to $\Omega\left(J_{n}, B_{n}\right)$. By construction, its projection $g \in \Gamma$ has the form $\left(g_{x}\right)_{x \in \mathbb{T}^{n}} \sigma$ where $\sigma=\pi_{n}(g) \in \pi_{n}(\Gamma)$. Further, for all $x \in B_{n}, g_{x}$ is a power of $\beta_{n+1}$, for all $x \in \bar{B}_{n}, g_{x}$ is a power of $\alpha_{n+1}$ and for all $x \notin B_{n} \cup \bar{B}_{n}, g_{x}=1$. For $\omega \in \Omega\left(J_{n}, B_{n}\right)$, set

$$
\vartheta_{n}(\omega)=\left(\left(\tilde{g}_{x}\right)_{x \in \mathcal{S}_{n}}, \sigma\right) \text { with } \tilde{g}_{x}= \begin{cases}b_{n+1}^{q} & \text { if } x \in B_{n} \text { and } g_{x}=\beta_{n+1}^{q} \\ a_{n+1}^{q} & \text { if } x \in B_{n} \text { and } g_{x}=\alpha_{n+1}^{q}\end{cases}
$$

By inspection and the definition of $\Omega\left(J_{n}, B_{n}\right)$, if $\omega_{1}, \omega_{2}$ and $\omega_{1} \omega_{2}$ are all in $\Omega\left(J_{n}, B_{n}\right)$, we have $\left.\vartheta_{n}\left(\omega_{1}, \omega_{2}\right)=\vartheta_{n}\left(\omega_{1}\right) \vartheta_{n}(\omega) 2\right)$ as desired.

Having verified that assumption $(\Omega)$ holds, it remains to construct test functions $F_{n}, \psi_{n}$ as in Section 4.3

Set

$$
\psi_{n}\left(\left(\left(\tilde{g}_{x}\right)_{x \in \mathcal{S}_{n}}, \sigma\right)\right)=\prod_{x \in \mathcal{S}_{n}} \mathbf{1}_{[-r, r]^{2}}\left(\tilde{g}_{x}\right) .
$$

Here $r$ is a parameter to be specified later and $[-r, r]^{2}$ is understood as the set

$$
\left\{a_{n+1}^{-r}, \ldots, a_{n+1}^{r}\right\} \times\left\{b_{n+1}^{-r}, \ldots, b_{n+1}^{r}\right\}
$$

in $\left.\left\langle a_{n+1}\right\rangle \times<b_{n+1}\right\rangle$. Obviously,

$$
Q_{\Gamma^{n}, \mu}\left(\psi_{n}\right) \lesssim r^{-2}
$$

The needed $\left(J_{n}, B_{n}\right)$-admissible function $F_{n}$ on finite subsets of $\mathcal{S}$ is provided by Lemma A.4 and we have

$$
Q_{\mathcal{P}_{f}(\mathcal{S}), \mu}\left(F_{n}\right) \lesssim \frac{1}{\mathcal{R}\left(J_{n}, B_{n}^{c}\right)}
$$

Here, we have $\mathcal{R}\left(J_{n}, \mathcal{S}_{n}^{c}\right)=\sum_{1}^{n} 2^{n-j}\left(l_{j} / 4\right)$ and

$$
\mathcal{R}\left(J_{n}, B_{n}^{c}\right)=\mathcal{R}\left(J_{n}, \mathcal{S}_{n-1}^{c}\right)+\left\lfloor l_{n} / 8\right\rfloor \asymp \mathcal{R}\left(J_{n}, \mathcal{S}_{n}^{c}\right) .
$$

Picking $r \asymp \sqrt{\mathcal{R}\left(J_{n}, \mathcal{S}_{n}^{c}\right)}$ and applying Proposition 4.11 
Proof of Proposition 6.11. To prove this proposition, we will estimate $\Lambda_{2, \Gamma, \mu}$ and later $\Lambda_{2, \mathbb{Z} \imath_{\mathcal{S}} \Gamma, \mu}$ using the random walk on the right driven by $\mu$. Here $\mu$ is the uniform probability on $\left\{\alpha^{ \pm 1}, \beta^{ \pm 1}\right\}$.

We first explain how to prove the bound

$$
\Lambda_{2, \Gamma, \mu}(v) \leq \frac{C}{r^{2}} \text { for any } v \geq \exp \left(C V_{n-1} r \log r\right)
$$

where $r \in\left(0, l_{n} / 4\right)$ and $V_{n-1}=l_{1} \ldots l_{n-1}$ (the statement of Proposition 6.11 is for $\mathbb{Z} \imath_{\mathcal{S}} \Gamma$ instead of $\Gamma$ itself).

We embed $\Gamma$ in a larger group and use remark 2.4. Using the wreath recursion to level $n-1$ in $\operatorname{Aut}(\mathcal{T})$, we embed $\Gamma$ in

$$
\widetilde{\Gamma}=\Gamma_{n} \imath_{\mathcal{S}_{n-1}} \pi_{n-1}(\Gamma) .
$$

Recall that $\Gamma_{n}=<\alpha_{n}, \beta_{n}>$ and $\pi_{n-1}(\Gamma)$ is the finite group corresponding to the projection of $\Gamma$ acting on the finite subtree up to level $n-1$. We construct a test function $\Phi$ on $\widetilde{\Gamma}$.

Namely, any element of $g \in \widetilde{\Gamma}$ can be written as

$$
g=\left(g_{x}\right)_{x \in \mathcal{S}_{n-1}} \sigma
$$

with $g_{x} \in \Gamma_{n}$ and $\sigma \in \pi_{n-1}(\Gamma)$. Pick a test function $\phi_{r}$ defined on $\Gamma_{n}$ and set

$$
\Phi(g)=\prod_{\mathcal{S}_{n-1}} \phi_{r}\left(g_{x}\right) .
$$

We have

$$
g \alpha=\left(g_{x}\right)_{x \in \mathcal{S}_{n-1}} \pi_{n-1} \sigma(\alpha)
$$

and

$$
g \beta=\left(g_{x}^{\prime}\right)_{x \in \mathcal{S}_{n-1}} \sigma \pi_{n-1}(\beta)
$$

with $g_{x}^{\prime}=g_{x}$ except for $z_{1}=\sigma \cdot 0^{n-1}, z_{2}=\sigma \cdot 0^{n-2} y_{n-1}$ where $g_{z_{1}}^{\prime}=g_{z_{1}} \beta_{n}$ and $g_{z_{2}}^{\prime}=g_{z_{2}} \alpha_{n}$. If $\mu$ is the uniform measure on $\left\{\alpha^{ \pm 1}, \beta^{ \pm 1}\right\}$ and $\mu_{n}$ is the uniform measure on $\left\{\alpha_{n}^{ \pm 1}, \beta_{n}^{ \pm 1}\right\}$, this yields

$$
\frac{\mathcal{E}_{\widetilde{\Gamma}, \mu}^{r}(\Phi, \Phi)}{\|\Phi\|_{L^{2}(\widetilde{\Gamma})}^{2}} \leq 4 \frac{\mathcal{E}_{\Gamma_{n}, \mu_{n}}^{r}\left(\phi_{r}, \phi_{r}\right)}{\left\|\phi_{r}\right\|_{L^{2}\left(\Gamma_{n}\right)}^{2}} .
$$

Recall that our convention is that $\mathcal{E}^{r}$ is the Dirichlet form for the random walk on the right (The $r$ in $\mathcal{E}^{r}$ has nothing to do with the real parameter $r$ ).

We know little about $\Gamma_{n}$ whose structure is similar to that of $\Gamma$ but we have

$$
\Gamma_{n} \subset \Gamma_{n+1} \chi_{X_{n}} G_{n} .
$$

where $G_{n}$ is the cyclic group of order $l_{n}$ and $X_{n}=\left\{0, \ldots, l_{n}-1\right\}$ and every element in $\Gamma_{n}$ is of the form

$$
g=\left(g_{x}\right)_{x \in X_{n}} \alpha_{n}^{t}, \quad g_{x} \in \Gamma_{n+1}, t \in\left\{-l_{n}+1, \ldots, l_{n}-1\right\}
$$


where $\alpha_{n}$ is understood as either $\alpha_{n}$ itself or as the corresponding element in $G_{n}$.

Pick a parameter $r \in\left(0, l_{n} / 4\right)$ and set $\psi_{r}(m)=(1-|m| / r)_{+} . \operatorname{In} \prod_{x \in X_{n}}\left(\Gamma_{n+1}\right)_{x}$, consider the set $\Sigma_{r}$ parametrized by

$$
k_{x} \in\left\{-r^{2}, r^{2}\right\}, \quad x \in\{0, r\} \cup\left\{l_{n}-r-1, \ldots, l_{n}-1\right\} .
$$

of those elements of the form

$$
g_{x}=\left\{\begin{array}{cl}
\beta_{n+1}^{k_{x}} & \text { for } x \in\{0, r\} \cup\left\{l_{n}-r-1, \ldots, l_{n}-1\right\}, \\
k_{n+l_{n} / 2} & \text { for } x \in\left\{l_{n} / 2, l_{n} / 2+r\right\}, \\
\alpha_{n+1} & k_{x+l_{n} / 2} \\
\alpha_{n+1} & \text { for } x \in\left\{l_{n} / 2-r, l_{n} / 2-1\right\}, \\
e_{\Gamma_{n+1}} & \text { otherwise }
\end{array}\right.
$$

Set

$$
\phi_{r}(g)=\mathbf{1}_{\Sigma_{r}}\left(\left(g_{x}\right)_{X_{n}}\right) \psi_{r}(t), \quad g=\left(g_{x}\right)_{X_{n}} \alpha_{n}^{t} .
$$

Observe that for $g$ in the support of $\phi_{r}$,

$$
g \alpha_{n}^{ \pm 1}=\left(g_{x}\right)_{X_{n}} \alpha_{n}^{t \pm 1}
$$

and

$$
g \beta_{n}^{ \pm 1}=\left(g_{x}^{\prime}\right) \alpha_{n}^{t}
$$

where $g_{x}^{\prime}=g_{x}$ except at $z_{1}=\alpha_{n}^{t}(0)$ and $z_{2}=\alpha_{n}^{t}\left(l_{n} / 2\right)$. At this two locations, $g_{z_{1}}^{\prime}=\beta_{n+1}^{k_{z_{1}} \pm 1}$ and $g_{z_{2}}=\alpha_{n+1}^{k_{z_{1}} \pm 1}$. This implies that

$$
\mathcal{E}_{\Gamma_{n}, \mu_{n}}^{r}\left(\phi_{r}, \phi_{r}\right) \leq C r^{-2}\left\|\phi_{r}\right\|_{L^{2}\left(\Gamma_{n}\right)}^{2} .
$$

Moreover, when $r$ is an integer,

$$
\left|\operatorname{supp}\left(\phi_{r}\right)\right|=\left(2 r^{2}\right)^{2 r}(2 r-1) .
$$

Returning to the test function $\Phi$ on $\widetilde{\Gamma}=\Gamma_{n} \imath_{\mathcal{S}_{n-1}} \pi_{n-1}(\Gamma)$, we have

$$
\frac{\mathcal{E}_{\widetilde{\Gamma}, \mu}(\Phi, \Phi)}{\|\Phi\|_{L^{2}(\widetilde{\Gamma})}^{2}} \leq C r^{-2}
$$

and

$$
\begin{aligned}
|\operatorname{supp}(\Phi)| & =\left[\left(2 r^{2}\right)^{2 r}(2 r-1)\right]^{V_{n-1}}\left|\pi_{n}(\Gamma)\right| \\
& =\left[\left(2 r^{2}\right)^{2 r}(2 r-1)\right]^{V_{n-1}} \prod_{1}^{n-1}\left(l_{j}\right)^{l_{1} \ldots l_{j-1}} \\
& \leq \exp \left(C V_{n-1} r \log r\right)
\end{aligned}
$$

for $r \in\left(0, l_{n} / 4\right)$. This yields

$$
\Lambda_{2, \Gamma}(v) \leq \frac{C}{r^{2}} \text { for all } v \geq \exp \left(C V_{n-1} r \log r\right) \text { and } r \in\left(0, l_{n} / 4\right) .
$$


Next, we prove the similar result on $\mathbb{Z} \imath_{\mathcal{S}} \Gamma$. We observe that the test function $\Phi$ on $\widetilde{\Gamma} \supset \Gamma$ produces a test function $\Psi$ on $\Gamma$ itself with

$$
\frac{\mathcal{E}_{\Gamma, \mu}^{r}(\Psi, \Psi)}{\|\Psi\|_{L^{2}(\Gamma)}^{2}} \leq \frac{\mathcal{E}_{\widetilde{\Gamma}, \mu}^{r}(\Phi, \Phi)}{\|\Phi\|_{L^{2}(\widetilde{\Gamma})}^{2}} \leq \frac{C}{r^{2}}
$$

and, using the wreath recursion to level $n-1$, any $g \in \Gamma$ is of the form $\left(g_{x}\right)_{S_{n-1}} \sigma$ with $g_{x} \in \Gamma_{n}$ and $\sigma \in \pi_{n-1}(\Gamma)$ and

$$
\operatorname{supp}(\Psi) \subset\left\{g=\left(g_{x}\right)_{\mathcal{S}_{n-1}} \sigma: \phi_{r}\left(g_{x}\right) \neq 0, x \in \mathcal{S}_{n-1}\right\}
$$

For any $g \in \operatorname{supp}(\Psi)$, we have $g \cdot 0^{\infty} \in U_{n}(r)$ where $U_{n}(r)$ is described (see Figure 15D) using the tree indexing of the points in $\mathcal{S}$ as

$U_{n}(r)=\left\{x_{1} \ldots x_{n-1} y 0^{\infty}: x_{i} \in X_{i}, i \leq n-1, y \in\{0, \ldots, r\} \cup\left\{l_{n}-r, \ldots, l_{n}-1\right\}\right\}$.

Figure 15: Sketch of the set $U_{n}(r)$ (in black) with the root $o$ marked in red. Each little circle represents a copy of the graph $\mathcal{S}_{n-1}$.

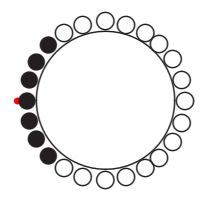

On $\mathbb{Z} \imath_{\mathcal{S}} \Gamma$, consider the test function

$$
\Phi^{\prime}((f, g))=\prod_{x \in U_{n}(r)} \mathbf{1}_{\left[-r^{2}, r^{2}\right]}(f(x)) \Psi(g) .
$$

On $\mathbb{Z} \imath_{\mathcal{S}} \Gamma$ and for $(f, g) \in \operatorname{supp}\left(\Phi^{\prime}\right)$, we have

$$
(f, g)\left(\mathbf{1}_{ \pm 1}^{0^{\infty}}, e_{\Gamma}\right)=\left(f \mathbf{1}_{ \pm 1}^{g \cdot 0^{\infty}}, g\right)
$$

and

$$
(f, g)\left(\mathbf{e}_{\mathbb{Z}}^{\mathcal{S}}, \gamma\right)=(f, g \gamma), \gamma \in \Gamma .
$$

This gives

$$
\mathcal{E}_{\mathbb{Z} \imath_{\mathcal{S}} \Gamma, \mathfrak{q}}^{r}\left(\Phi^{\prime}, \Phi^{\prime}\right) \leq \frac{C}{r^{2}}
$$

where $\mathfrak{q}=\frac{1}{2}(\nu+\mu)$ and $\nu$ is the uniform measure on \pm 1 . Finally,

$$
\left|\operatorname{supp}\left(\Phi^{\prime}\right)\right|=\left(1+2 r^{2}\right)^{\left|U_{N}(r)\right|}|\operatorname{supp}(\Psi)| \leq \exp \left(C V_{n-1} r \log r\right),
$$

with $0 \leq r \leq l_{n} / 4$ The desired bound follows. 


\subsection{Concrete examples of the type $\mathbb{Z} \imath_{\mathcal{S}} \Gamma$}

This section is devoted to spelling out examples that illustrate our results in the case $G=\mathbb{Z} \imath_{\mathcal{S}} \Gamma$ when $\Gamma$ belongs to the family of a cyclic Neumann-Segal groups associated with sequences $\left(l_{n}\right)_{1}^{\infty}$ of even integers that are growing fast enough.

Theorem 6.15. Assume that $l_{n}=2^{\kappa(n)}$ with $\kappa(n+1) \geq \kappa(n)+1$. Then, for $r \in\left[l_{n-1}, l_{n}\right]$, we have

$$
\Lambda_{1, \mathbb{Z} \imath_{\mathcal{S}} \Gamma}(v) \leq \frac{c}{r} \text { for } v \leq \exp \left(c V_{n-1} r \log r\right)
$$

and

$$
\Lambda_{2, Z_{\imath \mathcal{S}} \Gamma}(v) \leq \frac{C}{r^{2}} \text { for } v \geq \exp \left(C V_{n-1} r \log r\right)
$$

Equivalently,

$$
\Lambda_{1, \mathbb{Z}_{\imath_{\mathcal{S}} \Gamma}}^{2}(v) \asymp \Lambda_{2, \mathbb{Z} \imath_{\mathcal{S}} \Gamma}(v) \asymp \begin{cases}r^{-2} & \text { for } \log v=V_{n-1} r \log r, r \in\left[l_{n-1}, l_{n}\right] \\ l_{n}^{-2} & \text { for } \log v \in\left[V_{n-1} l_{n} \log l_{n}, V_{n} l_{n} \log l_{n}\right] .\end{cases}
$$

Proof. This follows from Corollary 6.2 and Proposition 6.11.

Corollary 6.16. Assume that $l_{n}=2^{\kappa(n)}$ with $\kappa(n+1) \geq \kappa(n)+1$. The random walk invariant $\Phi_{\mathbb{Z}{ }_{\mathcal{S}} \Gamma}$ is given as follows:

- For $t \in\left[V_{n-1} l_{n-1}^{3} \log l_{n-1}, V_{n-1} l_{n}^{3} \log l_{n}\right]$,

$$
-\log \left(\Phi_{\mathbb{Z} \imath_{\mathcal{S}} \Gamma}(t)\right) \asymp V_{n-1}^{2 / 3} t^{1 / 3}\left(\log \left(t / V_{n-1}\right)\right)^{2 / 3} .
$$

- For $t \in\left[V_{n-1} l_{n}^{3} \log l_{n}, V_{n} l_{n}^{3} \log l_{n}\right]$,

$$
-\log \left(\Phi_{\mathbb{Z} \imath_{\mathcal{S}} \Gamma}(t)\right) \asymp t / l_{n}^{2} .
$$

Proof. This follows by (somewhat lengthy) inspection from the previous theorem and the well-know relations between $\Lambda_{2}$ and $\Phi$ See, e.g., 4, 27] and the references therein.

Corollary 6.16 gives a complete picture of the behavior of the probability of return for simple random on the cyclic Neumann-Segal groups $\Gamma$ considered here when $l_{n}=2^{\kappa(n)}$ with $\kappa$ growing at least linearly. The result provides a continuum of distinct explicit behaviors for the random walk invariant $\Phi_{G}$ as well as for the profiles $\Lambda_{1, G}, \Lambda_{2, G}$.

Remark 6.17. Theorem 6.15 and Corollary 6.16 and the examples described below are all concerned with the group $G=\mathbb{Z}_{\imath_{\mathcal{S}}} \Gamma$ and not with $\Gamma$ itself. Since $\Gamma$ is a subgroup of $G$ we have $\Lambda_{1, \Gamma} \leq \Lambda_{1, G}, \Lambda_{2, \Gamma} \leq \Lambda_{2, G}$ and $-\log \Phi_{\Gamma} \lesssim-\log \Phi_{G}$.

Under the hypotheses of Theorem 6.15] and Corollary 6.16, we also have that

$$
\Lambda_{1, \Gamma}(v) \geq \frac{c}{l_{n}} \text { for } \log v \in\left[V_{n-1} l_{n}, V_{n} l_{n}\right]
$$


On the interval $\left[V_{n-1} l_{n_{1}}, V_{n-1} l_{n}\right]$ which is not covered by these estimates, we have no better lower bounds than those obtained by monotonicity or by using the classical volume bound of [9] together with Lemma 6.1] This means that, in general, we are not able to provide matching two-sided bounds for the isoperimetric profiles of the group $\Gamma$ itself.

Nevertheless, there are cases where using monotonicity is sufficient to obtain a satisfactory result such as in Theorem 6.14 where $l_{n}=2^{1+\left\lfloor n^{\gamma}\right\rfloor}$, with $\gamma>1$. Even in this case, Theorem 6.15 and Corollary 6.16 gives more precise results on $\mathbb{Z} \imath_{\mathcal{S}} \Gamma$ than what we know for $\Gamma$. Namely, a careful application of Theorem 6.15 and Corollary 6.16 show that, when $l_{n}=2^{1+\left\lfloor n^{\gamma}\right\rfloor}$ with $\gamma>1$, we have

$$
\Lambda_{1, \Gamma}(v)^{2} \asymp \Lambda_{2, \Gamma}(v) \asymp\left(\frac{2^{\left[(1+\gamma) \log _{2} \log v\right]^{\gamma /(1+\gamma)}}}{\log v}\right)^{2} .
$$

and

$$
-\log \left(\Phi_{\mathbb{Z} \imath_{S} \Gamma}(t)\right) \asymp \frac{t}{2^{2\left((1+\gamma) \log _{2} t\right)^{\gamma /(1+\gamma)}}} .
$$

Compare with Theorem 6.14.

The following examples illustrate what happen when $\kappa$ grows faster than $2^{1+\left\lfloor n^{\gamma}\right\rfloor}, \gamma>1$. In each of these examples, we describe the extreme behaviors of the function $-\log \left(\Phi_{\mathbb{Z}_{\mathcal{S}} \Gamma}\right)$ which, according to Corollary 6.16] are obtained at the points $t=V_{n-1} l_{n}^{3} \log l_{n}$ and $t=V_{n} l_{n}^{3} \log l_{n}$. Note that Corollary 6.16 provides complementary sharp estimates at all times.

Example 6.18. Let $l_{n}=2^{\varkappa^{n}}$, that is $\kappa(n)=\varkappa^{n}, \varkappa>1$. In this case,

$$
\log _{2} V_{n}=\frac{\varkappa}{\varkappa-1}\left(\varkappa^{n}-1\right), \quad V_{n-1} l_{n}^{3} \asymp l_{n}^{\frac{3 \varkappa-1}{\varkappa-1}}, \quad V_{n} l_{n}^{3} \asymp l_{n}^{\frac{4 \varkappa-3}{\varkappa-1}} .
$$

This gives:

- For $t \asymp V_{n-1} l_{n}^{3} \log l_{n} \asymp l_{n}^{\frac{3 \varkappa-2}{\varkappa-1}} \log l_{n}$,

$$
-\log \left(\Phi_{\mathbb{Z}_{\mathcal{S}} \Gamma}(t)\right) \asymp t^{\frac{\varkappa}{3 \varkappa-2}}(\log t)^{\frac{2 \kappa-2}{3 \kappa-2}}
$$

- For $t \asymp V_{n} l_{n}^{3} \log l_{n} \asymp l_{n}^{\frac{4 \varkappa-1}{\varkappa-1}} \log l_{n}$,

$$
-\log \left(\Phi_{\mathbb{Z}_{\mathcal{S}} \Gamma}(t)\right) \asymp t^{\frac{2 \varkappa-1}{4 \varkappa-3}}(\log t)^{\frac{2 \kappa-2}{4 \kappa-3}} .
$$

In addition, the corollary also gives that, for all $t>1$,

$$
c t^{\frac{\varkappa}{\varkappa \varkappa-2}}(\log t)^{\frac{2 \kappa-2}{3 \kappa-2}} \leq-\log \left(\Phi_{\mathbb{Z}_{\mathcal{S}} \Gamma}(t)\right) \leq C t^{\frac{2 \varkappa-1}{4 \varkappa-3}}(\log t)^{\frac{2 \kappa-2}{4 \kappa-3}} .
$$

Example 6.19. Let $l_{n}$ be such that $l_{n}=2^{V_{n-1}}$. In this case,

$$
V_{n-1} l_{n}^{3} \log l_{n} \asymp V_{n-1}^{2} 2^{3 V_{n-1}}, \quad V_{n} l_{n}^{3} \log l_{n} \asymp V_{n-1}^{2} 2^{4 V_{n-1}} .
$$

This gives: 
- For $t \asymp V_{n-1} l_{n}^{3} \log l_{n} \asymp V_{n-1}^{2} 2^{3 V_{n-1}}$,

$$
-\log \left(\Phi_{\mathbb{Z} \imath_{\mathcal{S}} \Gamma}(t)\right) \asymp t^{\frac{1}{3}}(\log t)^{\frac{4}{3}} ;
$$

- For $t \asymp V_{n} l_{n}^{3} \log l_{n} \asymp V_{n-1}^{2} 2^{4 V_{n-1}}$,

$$
-\log \left(\Phi_{\mathbb{Z} l_{S} \Gamma}(t)\right) \asymp t^{\frac{1}{2}} \log t .
$$

Further, for all $t>1$, we have

$$
c t^{\frac{1}{3}}(\log t)^{\frac{4}{3}} \leq-\log \left(\Phi_{\mathbb{Z} \imath_{S} \Gamma}(t)\right) \leq C t^{\frac{1}{2}} \log t .
$$

Example 6.20. Suppose now we have a sequence of integer $n_{i}$ tending to infinity such that

$l_{n_{i}}=2^{V_{n_{i}-1}}, n_{i+1}=V_{n_{i}-1}+n_{i}+1$ and $l_{n_{i}+j}=2 l_{n_{i}+j-1}, 1 \leq j \leq n_{i+1}-n_{i}-1$.

On the one hand, at time $t_{i} \asymp V_{n_{i}-1} l_{n_{i}}^{3} \log l_{n_{i}}$, by the same computation as in the previous example, we have

$$
-\log \Phi_{\mathbb{Z}_{\mathcal{S}} \Gamma}\left(t_{i}\right) \asymp t_{i}^{\frac{1}{3}}\left(\log t_{i}\right)^{\frac{4}{3}} .
$$

On the other hand,

$$
\begin{aligned}
V_{n_{i+1}-1}=V_{n_{i}-1} l_{n_{i}} \ldots l_{n_{i+1}-1} & =2^{\left(n_{i+1}-n_{i}\right) V_{n_{i}-1}+\sum_{1}^{n_{i+1}-n_{i}-1}{ }^{j}} V_{n_{i}-1} \\
& =2^{\frac{3}{2}\left(V_{n_{i}-1}^{2}+V_{n_{i}-1}\right)} V_{n_{i}-1}, \\
l_{n_{i+1}-1} & =2^{2 V_{n_{i}-1}} .
\end{aligned}
$$

Hence, at time

$$
t_{i}^{\prime} \asymp V_{n_{i}-1}^{2} 2^{\frac{3}{2}\left(V_{n_{i}-1}^{2}+\frac{15}{2} V_{n_{i}-1}\right)}
$$

we have

$$
\begin{aligned}
-\log \Phi_{\mathbb{Z} \mathfrak{s} \Gamma}\left(t_{i}^{\prime}\right) & \asymp V_{n_{i}-1}^{2} 2^{\frac{3}{2}\left(V_{n_{i}-1}^{2}+\frac{7}{2} V_{n_{i}-1}\right)} \\
& \asymp t_{i}^{\prime} 2^{-4 V_{n_{i}-1}} \asymp \frac{t_{i}^{\prime}}{2^{4\left(\frac{2}{3} \log _{2} t_{i}^{\prime}\right)^{1 / 2}}} .
\end{aligned}
$$

Also, for all $t>1$, we have

$$
c t^{\frac{1}{3}}(\log t)^{\frac{4}{3}} \leq-\log \left(\Phi_{\mathbb{Z}_{\mathcal{S}} \Gamma}(t)\right) \leq C \frac{t}{2^{4\left(\frac{2}{3} \log _{2} t\right)^{1 / 2}}} .
$$

Example 6.21. Suppose now we have a sequence of integer $n_{i}$ tending to infinity such that

$$
l_{n_{i}}=V_{n_{i}-1}^{\varkappa-1}, n_{i+1}=\log _{2} V_{n_{i}-1}^{\varkappa-1}+n_{i}+1 \text { and } l_{n_{i}+j}=2 l_{n_{i}+j-1}, 1 \leq j \leq n_{i+1}-n_{i}-1,
$$


for some $\varkappa>1$. By the same computation as in Example ??, we have

$$
-\log \Phi_{\mathbb{Z} \imath_{\mathcal{S}} \Gamma}\left(t_{i}\right) \asymp t_{i}^{\frac{\varkappa}{3 \varkappa-2}}\left(\log t_{i}\right)^{\frac{2 \varkappa-2}{3 \varkappa-2}} .
$$

On the other hand, if we set $N_{i}=n_{i+1}-n_{i}-1=\log _{2}\left(V_{n_{i}-1}^{\varkappa-1}\right)$,

$$
\begin{gathered}
V_{n_{i+1}-1}=V_{n_{i}-1} l_{n_{i}} \ldots l_{n_{i+1}-1}=V_{n_{i}-1}^{1+(\varkappa-1)\left(N_{i}+1\right)} 2^{\frac{1}{2} N_{i}\left(N_{i}+1\right)} \\
=2^{\frac{3}{2} N_{i}^{2}+\left(\frac{1}{2}+\frac{\varkappa}{\varkappa-1}\right) N_{i}} \\
l_{n_{i+1}-1}=V_{n_{i}-1}^{\varkappa-1} 2^{N_{i}}=2^{2 N_{i}}
\end{gathered}
$$

Hence, at time

$$
t_{i}^{\prime} \asymp V_{n_{i+1}-1} l_{n_{i+1}-1}^{3} \log l_{n_{i+1}-1} \asymp 2 N_{i} 2^{\frac{3}{2} N_{i}^{2}+\left(6+\frac{1}{2}+\frac{\varkappa}{\varkappa-1}\right) N_{i}},
$$

we have

$$
-\log \Phi_{\mathbb{Z} l_{\mathcal{S}} \Gamma}\left(t_{i}^{\prime}\right) \asymp \frac{t_{i}^{\prime}}{2^{-4 N_{i}}} \asymp \frac{t_{i}^{\prime}}{2^{4\left(\frac{2}{3} \log t_{i}^{\prime}\right)^{1 / 2}}} .
$$

In addition, Corollary 6.16 also gives that, for all $t>1$,

$$
c t^{\frac{\varkappa}{3 \varkappa-2}}(\log t)^{\frac{2 \varkappa-2}{3 \varkappa-2}} \leq-\log \left(\Phi_{\mathbb{Z} l_{\mathcal{S}} \Gamma}(t)\right) \leq C \frac{t}{2^{4\left(\frac{2}{3} \log t\right)^{1 / 2}}} .
$$

\section{A Appendix: action on finite sets}

As in the core of the paper, let $\Gamma$ be a finitely generated group, with generating set $S$, acting on space $X$ with a reference point $o$ chosen in $X$. Let $\mathcal{S}$ denote the orbital Schreier graph of $o$ under the action of $G$. Let $\mu$ be a symmetric probability measure on $\Gamma$. We are concern here with Consider the action of $\Gamma$ on $\mathcal{P}_{f}(\mathcal{S})=\oplus_{\mathcal{S}} \mathbb{Z}_{2}$.

Definition A.1. Let $J, B$ be fixed finite subsets of $\mathcal{S}$ and $X$, respectively, with $J \subset B$. Set

$$
L^{2}\left(\mathcal{P}_{f}(X) ; J ; B\right)=\left\{\Psi \in L^{2}\left(\mathcal{P}_{f}(X)\right): A \in \operatorname{supp} \Psi \Rightarrow J \subseteq A \subseteq B\right\}
$$

and

$$
\lambda_{\mathcal{P}_{f}(\mathcal{S}), \mu}(B ; J)=\inf \left\{\frac{\sum \mu(s)\|s \cdot \Psi-\Psi\|_{2}^{2}}{\|\Psi\|_{2}^{2}}: 0 \neq \Psi \in L^{2}\left(\mathcal{P}_{f}(X) ; J ; B\right)\right\} .
$$

Here as usual the action of $\Gamma$ on functions is given by $(g \cdot F)(A)=F\left(g^{-1} \cdot A\right)$, $A \in \mathcal{P}_{f}(X)$. The requirement that $J \subset A$ for every $A$ in the supp $\Psi$ needs some justification. If, instead, we look at

$$
\inf \left\{\frac{\sum \mu(s)\|s \cdot F-F\|_{L^{2}\left(\mathcal{P}_{f}(X)\right)}^{2}}{\|F\|_{L^{2}\left(\mathcal{P}_{f}(X)\right)}^{2}}: \operatorname{supp} F \subseteq\{0,1\}^{B}\right\}
$$


then it agrees with the usual notion of Markov chain spectral gap (with Dirichlet boundary condition) on $B$. But this infimum is 0 because we can take the function $F=1$ on the empty set (all 0 configuration), and $F=0$ everywhere else. The important additional requirement is that every set in the support of $\Psi$ to contain a specific set $J$. This requirement is also justified by the fact that the action of $\mathbb{Z}_{2} l_{X} \Gamma$ on $\mathcal{P}_{f}(X)$ is amenable if and only if the action of $\Gamma$ on $\mathcal{P}_{f}(X)$ admits an invariant mean giving full weight to the collection of sets containing a fixed finite set, see [19, Lemma 3.1]. In the context of Section 4.1] $J=J_{n}$ is chosen as a set of "special points" which have the property that having control over their inverted orbit implies the existence of the local embedding $\vartheta_{n}$ in Definition 4.4 .

We now describe an upper bound on $\lambda_{\mathcal{P}_{f}(\mathcal{S}), \mu}(B ; J)$ based on arguments inspired by [20]. Here the notions of energy of functions on the graph $\mathcal{E}_{\mathcal{S}, \mu}$, resistance $\mathcal{R}_{\mathcal{S}, \mu}(U \leftrightarrow V)$ are all standard. Namely,

$$
\mathcal{E}_{\mathcal{S}, \mu}(h, h)=\frac{1}{2} \sum_{g \in \Gamma} \sum_{x \in \mathcal{S}}|h(x)-h(g \cdot x)|^{2} \mu(g),
$$

and, for $U \subset V \subset \mathcal{S}$,

$$
\mathcal{R}_{\mathcal{S}, \mu}(U \leftrightarrow V)^{-1}=\inf \left\{\mathcal{E}_{\mathcal{S}, \mu}(h, h): h=1 \text { on } U, h=0 \text { on } V\right\} .
$$

Lemma A.2 (Compare to part of [20, Theorem 2.8]). Fix finite subsets $J \subset$ $B \subset \mathcal{S}$. Given a function $h: X \rightarrow[0,1]$ such that $h=1$ on $J, h=0$ on $B^{c}$ and $\mathcal{E}_{\mathcal{S}, \mu}(h, h) \leq 1 / 2$, there exists a function $F_{h}: \mathcal{P}_{f}(X) \rightarrow[0,1]$ such that $\left\|F_{h}\right\|_{L^{2}\left(\mathcal{P}_{f}(X)\right)}^{2}=1, A \in \operatorname{supp}\left(F_{h}\right) \Rightarrow J \subseteq A \subseteq B$ and

$$
\sum_{s \in \Gamma} \mu(s)\left\|s \cdot F_{h}-F_{h}\right\|_{L^{2}\left(\mathcal{P}_{f}(X)\right)}^{2} \leq \frac{\pi^{2}}{2} \mathcal{E}_{\mathcal{S}, \mu}(h, h) .
$$

In particular, we have

$$
\lambda_{\mathcal{P}_{f}(\mathcal{S}), \mu}(J ; B) \leq \frac{\pi^{2} / 2}{\mathcal{R}_{\mathcal{S}, \mu}\left(J \leftrightarrow B^{c}\right)} .
$$

Proof. Given a function $h: X \rightarrow[0,1]$, define $F_{h}: \mathcal{P}_{f}(X) \rightarrow[0,1]$ by setting

$$
F_{h}(\eta):=\prod_{v \in X} \xi_{h(v)}(\eta(v)), \quad \eta \in \mathcal{P}_{f}(X)=\oplus_{X} \mathbb{Z}_{2}
$$

where

$$
\xi_{a}(0):=\cos \left(\frac{\pi a}{2}\right), \xi_{a}(1):=\sin \left(\frac{\pi a}{2}\right) .
$$

Note that if $\eta(u)=0$ for some $u \in J$, we must have $h(u)=1$ and it follows that $\xi_{h(u)}(\eta(u))=\xi_{1}(0)=\cos (\pi / 2)=0$. Therefore $A \in \operatorname{supp}\left(F_{h}\right) \Rightarrow J \subseteq A$. Similarly, if $\eta(v)=1$ for some $v \in B^{c}$ then $h(v)=0, \xi_{h(v)}(\eta(v))=\xi_{0}(1)=$ $\sin (0)=0$. In particular, we have $\operatorname{supp}\left(F_{h}\right) \subseteq\{0,1\}^{B}$. 
To compute the relevant $L^{2}$ norms, write

$$
\begin{aligned}
\left\|F_{h}\right\|_{L^{2}\left(\mathcal{P}_{f}(X)\right)}^{2} & =\sum_{\eta} F_{h}(\eta)^{2}=\sum_{\eta} \prod_{v \in X} \xi_{h(v)}(\eta(v))^{2} \\
& =\prod_{v \in \Omega}\left[\xi_{h(v)}(1)^{2}+\xi_{h(v)}(0)^{2}\right]=1
\end{aligned}
$$

and

$$
\left\|s \cdot F_{h}-F_{h}\right\|_{L^{2}\left(\mathcal{P}_{f}(X)\right)}^{2}=2\left\|F_{h}\right\|_{L^{2}\left(\mathcal{P}_{f}(X)\right)}^{2}-2\left\langle s \cdot F_{h}, F_{h}\right\rangle_{L^{2}\left(\mathcal{P}_{f}(X)\right)}^{2} .
$$

Note that

$$
\begin{aligned}
s \cdot F_{h}(\eta) & =F_{h}\left(s^{-1} \cdot \eta\right)=\prod_{v \in X} \xi_{h(v)}\left(s^{-1} \cdot \eta(v)\right) \\
& =\prod_{v \in X} \xi_{h(v)}(\eta(s \cdot v))=\prod_{v \in X} \xi_{h\left(s^{-1} \cdot v\right)}(\eta(v)) .
\end{aligned}
$$

Therefore, we have

$$
\begin{aligned}
\left\langle s \cdot F_{h}\right. & \left., F_{h}\right\rangle=\sum_{\eta} F_{h}\left(s^{-1} \cdot \eta\right) \cdot F_{h}(\eta) \\
& =\sum_{\eta} \prod_{v} \xi_{h\left(s^{-1} \cdot v\right)}(\eta(v)) \xi_{h(v)}(\eta(v)) \\
& =\prod_{v \in B}\left[\cos \left(\frac{\pi}{2} h\left(s^{-1} \cdot v\right)\right) \cos \left(\frac{\pi}{2} h(v)\right)+\sin \left(\frac{\pi}{2} h\left(s^{-1} \cdot v\right)\right) \sin \left(\frac{\pi}{2} h(v)\right)\right] \\
& =\prod_{v \in B} \cos \left(\frac{\pi}{2}\left(h\left(s^{-1} \cdot v\right)-h(v)\right)\right) .
\end{aligned}
$$

Now, use the fact that $\cos (x) \geq e^{-x^{2}}$ if $|x| \leq \pi / 4$, together with the assumption that $\|s \cdot h-h\|_{L^{2}(X)}^{2} \leq \frac{1}{2}$, to obtain

$$
\begin{aligned}
\prod_{v \in \Omega} \cos \left(\frac{\pi}{2}\left(h\left(s^{-1} \cdot v\right)-h(v)\right)\right) & \geq \prod_{v \in G} \exp \left(-\frac{\pi^{2}}{4}\left(h\left(s^{-1} \cdot v\right)-h(v)\right)^{2}\right) \\
& =\exp \left(-\frac{\pi^{2}}{4}\|s \cdot h-h\|_{L^{2}(X)}^{2}\right) \\
& \geq 1-\frac{\pi^{2}}{4}\|s \cdot h-h\|_{L^{2}(X)}^{2} .
\end{aligned}
$$

We conclude that

$$
\sum_{s \in \Gamma} \mu(s)\left\|s \cdot F_{h}-F_{h}\right\|_{L^{2}\left(\mathcal{P}_{f}(X)\right)}^{2} \leq \frac{\pi^{2}}{2} \mathcal{E}_{\mathcal{S}, \mu}(h, h) .
$$


Remark A.3. The function $F_{h}$ is a product of functions at each point in $X$ and the previous computation does not involve information about the relations between different orbits. Asking for a better function is related to the problem of finding a better method than using an union bound. As the action of $\Gamma$ on subsets of $X$ is usually quite intricate, it is a rather difficult question. But in simple cases such as the dihedral group (Subsection ??) and the bubble groups (Subsection ??), one can find a better function by inspecting how certain subsets move under the group action.

Lemma A.4. Let $(\Gamma, X, o)$ and $J_{n}, B_{n}$ be as in Definition 4.4. For each $n$, there exists a $\left(J_{n}, B_{n}\right)$-admissible function $F_{n}$ such that

$$
\mathcal{Q}_{\mathcal{P}(\mathcal{S}), \mu}\left(F_{n}\right) \leq \frac{\pi^{2} / 2}{\mathcal{R}_{\mathcal{S}, \mu}\left(J_{n} \leftrightarrow B_{n}^{c}\right)} .
$$

Proof. To obtain a function $F_{n}$ that is $\left(J_{n}, B_{n}\right)$-admissible as required in 4.1 and which has small Rayleigh quotient, consider the function $F_{n}^{*}=F_{h}$ of Lemma A.2 associated with $J_{n}, B_{n}$ and the corresponding optimal choice of $h$ so that $\mathcal{E}_{\mathcal{S}, \mu}(h, h)=\mathcal{R}_{\mathcal{S}, \mu}\left(J_{n} \leftrightarrow B_{n}^{c}\right)^{-1}$. Observe that the support of $F_{n}^{*}$ can be partitioned into orbits of certain finite subsets of $B_{n}$. In particular, there must exists a finite subset $A_{n}$ such that the restriction of $F_{n}^{*}$ to the orbit of $A_{n}$ has Rayleigh quotient bounded above by $\frac{\pi^{2}}{2} \mathcal{R}_{\mathcal{S}, \mu}\left(J_{n} \leftrightarrow B_{n}^{c}\right)^{-1}$.

In particular, the function

$$
F_{n}(Y)=\left\{\begin{array}{cl}
F_{n}^{*}(Y) & \text { if } Y=g \cdot A_{n} \text { for some } g \in G \\
0 & \text { otherwise }
\end{array}\right.
$$

is $\left(J_{n}, B_{n}\right)$-admissible and satisfies

$$
\mathcal{Q}_{\mathcal{P}_{f}(\mathcal{S}), \mu}\left(F_{n}\right) \leq \frac{\pi^{2} / 2}{\mathcal{R}_{\mu}\left(J_{n} \leftrightarrow B_{n}^{c}\right)}
$$

as desired.

\section{References}

[1] Gideon Amir, Omer Angel, and Bálint Virág. Amenability of linearactivity automaton groups. Journal of the European Mathematical Society, 15.3:705-730, 2013.

[2] Laurent Bartholdi and Anna Erschler. Growth of permutational extensions. Inventiones Mathematicae, 189(2):431-455, 2012.

[3] Laurent Bartholdi and Floriane Pochon. On growth and torsion of groups. Groups Geom. Dyn., 3(4):525-539, 2009.

[4] Alexander Bendikov, Christophe Pittet, and Roman Sauer. Spectral distribution and $L^{2}$-isoperimetric profile of Laplace operators on groups. Math. Ann., 354(1):43-72, 2012. 
[5] Ievgen V. Bondarenko. Growth of Schreier graphs of automaton groups. Math. Ann., 354(2):765-785, 2012.

[6] J. Brieussel. Behaviors of entropy on finitely generated groups. 2011.

[7] Jérémie Brieussel. Amenability and non-uniform growth of some directed automorphism groups of a rooted tree. Math. Z., 263(2):265-293, 2009.

[8] Thierry Coulhon. Ultracontractivity and Nash type inequalities. J. Funct. Anal., 141(2):510-539, 1996.

[9] Thierry Coulhon and Laurent Saloff-Coste. Isopérimétrie pour les groupes et les variétés. Rev. Mat. Iberoamericana, 9(2):293-314, 1993.

[10] Anna Erschler. On isoperimetric profiles of finitely generated groups. Geom. Dedicata, 100:157-171, 2003.

[11] Anna Erschler. Isoperimetry for wreath products of Markov chains and multiplicity of selfintersections of random walks. Probab. Theory Related Fields, 136(4):560-586, 2006.

[12] Anna Erschler. Piecewise automatic groups. Duke Math. J., 134(3):591613, 2006.

[13] Jacek Fabrykowski and Narain Gupta. On groups with sub-exponential growth functions. J. Indian Math. Soc. (N.S.), 49(3-4):249-256 (1987), 1985.

[14] Elisabeth Fink. A finitely generated branch group of exponential growth without free subgroups. Journal of Algebra, 397:625-642, 2014.

[15] Rostislav I Grigorchuk. Just infinite branch groups. In New horizons in pro-p groups, pages 121-179. Springer, 2000.

[16] Rostislav I Grigorchuk. Some topics in the dynamics of group actions on rooted trees. Proceedings of the Steklov Institute of Mathematics, 273(1):64$175,2011$.

[17] L. H. Harper. Global methods for combinatorial isoperimetric problems, volume 90 of Cambridge Studies in Advanced Mathematics. Cambridge University Press, Cambridge, 2004.

[18] Kate Juschenko, Nicols Matte Bon, Nicolas Monod, and Mikael de la Salle. Extensive amenability and an application to interval exchanges. ArXiv e-prints, March 2015.

[19] Kate Juschenko and Nicolas Monod. Cantor systems, piecewise translations and simple amenable groups. Annals of Mathematics, 178(EPFLARTICLE-179598):775-787, 2013. 
[20] Kate Juschenko, Volodymyr Nekrashevych, and Mikael de la Salle. Extensions of amenable groups by recurrent groupoids. arXiv preprint arXiv:1305.2637, 2013.

[21] Michał Kotowski and Bálint Virág. Non-Liouville groups with return probability exponent at most 1/2. arXiv preprint arXiv:1408.6895, 2014.

[22] R. Lyons and Y. Peres. Probability on Trees and Networks. Cambridge Studies in Advanced Mathematics. Cambridge University Press, Cambridge, 2016. To appear.

[23] Peter M. Neumann. Some questions of Edjvet and Pride about infinite groups. Illinois J. Math., 30(2):301-316, 1986.

[24] C. Pittet and L. Saloff-Coste. On random walks on wreath products. Ann. Probab., 30, no.2:948-977, 2002.

[25] Ch. Pittet and L. Saloff-Coste. On the stability of the behavior of random walks on groups. J. Geom. Anal., 10(4):713-737, 2000.

[26] Laurent Saloff-Coste. Probability on groups: random walks and invariant diffusions. Notices Amer. Math. Soc., 48(9):968-977, 2001.

[27] Laurent Saloff-Coste and Tianyi Zheng. Random walks and isoperimetric profiles under moment conditions. preprint, 2014.

[28] Laurent Saloff-Coste and Tianyi Zheng. Random walks on free solvable groups. Math. Z., 279(3-4):811-848, 2015.

[29] Dan Segal. The finite images of finitely generated groups. Proceedings of the London Mathematical Society, 82(03):597-613, 2000.

[30] Said Sidki. Automorphisms of one-rooted trees: growth, circuit structure, and acyclicity. J. Math. Sci. (New York), 100(1):1925-1943, 2000. Algebra, 12.

[31] Romain Tessera. Isoperimetric profile and random walks on locally compact solvable groups. Rev. Mat. Iberoam., 29(2):715-737, 2013.

[32] John S. Wilson. Further groups that do not have uniformly exponential growth. J. Algebra, 279(1):292-301, 2004.

[33] John S. Wilson. On exponential growth and uniformly exponential growth for groups. Invent. Math., 155(2):287-303, 2004. 